Prepared in cooperation with the Washington County Water Conservancy District

\title{
Assessment of Managed Aquifer Recharge at Sand Hollow Reservoir, Washington County, Utah, Updated to Conditions Through 2016
}

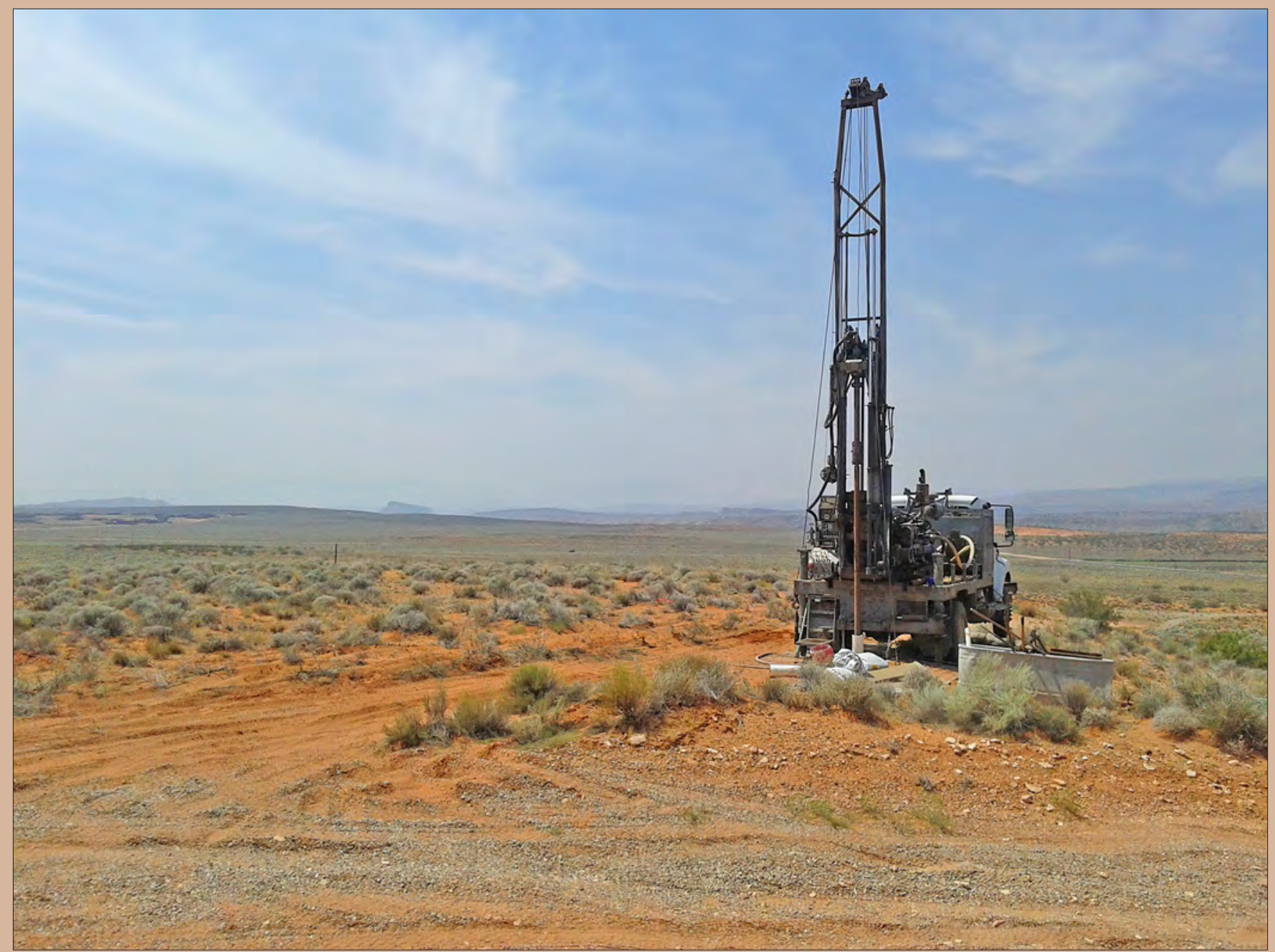

Open-File Report 2018-1140 
Cover photograph: View of drilling operations at well WD 22 near Sand Hollow Reservoir, Utah, looking west. Photograph by Tom M. Marston, U.S. Geological Survey, August 2015. 


\section{Assessment of Managed Aquifer Recharge at Sand Hollow Reservoir, Washington County, Utah, Updated to Conditions Through 2016}

By Thomas M. Marston and Nora C. Nelson

Prepared in cooperation with the Washington County Water Conservancy District

Open-File Report 2018-1140 


\title{
U.S. Department of the Interior \\ RYAN K. ZINKE, Secretary
}

\author{
U.S. Geological Survey \\ James F. Reilly II, Director
}

U.S. Geological Survey, Reston, Virginia: 2018

For more information on the USGS - the Federal source for science about the Earth, its natural and living resources, natural hazards, and the environment-visit https://www.usgs.gov or call 1-888-ASK-USGS.

For an overview of USGS information products, including maps, imagery, and publications,

visit https://store.usgs.gov.

Any use of trade, firm, or product names is for descriptive purposes only and does not imply endorsement by the U.S. Government.

Although this information product, for the most part, is in the public domain, it also may contain copyrighted materials as noted in the text. Permission to reproduce copyrighted items must be secured from the copyright owner.

Suggested citation:

Marston, T.M., and Nelson, N.C., 2018, Assessment of managed aquifer recharge at Sand Hollow Reservoir, Washington County, Utah, updated to conditions through 2016: U.S. Geological Survey Open-File Report 2018-1140, 38 p., https://doi.org/10.3133/ofr20181140. 


\section{Contents}

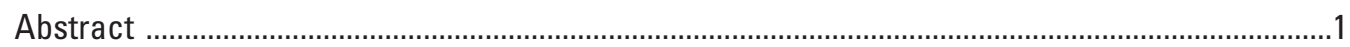

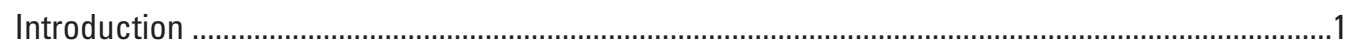

Assessment of Managed Aquifer Recharge at Sand Hollow Reservoir ..........................................

Data Collection Methods and Results .................................................................................

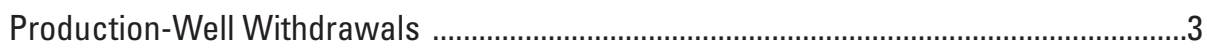

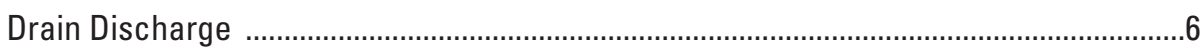

Groundwater-Level Data and Reservoir Altitude ...........................................................7

Surface-Water Inflow To and Outflow From Sand Hollow Reservoir ..............................8

Meteorological Data ....................................................................................................19

Estimates of Managed Aquifer Recharge From Sand Hollow Reservoir ................................20

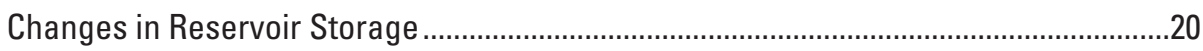

Reservoir Evaporation ....................................................................................................21

Estimates of Total Volume of Managed Aquifer Recharge From

Sand Hollow Reservoir ...................................................................................... 21

Groundwater and Surface-Water Quality in Sand Hollow ...........................................................2

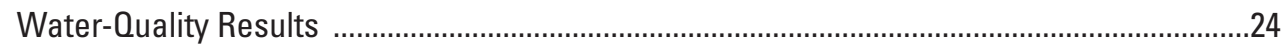

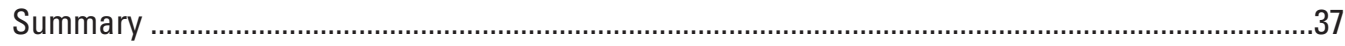

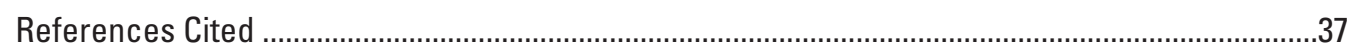

\section{Figures}

1. Map showing location of the Sand Hollow Reservoir study area,

Washington County, Utah

2. Map showing location of monitoring and production wells, weather stations, drains, and surface-water sampling sites in and near Sand Hollow Reservoir, Washington County, Utah

3. Graph showing ashington County Water Conservancy District production-well withdrawals in Sand Hollow, Washington County, Utah, 2004-16

4. Graph showing monthly reported discharge from the North and West Dam drains, and the West Dam Spring drain in relation to reservoir altitude at Sand Hollow Reservoir, Washington County, Utah, 2003-16

5. Graph showing water-level altitude in monitoring wells in relation to Sand Hollow Reservoir altitude, Sand Hollow, Washington County, Utah, 1995-2016

6. Map showing potentiometric surface of the Navajo aquifer in December 2016, at Sand Hollow Reservoir, Washington County, Utah

7. Graph showing monthly precipitation at Sand Hollow Reservoir, Washington County, Utah, 1998-2016

8. Graph showing monthly estimated evaporation and groundwater recharge in relation to reservoir altitude at Sand Hollow Reservoir, Washington County, Utah, $2002-16$

9. Graph showing monthly calculated groundwater recharge rates beneath Sand Hollow Reservoir, Washington County, Utah, 2002-16

10. Graph showing estimated annual net inflow, evaporation, and groundwater recharge at Sand Hollow Reservoir, Washington County, Utah, 2002-16 


\section{Tables}

1. Reservoir data, evaporation, precipitation, and calculated recharge beneath Sand Hollow Reservoir, Washington County, Utah, 2002-16.

2. Field measurements, total dissolved-gas pressure, and concentrations of dissolved organic carbon, tritium, chlorofluorocarbons, and sulfur hexafluoride in groundwater and surface water from Sand Hollow, Washington County, Utah..........25

3. Field measurements and concentrations of major ions, selected trace elements, and nutrients in groundwater and surface water from selected sites in Sand Hollow, Washington County, Utah 


\section{Conversion Factors, Datums, and Abbreviated Water-Quality Units}

U.S. customary units to International System of Units

\begin{tabular}{|c|c|c|}
\hline Multiply & By & To obtain \\
\hline \multicolumn{3}{|c|}{ Length } \\
\hline inch (in.) & 2.54 & centimeter $(\mathrm{cm})$ \\
\hline foot (ft) & 0.3048 & meter $(\mathrm{m})$ \\
\hline mile (mi) & 1.609 & kilometer $(\mathrm{km})$ \\
\hline \multicolumn{3}{|c|}{ Volume } \\
\hline acre foot (acre-ft) & $1,233.48$ & cubic meter (m) \\
\hline \multicolumn{3}{|c|}{ Hydraulic conductivity } \\
\hline foot per day (ft/d) & 0.3048 & meter per day $(\mathrm{m} / \mathrm{d})$ \\
\hline
\end{tabular}

Temperature in degrees Celsius $\left({ }^{\circ} \mathrm{C}\right)$ can be converted to degrees Fahrenheit $\left({ }^{\circ} \mathrm{F}\right)$ as follows:

${ }^{\circ} \mathrm{F}=\left(1.8 \mathrm{x}^{\circ} \mathrm{C}\right)+32$

Temperature in degrees Fahrenheit $\left({ }^{\circ} \mathrm{F}\right)$ can be converted to degrees Celsius $\left({ }^{\circ} \mathrm{C}\right)$ as follows:

${ }^{\circ} \mathrm{C}=\left({ }^{\circ} \mathrm{F}-32\right) / 1.8$

Horizontal coordinate information is referenced to the North American Datum of 1983 (NAD 83).

Vertical coordinate information is referenced to the North American Vertical Datum of 1988 (NAVD 88).

Altitude, as used in this report, refers to distance above the vertical datum.

Specific conductance is given in microsiemens per centimeter at 25 degrees Celsius $(\mu \mathrm{S} / \mathrm{cm}$ at $\left.25^{\circ} \mathrm{C}\right)$.

Total dissolved-gas pressure is reported in millimeters of mercury $(\mathrm{mm} \mathrm{Hg})$, where $760 \mathrm{~mm} \mathrm{Hg}$ equals one atmosphere.

Concentrations of chemical constituents in water are given either in milligrams per liter (mg/L) or micrograms per liter $(\mu \mathrm{g} / \mathrm{L})$.

Tritium units (TU) are used to report tritium concentration, where $1 \mathrm{TU}$ equals tritium concentration in picoCuries per liter divided by 3.22 .

Chlorofluorocarbon (CFC) concentrations are reported as picomoles per kilogram (pmol/kg).

Sulfur hexafluoride $\left(\mathrm{SF}_{6}\right)$ concentrations are reported as femtomoles per kilogram (fmol/kg). 


\section{Abbreviations}

$\begin{array}{ll}\text { CFCs } & \text { chlorofluorocarbons } \\ \text { CI/Br } & \text { chloride to bromide ratio } \\ \text { DO } & \text { dissolved oxygen } \\ \text { DOC } & \text { dissolved organic carbon } \\ \text { MAR } & \text { managed aquifer recharge } \\ \text { NRCS } & \text { Natural Resources Conservation Service } \\ \text { SCAN } & \text { Soil Climate Analysis Network } \\ \text { TDG } & \text { total dissolved gas } \\ \text { USGS } & \text { U.S. Geological Survey } \\ \text { WCWCD } & \text { Washington County Water Conservancy District } \\ \text { WD } & \text { water district }\end{array}$




\title{
Assessment of Managed Aquifer Recharge at Sand Hollow Reservoir, Washington County, Utah, Updated to Conditions Through 2016
}

\author{
By Thomas M. Marston and Nora C. Nelson
}

\section{Abstract}

Sand Hollow Reservoir in Washington County, Utah, was completed in March 2002 and is operated primarily for managed aquifer recharge by the Washington County Water Conservancy District. From 2002 through 2016, surfacewater diversions of about 256,000 acre-feet (acre-ft) to Sand Hollow Reservoir have allowed the reservoir to remain nearly full since 2006. Groundwater levels in monitoring wells near the reservoir rose through 2006 but have fluctuated more recently because of variations in reservoir stage and nearby pumping from production wells. Between 2004 and 2016, about 37,000 acre-ft of groundwater was withdrawn by these wells for municipal supply. In addition, about 37,000 acre- $\mathrm{ft}$ of shallow seepage was captured by French drains adjacent to the North and West Dams and used for municipal supply, irrigation, or returned to the reservoir. From 2002 through 2016, about 141,000 acre-ft of water seeped beneath the reservoir to recharge the underlying Navajo Sandstone aquifer, which includes about 14,200 acre-ft of recharge during the 2015-16 time period since the last report published in 2016.

Water quality continued to be monitored at various wells in Sand Hollow during 2015-16 to evaluate the timing and location of reservoir recharge as it moved through the aquifer. Changing geochemical conditions at monitoring wells WD 4 and WD 12 indicate rising groundwater levels and mobilization of vadose-zone salts, which could be a precursor to the arrival of reservoir recharge.

\section{Introduction}

Sand Hollow Reservoir (fig. 1) in Washington County, Utah, was completed in March 2002 and is operated primarily for managed aquifer recharge (MAR) by the Washington County Water Conservancy District (WCWCD). The reservoir is an off-channel facility that receives water diverted from the Virgin River near the town of Virgin, Utah. Sand Hollow has been the subject of interdisciplinary, cooperative investigations of groundwater hydrology and geochemistry since 1999. Previous Sand Hollow reports document pre-reservoir vadose-zone and groundwater conditions prior to March 2002 (Heilweil and Solomon, 2004; Heilweil and others, 2006; Heilweil and others, 2007; Heilweil and McKinney, 2007; Heilweil, Solomon, and Ortiz, 2009b), pond and trench infiltration studies adjacent to the reservoir (Heilweil and others, 2004; Heilweil and Watt, 2011), and post-reservoir groundwater conditions, water budgets, and estimates of groundwater recharge from the reservoir from March 2002 through December 2014 (Heilweil and others, 2005; Heilweil and Susong, 2007; Heilweil, Ortiz, and Susong, 2009; Heilweil and Marston, 2011; Marston and Heilweil, 2013; Marston and Heilweil, 2016). These reports also contain monitoring-well and production-well completion information, as well as historical water-quality and precipitation data.

The objectives of this report are to present and interpret (1) groundwater levels, reservoir altitude, well withdrawals, drain discharge, meteorological data, reservoir water temperature, and inflows/outflows from March 2002 through December 2016 for estimating monthly amounts of MAR from Sand Hollow Reservoir to the Navajo Sandstone, and (2) groundwater and surface-water chemical data collected prior to the construction of the reservoir through April 2016 for evaluating groundwater flow paths and travel times of this MAR. This study is a cooperative effort by the WCWCD and the U.S. Geological Survey (USGS). Funding for this work was provided by both the USGS and the WCWCD. 


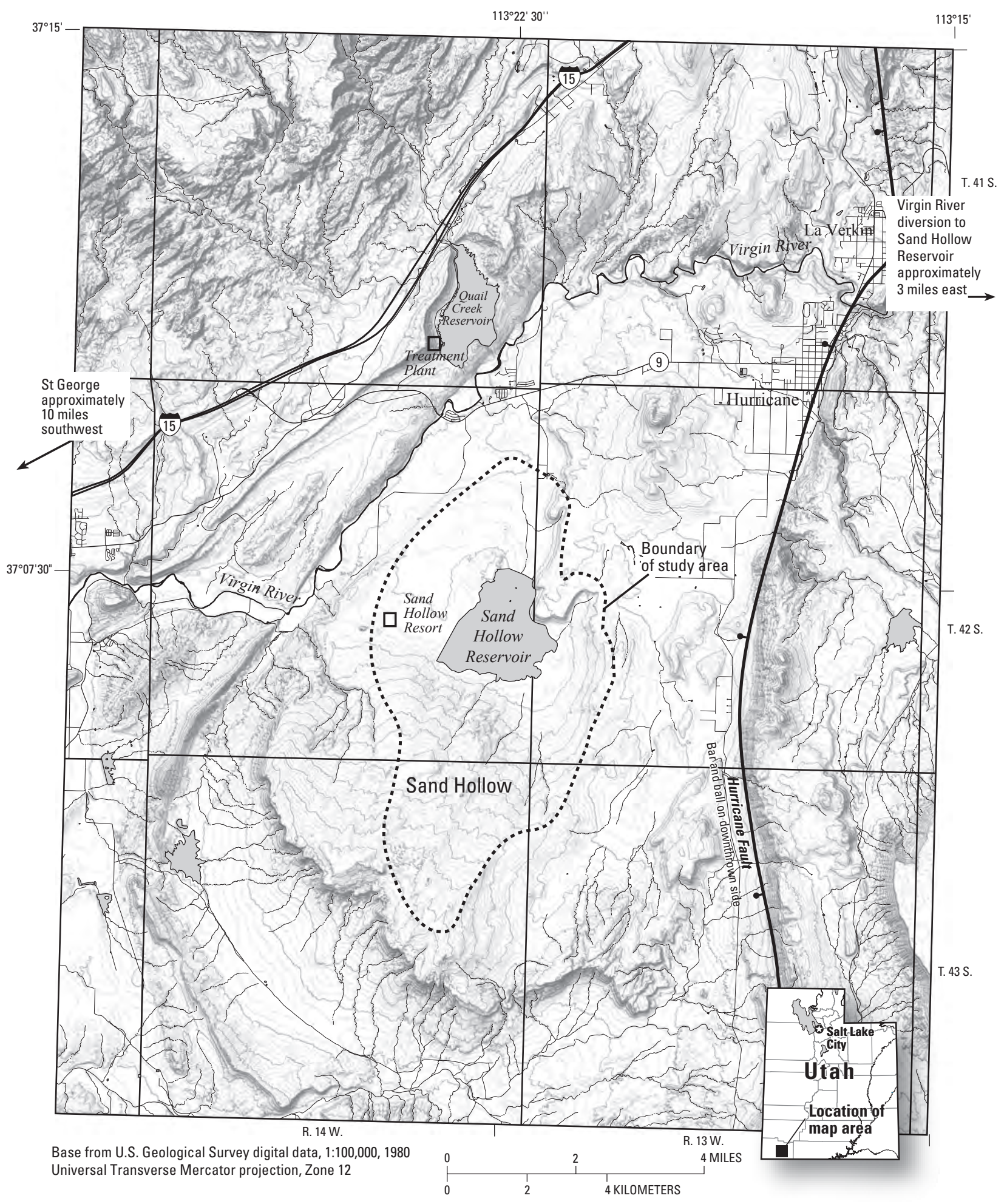

Figure 1. Location of the Sand Hollow Reservoir study area, Washington County, Utah. 


\section{Assessment of Managed Aquifer Recharge at Sand Hollow Reservoir}

Many different types of data have been collected to investigate recharge processes, quantify recharge from Sand Hollow Reservoir, and to evaluate hydraulic and geochemical changes in the underlying Navajo Sandstone aquifer (Navajo aquifer). These data include production-well withdrawals near the reservoir, amounts of pumpage from drains capturing shallow groundwater discharge adjacent to the reservoir, reservoir and monitoring-well water levels, inflows and outflows through the pipeline connecting Sand Hollow Reservoir with the Virgin River and Quail Creek Reservoir and treatment plant, meteorological parameters, and reservoir water temperatures (fig. 1). Water chemistry of groundwater and Sand Hollow Reservoir water was evaluated through field water-quality parameters (water temperature, specific conductance, $\mathrm{pH}$, dissolved oxygen (DO), and total dissolvedgas (TDG) pressure), and analysis of dissolved constituents including major ions and trace elements, dissolved organic carbon (DOC), tritium $\left({ }^{3} \mathrm{H}\right)$, and industrial dissolved gases.

\section{Data Collection Methods and Results}

Data collection methods are described in detail in Heilweil and others (2005) and briefly summarized in the following sections.

\section{Production-Well Withdrawals}

The WCWCD has 13 production wells completed in the Navajo Sandstone that are available to capture both preexisting groundwater (natural recharge) in Sand Hollow and recharge from Sand Hollow Reservoir (fig. 2). The WCWCD and other water users have withdrawn groundwater from natural recharge in Sand Hollow for many years. The WCWCD groundwater withdrawals are recorded monthly from inline magnetic flow meters installed at each well. Since August 2004, monthly withdrawals by the WCWCD have generally exceeded 150 acre-feet (acre-ft), except for several months during the winters of 2004-05, 2005-06, 2008-09, and 2010 (fig. 3). The majority of this pumping was from wells 8 and 9 through late 2012, both located adjacent to the North Dam. Starting in early 2013, some of the pumping has been shifted to wells 19, 21, and 23. From 2004 through 2006, there were minimal withdrawals from these wells during the winter. Since 2006, withdrawals have been more constant yearround. Monthly withdrawals from production wells averaged about 260 acre-ft from March 2006 through December 2016. Smaller amounts have been sporadically withdrawn from wells 1,2, and 17. Approximately 37,000 acre-ft were pumped from the WCWCD production wells from January 2004 through December 2016. Through 2016, withdrawals by the WCWCD at Sand Hollow have been permitted by the Utah Division of Water Rights as natural recharge in Sand Hollow. These withdrawals are governed by different water rights than water recharged from Sand Hollow Reservoir; withdrawal rights for this artificial recharge have not yet been exercised. 


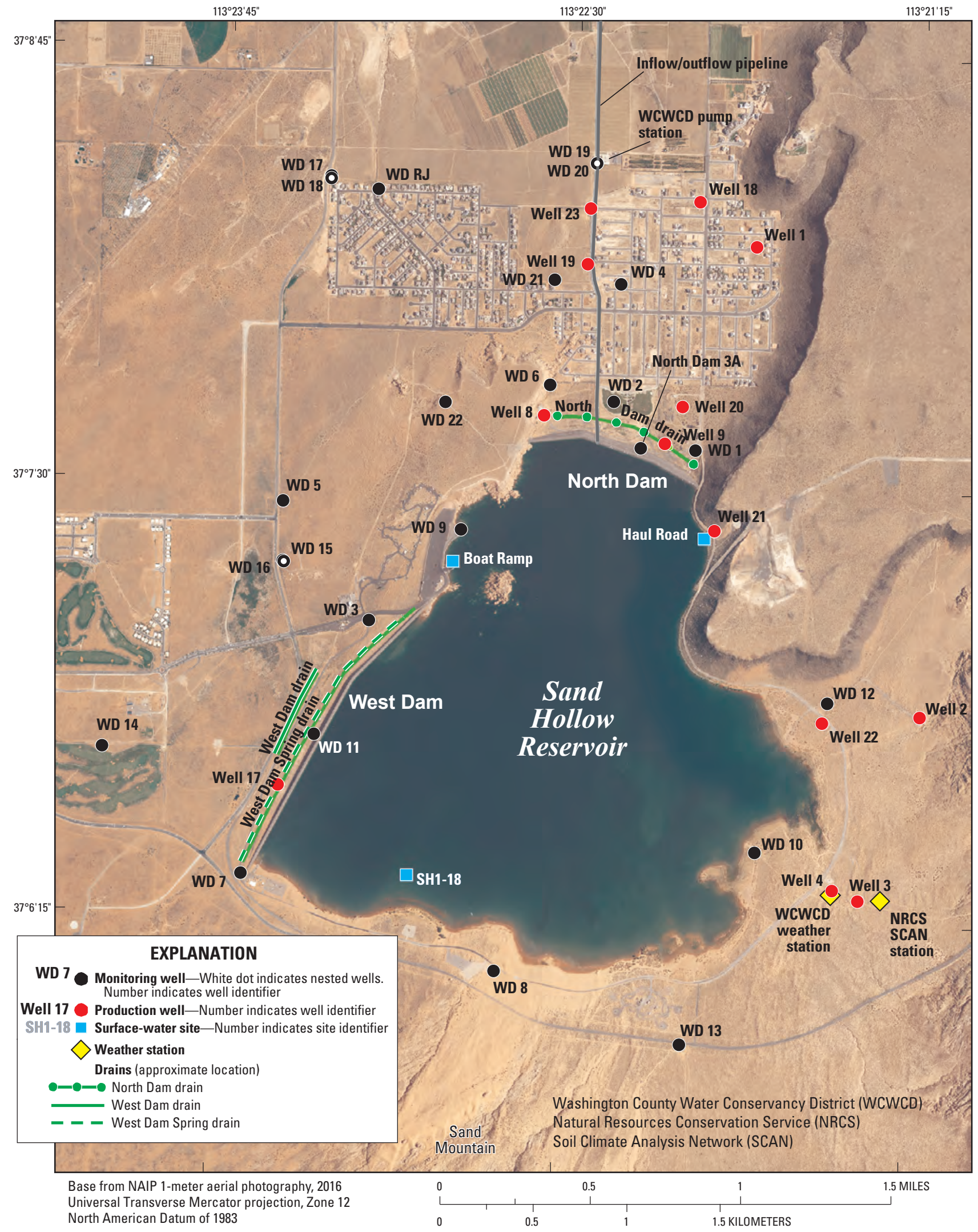

Figure 2. Location of monitoring and production wells, weather stations, drains, and surface-water sampling sites in and near Sand Hollow Reservoir, Washington County, Utah. 


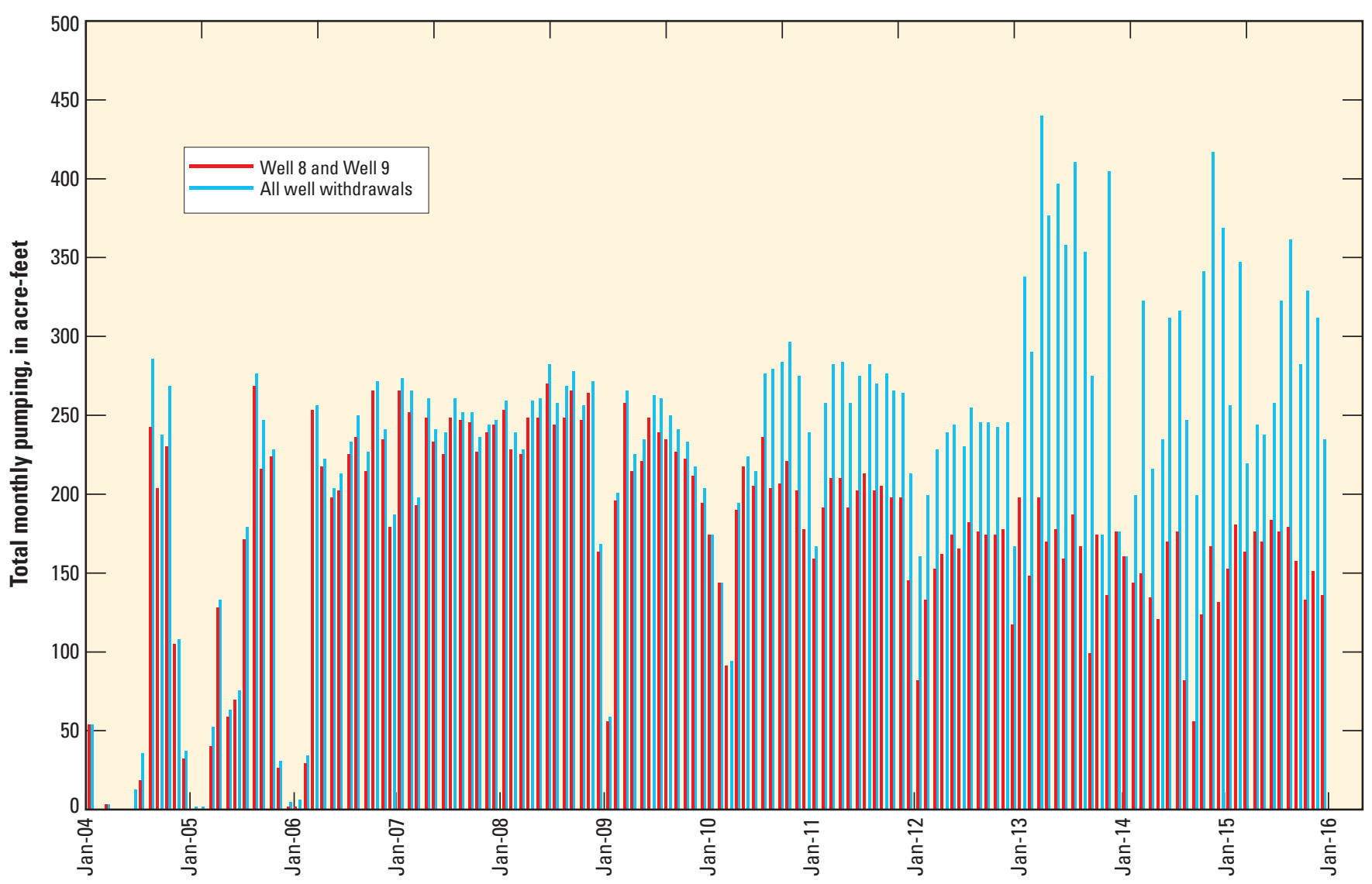

Figure 3. Washington County Water Conservancy District production-well withdrawals in Sand Hollow, Washington County, Utah, 2004-16. 


\section{Drain Discharge}

Because of the steep hydraulic gradients associated with the hydraulic connection between the reservoir and the underlying Navajo aquifer, some land-surface areas downgradient of the North and West Dams became saturated following construction of the reservoir. In response, three French drains (North Dam drain, West Dam drain, and West Dam Spring drain) were constructed for capturing this shallow groundwater (fig. 2). Timing of excavation and spatial dimensions for the three drains are described in Heilweil and Marston (2011).

The water pumped from drains is measured with a Tigermag totalizing flow meter (Sparling Instruments, El Monte, California). Discharge from the North Dam drain has been pumped at a relatively consistent rate (about 50 acre- $\mathrm{ft}$ per month) since January 2008 (fig. 4); about 9,000 acre-ft were pumped from the North Dam drain between 2003 and 2016. Initially, all of this water was returned to the reservoir, but since 2007, the majority of the water has been used by Sand Hollow Resort (fig. 1) to meet irrigation demands. About 2,300 acre-ft of water were pumped from the West Dam drain back into the reservoir from 2005 through 2016. Beginning in October 2006, pumping of discharge from the West Dam Spring drain was initiated and has largely replaced the need for pumping of the West Dam drain (fig. 1). From 2006 through 2016, about 26,000 acre-ft were pumped from the West Dam Spring drain for municipal use or returned back to Sand Hollow Reservoir. Although groundwater discharge to the West Dam Spring drain likely does not vary greatly, pumping from this drain has been intermittent. This intermittent pumping schedule results in high variability in the monthly reported discharge from the West Dam Spring drain (fig. 4).

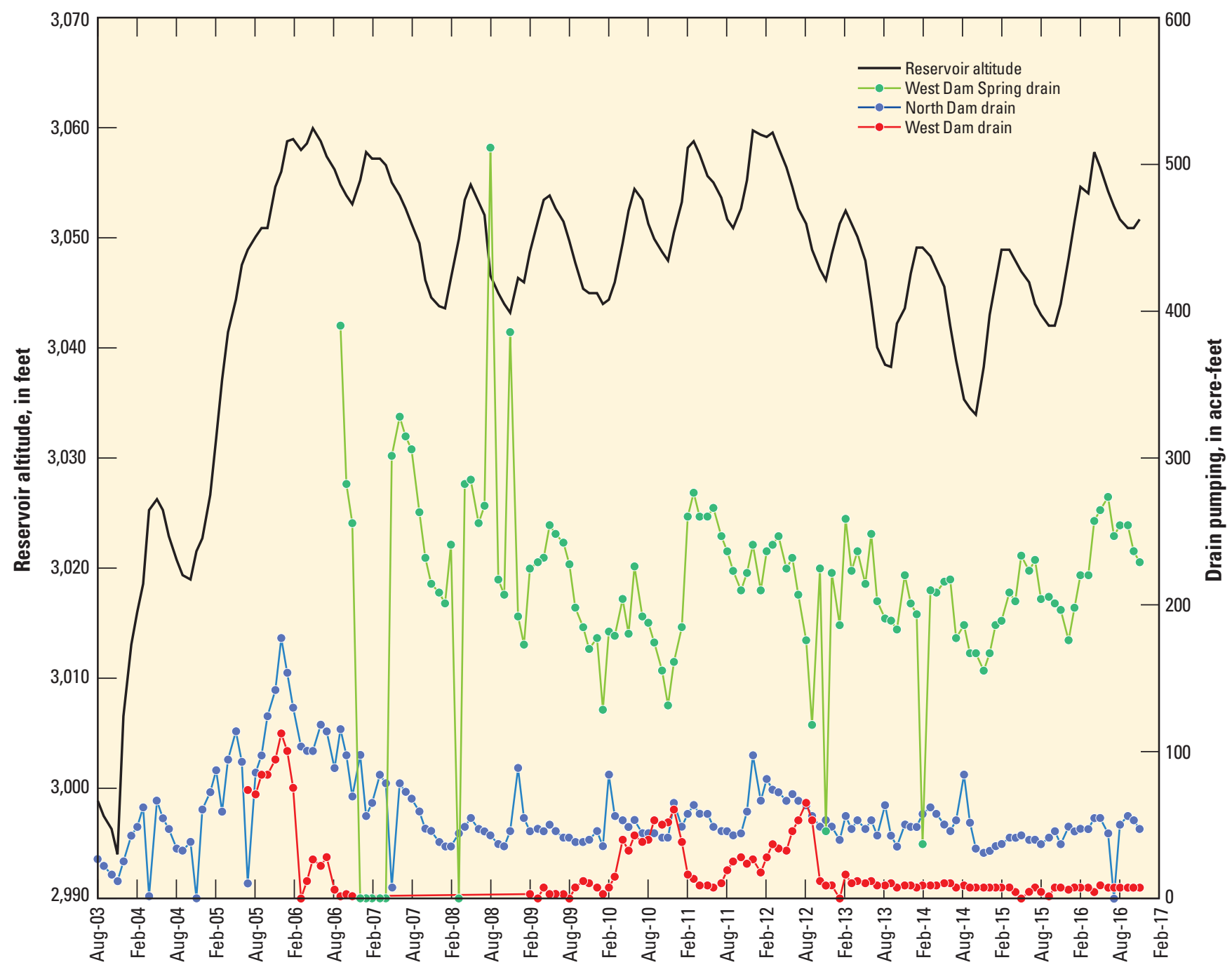

Figure 4. Monthly reported discharge from the North and West Dam drains, and the West Dam Spring drain in relation to reservoir altitude at Sand Hollow Reservoir, Washington County, Utah, 2003-16. 


\section{Groundwater-Level Data and Reservoir Altitude}

Groundwater levels measured in an extensive monitoringwell network surrounding Sand Hollow Reservoir were used to document changes in the potentiometric surface associated with recharge from Sand Hollow Reservoir. The WCWCD measures water levels monthly in 21 monitoring wells completed in the Navajo Sandstone (fig. 2; the WD RJ monitoring well was removed in 2013). These wells were constructed with either 1- or 2-inch-diameter PVC casing, with perforations along the bottom 5- to 20-foot (ft) length of the casing (Heilweil and others, 2005). Three locations have nested pairs of water district (WD) monitoring wells: WD 15 and WD 16, WD 17 and WD 18, and WD 19 and WD 20. The vertical distances between well screens for the nested-pair wells are $243 \mathrm{ft}, 79 \mathrm{ft}$, and $227 \mathrm{ft}$, respectively. Water levels were measured by the WCWCD using electric-tape waterlevel indicators. Annual independent check measurements were done by USGS personnel for quality assurance to ensure accuracy (repeatability) of the instruments.
Daily reservoir water-level altitude (stage) was recorded from August 2003 through December 2016 by using a pressure transducer installed by the WCWCD in the reservoir along the North Dam. Because of periods of transducer malfunction from 2005 through 2007, and from August 2011 to December 2011, daily reservoir stage was interpolated on the basis of monthly measurements recorded at the boat ramp by WCWCD and Sand Hollow State Park personnel, and then correlated with trends from the transducer data.

Recently measured (January 2014 through December 2016) and previously reported (Heilweil and others, 2005; Heilweil and Susong, 2007; Heilweil, Ortiz, and Susong, 2009; Heilweil and Marston, 2011; Marston and Heilweil, 2013; Marston and Heilweil, 2016) groundwater levels and reservoir water-level altitudes are shown on figure 5. The reservoir stage rose from about $2,980 \mathrm{ft}$ at the beginning of March 2002 to a maximum of about 3,060 ft in May 2006, when the reservoir was first filled to capacity. The reservoir altitude receded to about 3,040 ft in December 2007 as a result of reduced inflows and evaporative losses, and then fluctuated

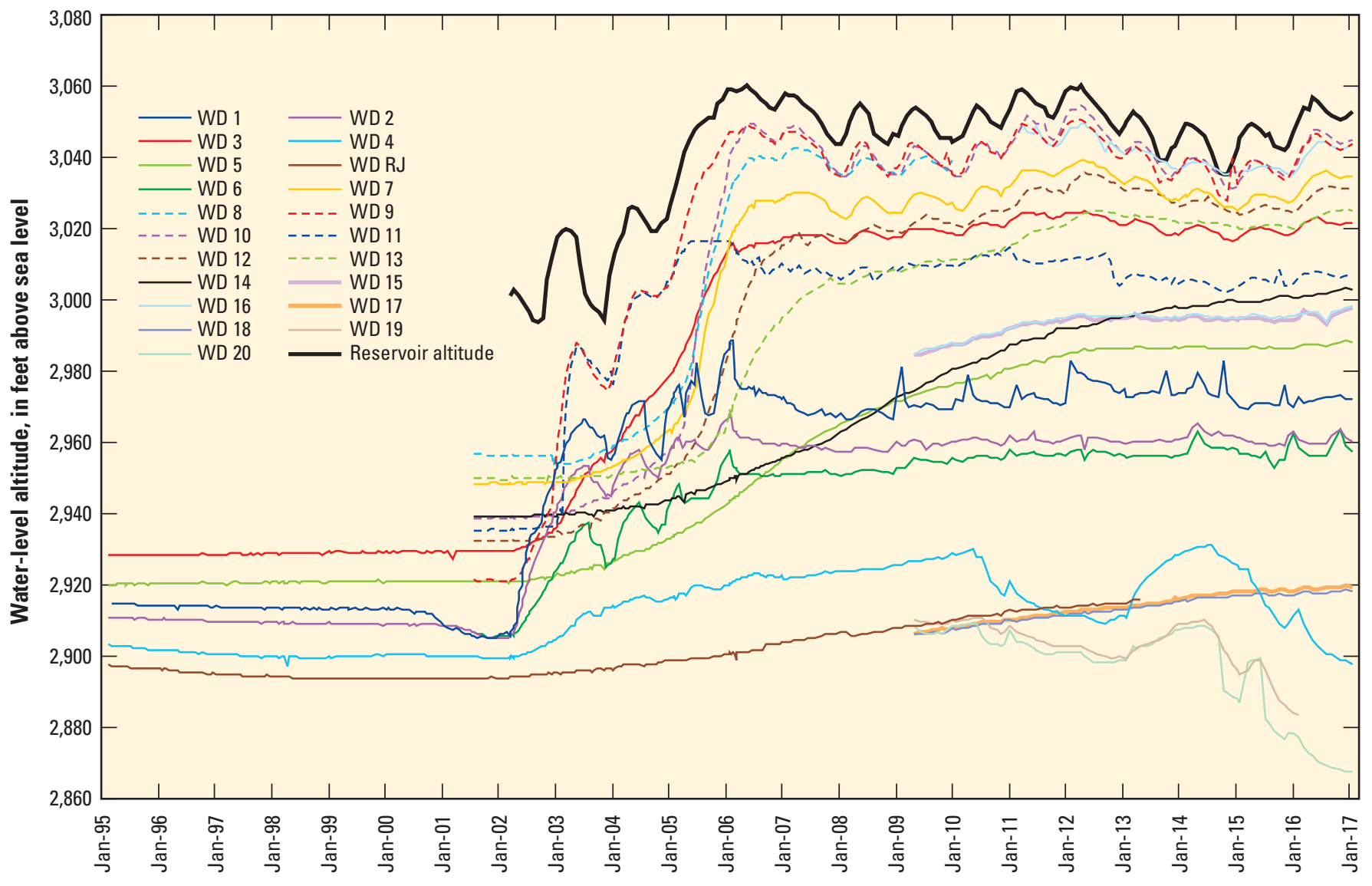

Figure 5. Water-level altitude in monitoring wells in relation to Sand Hollow Reservoir altitude, Sand Hollow, Washington County, Utah, 1995-2016. 
between about 3,040 and 3,060 ft from 2008 through 2011. From 2012 through 2014, the reservoir altitude dropped to a fluctuating level between about 3,030 and 3,050 ft. From 2014 to 2016 , the reservoir altitude rose again to a fluctuating level between about 3,040 and 3,055 ft.

On the basis of water-level measurements in the 20 monitoring wells, altitude of the water table (or potentiometric surface) near Sand Hollow Reservoir during December 2016 ranged from 2,868 to $3,044 \mathrm{ft}$ (fig. 6). The reservoir altitude during this same period was about 3,050 ft. The lines on figure 6 represent estimated potentiometric contours in the aquifer, which indicate lines of equal groundwater-level altitude, and the arrows indicate generalized directions of horizontal groundwater movement away from the reservoir. Horizontal hydraulic gradients, calculated by dividing the difference in water-level altitude between two points by the distance separating these locations, indicate the potential horizontal direction of groundwater movement. The steepest horizontal hydraulic gradients are beneath the North and West Dams and generally decline with increasing distance from the reservoir. The steep hydraulic gradients beneath the dams are the combined result of low-conductivity materials at the core of the dams and pumping in wells and drains at the base of both dams. For example, the horizontal hydraulic gradient between Sand Hollow Reservoir (reservoir altitude of 3,050 ft) and WD 1 (groundwater altitude of 2,972 ft) in 2016 was 0.136 foot per foot $(\mathrm{ft} / \mathrm{ft})$, whereas the gradient between WD 6 (groundwater altitude of 2,960 ft) and WD 19 (2,898 ft) was $0.015 \mathrm{ft} / \mathrm{ft}$. In 2016, due to MAR, the resulting regional hydraulic gradient between WD 9 (groundwater altitude of 3,042 ft) and WD $17(2,920 \mathrm{ft})$ was $0.019 \mathrm{ft} / \mathrm{ft}$.

Pumping rates increased at wells 19 and 23 starting in early 2013. Increased withdrawals have induced a drawdown cone around these production wells north of the reservoir; the drawdowns are apparent in nearby monitoring wells WD 4, WD 19, and WD 20 (fig. 5). Well WD 19 went dry in March 2016 as a result of the local water table falling below the depth of the screened interval.

\section{Surface-Water Inflow To and Outflow From Sand Hollow Reservoir}

Surface water is pumped into and flows out of Sand Hollow Reservoir through a 60 -inch-diameter pipeline that enters through an inlet structure at the North Dam (fig. 2). This pipeline is part of a network of pipelines that connect the Virgin River, Sand Hollow Reservoir, Quail Creek Reservoir, and the Quail Creek Reservoir Water Treatment Facility. The WCWCD has the capability to move water within this network of pipelines by using gravity-induced flow and inline pumping. Sand Hollow Reservoir is currently managed to maximize groundwater recharge, and little water has been removed from the reservoir. Monthly surface-water inflow to and outflow from Sand Hollow Reservoir is shown in table 1. The "Monthly pump station inflow or outflow" column in this table is the amount of Sand Hollow Reservoir water coming in from the Virgin River or going out to Quail Creek Reservoir, the Quail Creek Water Treatment Plant, or other facilities (fig. 1). These data were collected at the WCWCD pump station about 1 mile north of the North Dam. Five pumps, each with Sparling Tigermag totalizing flow meters, are linked to a computer system that combines and records total daily discharge in gallons. The flow meters have electronic modules on which calibration diagnostics are done monthly by the WCWCD. Each module is removed annually for factory recalibration.

The "Monthly drain and spring return flow to reservoir" column in table 1 is the portion of discharge to the three drains that is pumped back into Sand Hollow Reservoir. The "Monthly outflow to Sand Hollow Resort" column is the amount of water required by the resort that cannot be met by discharge to the North Dam drain and is fulfilled by outflow of stored water from Sand Hollow Reservoir. Therefore, the "Monthly total inflow or outflow to/from reservoir" column is a sum of the pump station inflow/outflow, the drain and spring return flow, and the outflow to Sand Hollow Resort (table 1).

The "Monthly pump station inflow or outflow" column is unchanged from Heilweil and Marston (2011) and is comparable to the "Total surface-water inflow or outflow" column in table 7 of Heilweil and others (2005) from March 2002 to August 2004; the "Monthly surface-water inflow or outflow" column in table 2 of Heilweil and Susong (2007) from September 2004 to August 2006; and the "Monthly net surface-water inflow/outflow" column in table 2 of Heilweil, Ortiz, and Susong (2009) from September 2006 to December 2007.

As in Heilweil and Marston (2011), both "Monthly drain and spring return flow to reservoir" and "Monthly outflow to Sand Hollow Resort" are included in calculations of total inflow to and outflow from the reservoir. These amounts are added to the "Monthly pump station inflow or outflow" column and summed in the "Monthly total inflow or outflow to/from reservoir" column. Monthly total inflow/outflow amounts from March 2002 through December 2016 ranged from about $-5,200$ acre-ft to 6,600 acre-ft. Approximately 256,000 acre-ft of total net inflow were pumped into Sand Hollow Reservoir from the Virgin River from 2002 through 2016. 


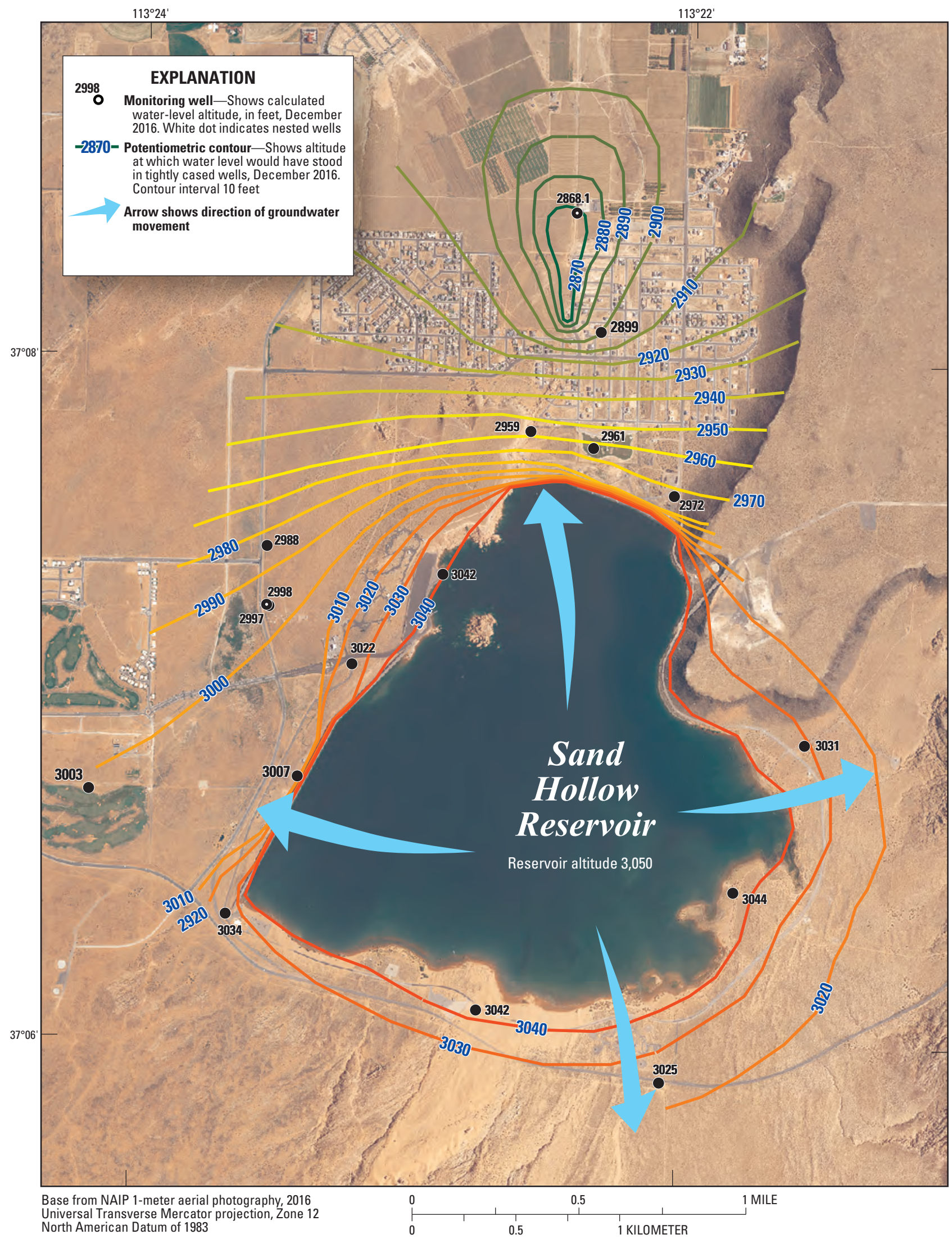

Figure 6. Potentiometric surface of the Navajo aquifer in December 2016, at Sand Hollow Reservoir, Washington County, Utah. 
Table 1. Reservoir data, evaporation, precipitation, and calculated recharge beneath Sand Hollow Reservoir, Washington County, Utah, 2002-16.

[Reservoir altitude and Reservoir storage: value is from the last day of each month; Reservoir surface area: value is an average of the daily values for each month; $2 o, 2$ standard deviation; $\mathrm{ft} / \mathrm{d}$, foot per day; —, no data available]

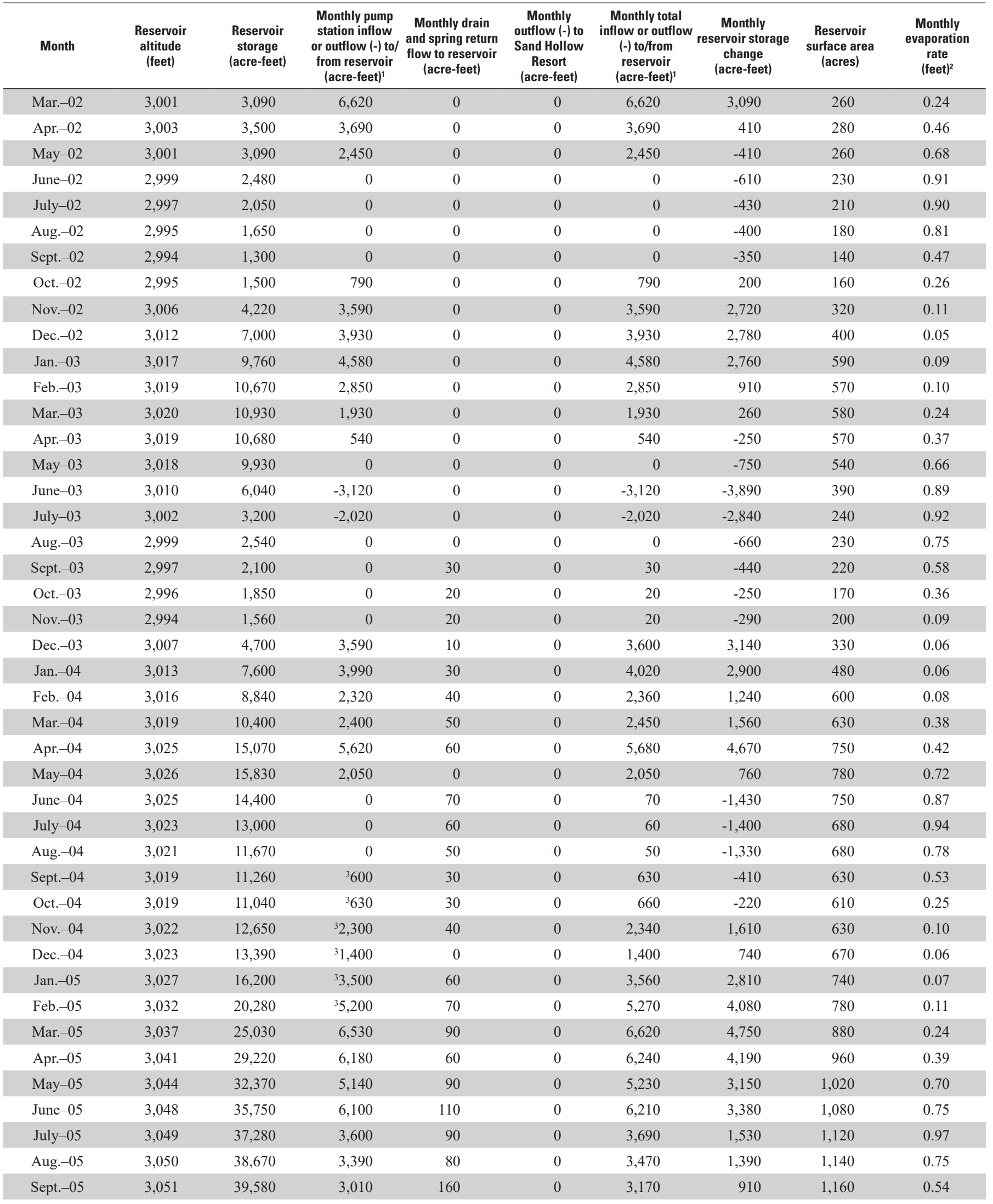


Table 1. Reservoir data, evaporation, precipitation, and calculated recharge beneath Sand Hollow Reservoir, Washington County, Utah, 2002-16.-Continued

[Reservoir altitude and Reservoir storage: value is from the last day of each month; Reservoir surface area: value is an average of the daily values for each month; 20,2 standard deviation; ft/d, foot per day; - , no data available]

\begin{tabular}{|c|c|c|c|c|c|c|}
\hline Month & $\begin{array}{c}\text { Monthly } \\
\text { evaporation } \\
\text { (acre-feet) }\end{array}$ & $\begin{array}{c}\text { Monthly } \\
\text { precipitation } \\
\text { (acre-feet) }\end{array}$ & $\begin{array}{l}\text { Monthly } \\
\text { groundwater } \\
\text { recharge } \\
\text { (acre-feet) }\end{array}$ & $\begin{array}{l}\text { Monthly } \\
\text { groundwater } \\
\text { recharge } \\
\text { uncertainty, 2 } \sigma \\
\text { (percent) }\end{array}$ & $\begin{array}{l}\text { Monthly } \\
\text { groundwater } \\
\text { recharge } \\
\text { uncertainty, } 2 \sigma \\
\text { (acre-feet) }\end{array}$ & $\begin{array}{c}\text { Groundwater } \\
\text { recharge rate } \\
\text { (ft/d) }\end{array}$ \\
\hline Mar.-02 & 60 & - & 3,470 & 6.7 & 232 & 0.430 \\
\hline Apr.-02 & 130 & - & 3,150 & 5.9 & 187 & 0.383 \\
\hline Мay-02 & 180 & - & 2,680 & 6.6 & 176 & 0.330 \\
\hline June-02 & 210 & - & 400 & 12.6 & 50 & 0.058 \\
\hline July-02 & 190 & - & 240 & 13.1 & 31 & 0.040 \\
\hline Aug.-02 & 150 & - & 250 & 12.7 & 32 & 0.044 \\
\hline Sept.-02 & 70 & - & 280 & 11.7 & 33 & 0.070 \\
\hline Oct.- 02 & 40 & - & 550 & 6.6 & 36 & 0.110 \\
\hline Nov.-02 & 30 & - & 840 & 7.2 & 61 & 0.090 \\
\hline Dec. -02 & 20 & - & 1,130 & 7.1 & 80 & 0.090 \\
\hline Jan.-03 & 50 & - & 1,770 & 7.0 & 123 & 0.097 \\
\hline Feb. -03 & 60 & - & 1,880 & 6.4 & 121 & 0.118 \\
\hline Mar.-03 & 140 & - & 1,530 & 6.5 & 99 & 0.085 \\
\hline Apr.-03 & 210 & - & 580 & 9.4 & 55 & 0.034 \\
\hline Мay-03 & 360 & - & 390 & 13.2 & 52 & 0.023 \\
\hline June-03 & 350 & - & 420 & 8.4 & 35 & 0.036 \\
\hline July-03 & 220 & - & 600 & 8.4 & 51 & 0.081 \\
\hline Aug.-03 & 170 & - & 490 & 12.0 & 59 & 0.069 \\
\hline Sept.-03 & 130 & - & 340 & 12.3 & 42 & 0.052 \\
\hline Oct.-03 & 60 & - & 210 & 11.9 & 25 & 0.040 \\
\hline Nov.-03 & 20 & - & 290 & 10.6 & 31 & 0.048 \\
\hline Dec. -03 & 20 & - & 440 & 7.4 & 32 & 0.043 \\
\hline Jan.-04 & 30 & - & 1,090 & 7.2 & 78 & 0.073 \\
\hline Feb.-04 & 40 & - & 1,080 & 6.9 & 74 & 0.064 \\
\hline Mar.-04 & 240 & - & 650 & 7.7 & 50 & 0.033 \\
\hline Apr.-04 & 310 & - & 700 & 7.6 & 53 & 0.031 \\
\hline May-04 & 560 & - & 730 & 8.6 & 63 & 0.030 \\
\hline June-04 & 650 & - & 850 & 13.1 & 112 & 0.038 \\
\hline July-04 & 640 & - & 820 & 13.1 & 108 & 0.039 \\
\hline Aug.-04 & 530 & - & 850 & 12.8 & 109 & 0.040 \\
\hline Sept.-04 & 330 & - & 710 & 10.2 & 73 & 0.038 \\
\hline Oct.- -04 & 150 & - & 730 & 8.4 & 61 & 0.039 \\
\hline Nov.-04 & 70 & - & 660 & 7.3 & 48 & 0.035 \\
\hline Dec.-04 & 40 & - & 620 & 7.0 & 43 & 0.030 \\
\hline Jan. -05 & 50 & - & 700 & 7.3 & 51 & 0.031 \\
\hline Feb. -05 & 80 & 130 & 1,240 & 7.3 & 91 & 0.057 \\
\hline Mar.-05 & 210 & 100 & 1,760 & 7.4 & 130 & 0.065 \\
\hline Apr.-05 & 370 & 130 & 1,810 & 7.5 & 136 & 0.063 \\
\hline May-05 & 710 & 40 & 1,410 & 7.9 & 112 & 0.045 \\
\hline June-05 & 810 & 20 & 2,040 & 7.8 & 160 & 0.063 \\
\hline July-05 & 1,080 & 10 & 1,090 & 8.8 & 96 & 0.031 \\
\hline Aug.--05 & 850 & 40 & 1,270 & 8.5 & 108 & 0.036 \\
\hline Sept. -05 & 630 & 20 & 1,650 & 8.1 & 133 & 0.047 \\
\hline
\end{tabular}




\section{Assessment of Managed Aquifer Recharge at Sand Hollow Reservoir, Washington County, Utah}

Table 1. Reservoir data, evaporation, precipitation, and calculated recharge beneath Sand Hollow Reservoir, Washington County, Utah, 2002-16.-Continued

[Reservoir altitude and Reservoir storage: value is from the last day of each month; Reservoir surface area: value is an average of the daily values for each month; $2 o, 2$ standard deviation; $\mathrm{ft} / \mathrm{d}$, foot per day; —, no data available]

\begin{tabular}{|c|c|c|c|c|c|c|c|c|c|}
\hline Month & $\begin{array}{l}\text { Reservoir } \\
\text { altitude } \\
\text { (feet) }\end{array}$ & $\begin{array}{l}\text { Reservoir } \\
\text { storage } \\
\text { (acre-feet) }\end{array}$ & $\begin{array}{l}\text { Monthly pump } \\
\text { station inflow } \\
\text { or outflow (-) to/ } \\
\text { from reservoir } \\
\text { (acre-feet) }\end{array}$ & $\begin{array}{l}\text { Monthly drain } \\
\text { and spring return } \\
\text { flow to reservoir } \\
\text { (acre-feet) }\end{array}$ & $\begin{array}{c}\text { Monthly } \\
\text { outflow (-) to } \\
\text { Sand Hollow } \\
\text { Resort } \\
\text { (acre-feet) }\end{array}$ & $\begin{array}{l}\text { Monthly total } \\
\text { inflow or outflow } \\
\text { (-) to/from } \\
\text { reservoir } \\
\text { (acre-feet) }^{1}\end{array}$ & $\begin{array}{l}\text { Monthly } \\
\text { reservoir storage } \\
\text { change } \\
\text { (acre-feet) }\end{array}$ & $\begin{array}{l}\text { Reservoir } \\
\text { surface area } \\
\text { (acres) }\end{array}$ & $\begin{array}{c}\text { Monthly } \\
\text { evaporation } \\
\text { rate } \\
(\text { feet })^{2}\end{array}$ \\
\hline Nov. -05 & 3,055 & 44,310 & 5,160 & 210 & 0 & 5,370 & ${ }^{4} 3,350$ & 1,230 & 0.11 \\
\hline Dec. -05 & 3,056 & 46,120 & 3,380 & 240 & 0 & 3,620 & 1,810 & 1,250 & 0.05 \\
\hline Mar.-06 & 3,058 & 48,700 & 60 & 210 & 0 & 270 & $-1,140$ & 1,310 & 0.18 \\
\hline Apr.-06 & 3,059 & 49,450 & 2,060 & 100 & 0 & 2,160 & 750 & 1,300 & 0.45 \\
\hline Мay-06 & 3,060 & 51,280 & 3,650 & 110 & 0 & 3,760 & 1,830 & 1,320 & 0.76 \\
\hline June-06 & 3,059 & 49,520 & 10 & 130 & 0 & 140 & $-1,760$ & 1,330 & 0.92 \\
\hline Oct. -06 & 3,054 & 43,390 & 30 & 120 & 0 & 150 & $-1,220$ & 1,230 & 0.22 \\
\hline Nov.-06 & 3,053 & 42,360 & 0 & 100 & 0 & 100 & $-1,030$ & 1,220 & 0.07 \\
\hline Dec.-06 & 3,055 & 45,100 & 4,430 & 70 & 0 & 4,500 & 2,740 & 1,230 & 0.04 \\
\hline Jan.-07 & 3,058 & 48,230 & 4,190 & 100 & 0 & 4,290 & 3,130 & 1,270 & 0.05 \\
\hline Feb.-07 & 3,057 & 47,630 & 30 & 60 & 0 & 90 & -600 & 1,290 & 0.13 \\
\hline Mar.-07 & 3,057 & 47,660 & 1,210 & 70 & 0 & 1,280 & 30 & 1,290 & 0.33 \\
\hline Apr.-07 & 3,057 & 46,720 & 50 & 80 & 0 & 130 & -940 & 1,280 & 0.45 \\
\hline Мay-07 & 3,055 & 44,880 & 0 & 0 & -110 & -110 & $-1,840$ & 1,220 & 0.74 \\
\hline June- 07 & 3,054 & 43,390 & 0 & 0 & -220 & -220 & $-1,490$ & 1,240 & 0.93 \\
\hline Jan.-08 & 3,044 & 31,470 & 0 & 20 & 0 & 20 & -210 & 1,030 & 0.06 \\
\hline Feb.-08 & 3,046 & 34,490 & 3,240 & 20 & 0 & 3,260 & 3,020 & 1,050 & 0.13 \\
\hline Mar.-08 & 3,050 & 38,460 & 4,420 & 0 & -70 & 4,350 & 3,970 & 1,110 & 0.29 \\
\hline Apr.-08 & 3,053 & 42,670 & 4,950 & 0 & -160 & 4,790 & 4,210 & 1,180 & 0.45 \\
\hline May-08 & 3,055 & 44,410 & 3,260 & 0 & -120 & 3,140 & 1,740 & 1,230 & 0.61 \\
\hline June- -08 & 3,053 & 42,540 & 0 & 0 & -220 & -220 & $-1,870$ & 1,230 & 0.93 \\
\hline July-08 & 3,052 & 41,080 & 0 & 0 & -180 & -180 & $-1,460$ & 1,180 & 0.95 \\
\hline Aug. -08 & 3,047 & 34,600 & ${ }^{7}-5,000$ & 0 & -180 & $-5,180$ & $-6,480$ & 1,140 & 0.82 \\
\hline Sept. -08 & 3,045 & 32,960 & 0 & 0 & -140 & -140 & $-1,640$ & 1,070 & 0.61 \\
\hline Oct. -08 & 3,044 & 31,890 & 0 & 0 & -70 & -70 & $-1,070$ & 1,050 & 0.36 \\
\hline Nov. -08 & 3,043 & 31,160 & 0 & 0 & -10 & -10 & -730 & 1,040 & 0.16 \\
\hline Dec. -08 & 3,046 & 34,490 & 4,100 & 40 & 0 & 4,140 & 3,330 & 1,050 & 0.06 \\
\hline Jan.-09 & 3,046 & 33,830 & 0 & 70 & 0 & 70 & -660 & 1,080 & 0.09 \\
\hline Feb.-09 & 3,049 & 37,770 & 4,630 & 50 & 0 & 4,680 & 3,940 & 1,110 & 0.14 \\
\hline Mar.-09 & 3,052 & 41,320 & 4,800 & 0 & -30 & 4,770 & 3,550 & 1,190 & 0.30 \\
\hline Apr.-09 & 3,055 & 44,030 & 3,920 & 0 & -70 & 3,850 & 2,710 & 1,220 & 0.44 \\
\hline
\end{tabular}


Table 1. Reservoir data, evaporation, precipitation, and calculated recharge beneath Sand Hollow Reservoir, Washington County, Utah, 2002-16.-Continued

[Reservoir altitude and Reservoir storage: value is from the last day of each month; Reservoir surface area: value is an average of the daily values for each month; $2 \alpha, 2$ standard deviation; ft/d, foot per day; -, no data available]

\begin{tabular}{|c|c|c|c|c|c|c|}
\hline Month & $\begin{array}{c}\text { Monthly } \\
\text { evaporation } \\
\text { (acre-feet) }\end{array}$ & $\begin{array}{c}\text { Monthly } \\
\text { precipitation } \\
\text { (acre-feet) }\end{array}$ & $\begin{array}{l}\text { Monthly } \\
\text { groundwater } \\
\text { recharge } \\
\text { (acre-feet) }\end{array}$ & $\begin{array}{l}\text { Monthly } \\
\text { groundwater } \\
\text { recharge } \\
\text { uncertainty, 2 } \sigma \\
\text { (percent) }\end{array}$ & $\begin{array}{l}\text { Monthly } \\
\text { groundwater } \\
\text { recharge } \\
\text { uncertainty, 2 } \sigma \\
\text { (acre-feet) }\end{array}$ & $\begin{array}{l}\text { Groundwater } \\
\text { recharge rate } \\
\text { (ft/d) }\end{array}$ \\
\hline Oct. -05 & ${ }^{4} 330$ & 60 & 1,490 & 7.6 & 113 & 0.040 \\
\hline Nov. -05 & 140 & 40 & 1,920 & 7.2 & 138 & 0.052 \\
\hline Dec. -05 & 60 & 20 & 1,770 & 6.9 & 122 & 0.046 \\
\hline Jan.-06 & 100 & 10 & 1,390 & 7.3 & 101 & 0.035 \\
\hline Feb. -06 & 160 & 30 & 1,070 & 7.3 & 78 & 0.029 \\
\hline Mar.-06 & 240 & 60 & 1,230 & 11.4 & 140 & 0.030 \\
\hline Apr.-06 & 580 & 40 & 870 & 8.7 & 76 & 0.022 \\
\hline May-06 & 1,000 & 0 & 930 & 8.7 & 81 & 0.023 \\
\hline June-06 & 1,220 & 10 & 690 & 14.1 & 97 & 0.017 \\
\hline July-06 & 1,160 & 30 & 640 & 14.1 & 90 & 0.016 \\
\hline Aug.-06 & 1,020 & 0 & 820 & 13.8 & 113 & 0.021 \\
\hline Sept.-06 & 650 & 10 & 1,070 & 12.8 & 137 & 0.028 \\
\hline Oct. -06 & 270 & 30 & 1,130 & 11.6 & 132 & 0.030 \\
\hline Nov.-06 & 90 & 0 & 1,040 & 10.8 & 112 & 0.028 \\
\hline Dec. -06 & 60 & 10 & 1,710 & 7.0 & 120 & 0.045 \\
\hline Jan. -07 & 60 & 10 & 1,110 & 7.2 & 80 & 0.028 \\
\hline Feb.-07 & 170 & 30 & 550 & 11.9 & 65 & 0.015 \\
\hline Mar.-07 & 430 & 0 & 820 & 9.0 & 73 & 0.021 \\
\hline Apr.-07 & 580 & 50 & 540 & 13.4 & 73 & 0.014 \\
\hline May-07 & 900 & 0 & 830 & 13.3 & 110 & 0.022 \\
\hline June-07 & 1,150 & 0 & 120 & 14.4 & 17 & 0.003 \\
\hline July-07 & 1,110 & 110 & 560 & 13.5 & 76 & 0.015 \\
\hline Aug.-07 & 960 & 60 & 650 & 13.3 & 87 & 0.018 \\
\hline Sept.-07 & 660 & 80 & 510 & 10.8 & 55 & 0.015 \\
\hline Oct. -07 & 360 & 0 & 620 & 8.6 & 53 & 0.018 \\
\hline Nov. -07 & 170 & 100 & 880 & 9.3 & 82 & 0.028 \\
\hline Dec. -07 & 50 & 90 & 940 & 10.0 & 94 & 0.029 \\
\hline Jan.-08 & 60 & 50 & 220 & 11.9 & 26 & 0.007 \\
\hline Feb. -08 & 140 & 100 & 200 & 7.7 & 15 & 0.007 \\
\hline Mar.-08 & 320 & 10 & 70 & 7.8 & 5 & 0.002 \\
\hline Apr.-08 & 530 & 0 & 50 & 8.0 & 4 & 0.001 \\
\hline May-08 & 750 & 50 & 700 & 8.5 & 59 & 0.018 \\
\hline June-08 & 1,140 & 10 & 520 & 13.8 & 72 & 0.014 \\
\hline July-08 & 1,120 & 110 & 270 & 14.2 & 38 & 0.007 \\
\hline Aug.-08 & 940 & 10 & 370 & 8.7 & 32 & 0.010 \\
\hline Sept.-08 & 650 & 20 & 870 & 12.8 & 111 & 0.027 \\
\hline Oct. -08 & 370 & 60 & 690 & 12.5 & 86 & 0.021 \\
\hline Nov.-08 & 160 & 80 & 640 & 11.6 & 75 & 0.021 \\
\hline Dec. -08 & 60 & 50 & 800 & 7.4 & 59 & 0.025 \\
\hline Jan.-09 & 100 & 50 & 680 & 11.2 & 76 & 0.020 \\
\hline Feb.-09 & 150 & 60 & 650 & 7.5 & 49 & 0.021 \\
\hline Mar.-09 & 360 & 0 & 860 & 7.7 & 66 & 0.023 \\
\hline Apr.-09 & 530 & 20 & 630 & 8.0 & 50 & 0.017 \\
\hline
\end{tabular}


Table 1. Reservoir data, evaporation, precipitation, and calculated recharge beneath Sand Hollow Reservoir, Washington County, Utah, 2002-16.-Continued

[Reservoir altitude and Reservoir storage: value is from the last day of each month; Reservoir surface area: value is an average of the daily values for each month; $2 o, 2$ standard deviation; ft/d, foot per day; —, no data available]

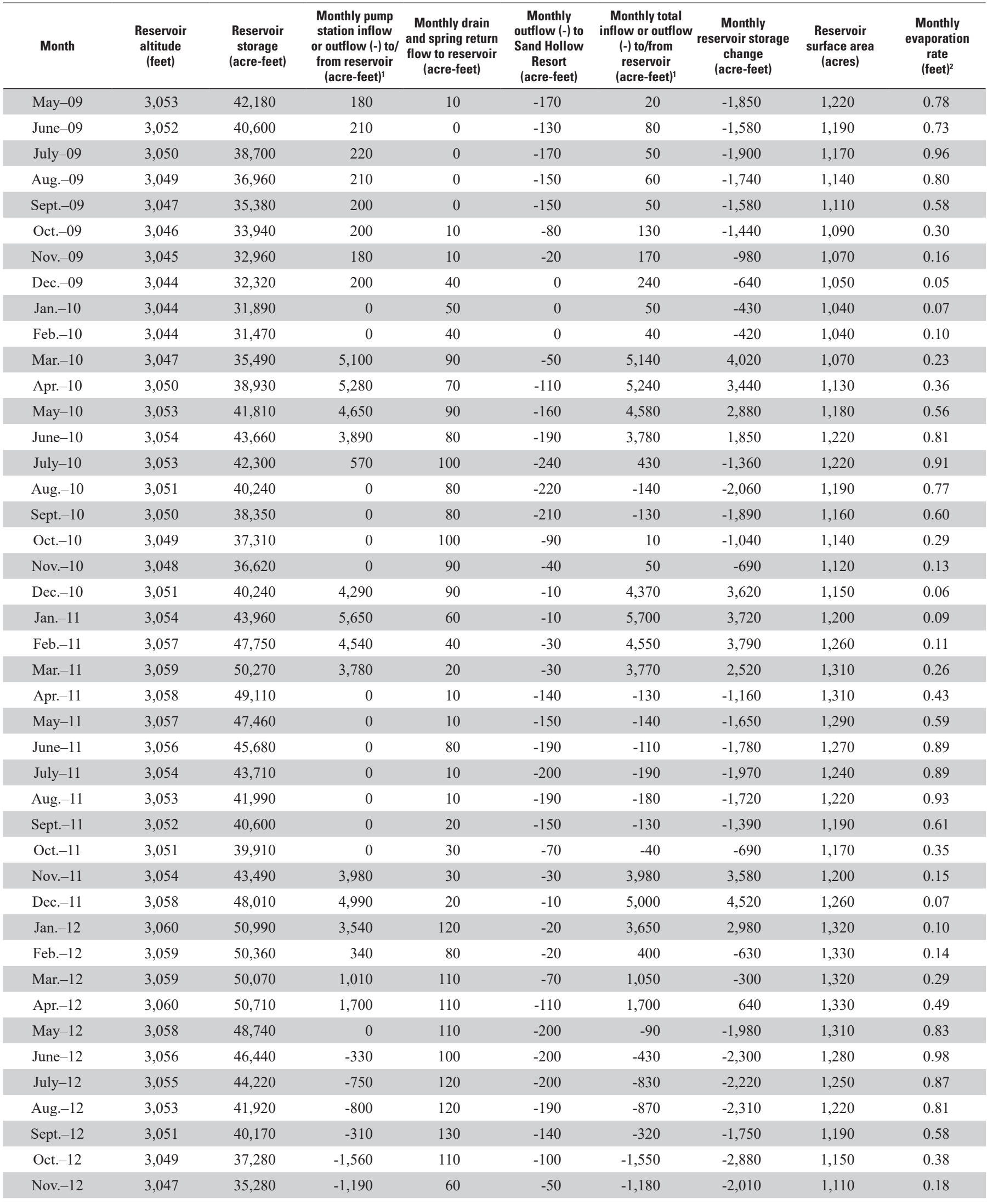


Table 1. Reservoir data, evaporation, precipitation, and calculated recharge beneath Sand Hollow Reservoir, Washington County, Utah, 2002-16. - Continued

[Reservoir altitude and Reservoir storage: value is from the last day of each month; Reservoir surface area: value is an average of the daily values for each month; 20,2 standard deviation; $\mathrm{ft} / \mathrm{d}$, foot per day; - , no data available]

\begin{tabular}{|c|c|c|c|c|c|c|}
\hline Month & $\begin{array}{c}\text { Monthly } \\
\text { evaporation } \\
\text { (acre-feet) }\end{array}$ & $\begin{array}{c}\text { Monthly } \\
\text { precipitation } \\
\text { (acre-feet) }\end{array}$ & $\begin{array}{l}\text { Monthly } \\
\text { groundwater } \\
\text { recharge } \\
\text { (acre-feet) }\end{array}$ & $\begin{array}{l}\text { Monthly } \\
\text { groundwater } \\
\text { recharge } \\
\text { uncertainty, 2 } \sigma \\
\text { (percent) }\end{array}$ & $\begin{array}{l}\text { Monthly } \\
\text { groundwater } \\
\text { recharge } \\
\text { uncertainty, 2 } \sigma \\
\text { (acre-feet) }\end{array}$ & $\begin{array}{l}\text { Groundwater } \\
\text { recharge rate } \\
\text { (ft/d) }\end{array}$ \\
\hline May-09 & 950 & 0 & 920 & 12.9 & 119 & 0.024 \\
\hline June-09 & 870 & 10 & 800 & 12.9 & 103 & 0.022 \\
\hline July-09 & 1,120 & 10 & 840 & 13.1 & 110 & 0.023 \\
\hline Aug.-09 & 910 & 0 & 890 & 12.8 & 114 & 0.025 \\
\hline Sept.-09 & 650 & 0 & 980 & 12.3 & 120 & 0.029 \\
\hline Oct.-09 & 320 & ${ }^{8} 0$ & 1,320 & 11.1 & 147 & 0.039 \\
\hline Nov.-09 & 170 & ${ }^{8} 0$ & 1,050 & 10.6 & 111 & 0.033 \\
\hline Dec.-09 & 60 & 100 & 920 & 9.6 & 88 & 0.028 \\
\hline Jan.-10 & 80 & 150 & 550 & 11.2 & 62 & 0.017 \\
\hline Feb.-10 & 110 & 220 & 570 & 11.5 & 65 & 0.018 \\
\hline Mar.-10 & 250 & 190 & 1,060 & 7.6 & 80 & 0.032 \\
\hline Apr.-10 & 400 & 40 & 1,440 & 7.6 & 109 & 0.041 \\
\hline May-10 & 660 & 10 & 1,050 & 8.0 & 84 & 0.029 \\
\hline June-10 & 990 & 0 & 940 & 8.6 & 81 & 0.025 \\
\hline July-10 & 1,100 & 0 & 690 & 12.7 & 88 & 0.018 \\
\hline Aug.-10 & 920 & 60 & 1,060 & 13.0 & 138 & 0.029 \\
\hline Sept. -10 & 690 & 0 & 1,070 & 12.7 & 136 & 0.030 \\
\hline Oct.-10 & 320 & 280 & 1,010 & 12.0 & 121 & 0.029 \\
\hline Nov.-10 & 150 & 80 & 670 & 11.6 & 78 & 0.019 \\
\hline Dec. -10 & 70 & 410 & 1,090 & 7.5 & 82 & 0.031 \\
\hline Jan.-11 & 100 & 10 & 1,890 & 7.1 & 135 & 0.051 \\
\hline Feb.-11 & 140 & 170 & 790 & 7.5 & 60 & 0.020 \\
\hline Mar.-11 & 340 & 110 & 1,020 & 7.7 & 79 & 0.025 \\
\hline Apr.-11 & 560 & 90 & 560 & 13.1 & 73 & 0.014 \\
\hline May-11 & 760 & 50 & 800 & 13.1 & 105 & 0.020 \\
\hline June-11 & 1,130 & 10 & 550 & 13.9 & 76 & 0.014 \\
\hline July-11 & 1,110 & 10 & 680 & 13.6 & 92 & 0.018 \\
\hline Aug.-11 & 1,130 & 20 & 430 & 13.9 & 60 & 0.011 \\
\hline Sept.-11 & 720 & 50 & 590 & 13.3 & 79 & 0.016 \\
\hline Oct.-11 & 420 & 120 & 350 & 13.4 & 47 & 0.010 \\
\hline Nov.-11 & 180 & 40 & 260 & 7.7 & 20 & 0.007 \\
\hline Dec. -11 & 90 & 50 & 440 & 7.5 & 33 & 0.011 \\
\hline Jan.-12 & 130 & 60 & 590 & 7.6 & 45 & 0.014 \\
\hline Feb.-12 & 180 & 150 & 1,010 & 10.1 & 102 & 0.024 \\
\hline Mar.-12 & 380 & 40 & 1,000 & 9.3 & 93 & 0.024 \\
\hline Apr.-12 & 650 & 60 & 450 & 9.4 & 42 & 0.011 \\
\hline May-12 & 1,090 & 0 & 800 & 13.6 & 109 & 0.020 \\
\hline June-12 & 1,260 & 40 & 650 & 12.8 & 83 & 0.016 \\
\hline July-12 & 1,090 & 200 & 500 & 11.7 & 58 & 0.013 \\
\hline Aug.-12 & 990 & 130 & 580 & 11.4 & 66 & 0.015 \\
\hline Sept.-12 & 690 & 110 & 850 & 11.9 & 101 & 0.023 \\
\hline Oct.-12 & 440 & 60 & 950 & 9.3 & 89 & 0.027 \\
\hline Nov.-12 & 200 & 0 & 630 & 8.8 & 56 & 0.018 \\
\hline
\end{tabular}


Table 1. Reservoir data, evaporation, precipitation, and calculated recharge beneath Sand Hollow Reservoir, Washington County, Utah, 2002-16.-Continued

[Reservoir altitude and Reservoir storage: value is from the last day of each month; Reservoir surface area: value is an average of the daily values for each month; 20,2 standard deviation; $\mathrm{ft} / \mathrm{d}$, foot per day; —, no data available]

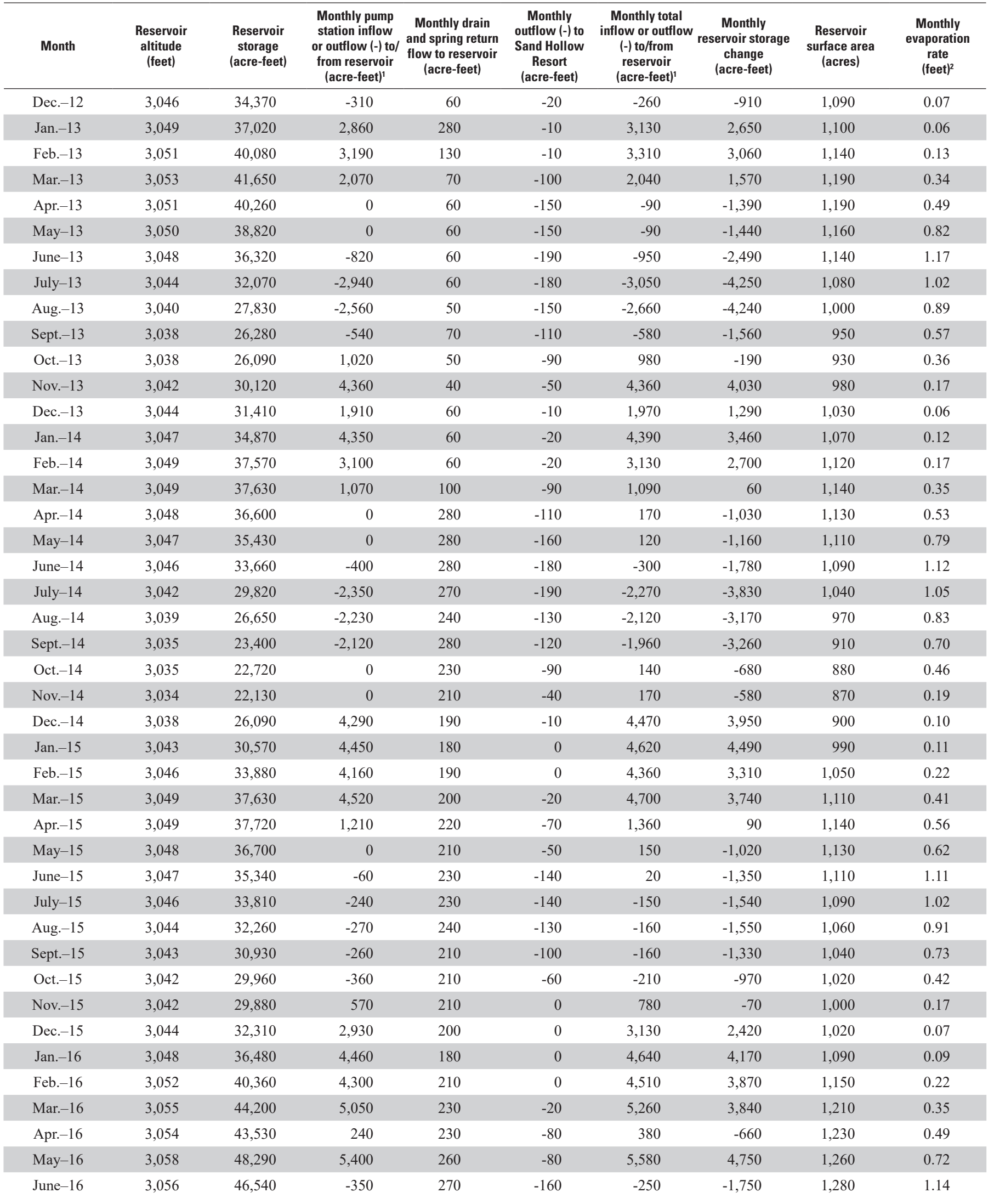


Table 1. Reservoir data, evaporation, precipitation, and calculated recharge beneath Sand Hollow Reservoir, Washington County, Utah, 2002-16. - Continued

[Reservoir altitude and Reservoir storage: value is from the last day of each month; Reservoir surface area: value is an average of the daily values for each month; $2 \alpha, 2$ standard deviation; ft/d, foot per day; -, no data available]

\begin{tabular}{|c|c|c|c|c|c|c|}
\hline Month & $\begin{array}{c}\text { Monthly } \\
\text { evaporation } \\
\text { (acre-feet) }\end{array}$ & $\begin{array}{c}\text { Monthly } \\
\text { precipitation } \\
\text { (acre-feet) }\end{array}$ & $\begin{array}{c}\text { Monthly } \\
\text { groundwater } \\
\text { recharge } \\
\text { (acre-feet) }\end{array}$ & $\begin{array}{l}\text { Monthly } \\
\text { groundwater } \\
\text { recharge } \\
\text { uncertainty, } 2 \sigma \\
\text { (percent) }\end{array}$ & $\begin{array}{c}\text { Monthly } \\
\text { groundwater } \\
\text { recharge } \\
\text { uncertainty, 2 } \sigma \\
\text { (acre-feet) }\end{array}$ & $\begin{array}{c}\text { Groundwater } \\
\text { recharge rate } \\
\text { (ft/d) }\end{array}$ \\
\hline Dec. -12 & 80 & 100 & 670 & 9.4 & 64 & 0.020 \\
\hline Jan.-13 & 70 & 90 & 500 & 7.6 & 38 & 0.015 \\
\hline Feb.-13 & 150 & 30 & 130 & 7.7 & 10 & 0.004 \\
\hline Mar.-13 & 400 & 40 & 110 & 8.5 & 9 & 0.003 \\
\hline Apr.-13 & 580 & 30 & 750 & 12.9 & 97 & 0.021 \\
\hline Мay-13 & 950 & 10 & 420 & 14.0 & 58 & 0.012 \\
\hline June-13 & 1,330 & 0 & 210 & 12.0 & 25 & 0.006 \\
\hline July-13 & 1,100 & 150 & 250 & 9.6 & 24 & 0.007 \\
\hline Aug.-13 & 890 & 80 & 760 & 9.5 & 73 & 0.025 \\
\hline Sept. -13 & 540 & 170 & 600 & 11.0 & 66 & 0.020 \\
\hline Oct.-13 & 340 & 20 & 860 & 8.9 & 77 & 0.030 \\
\hline Nov.-13 & 170 & 110 & 260 & 7.7 & 20 & 0.009 \\
\hline Dec. -13 & 60 & 50 & 660 & 7.3 & 48 & 0.021 \\
\hline Jan.-14 & 130 & 10 & 810 & 7.4 & 60 & 0.025 \\
\hline Feb.-14 & 190 & 120 & 350 & 7.8 & 28 & 0.010 \\
\hline Mar.-14 & 390 & 50 & 690 & 9.1 & 63 & 0.019 \\
\hline Apr.-14 & 600 & 50 & 650 & 13.6 & 89 & 0.019 \\
\hline May-14 & 880 & 110 & 520 & 14.1 & 73 & 0.015 \\
\hline June-14 & 1,210 & 0 & 260 & 13.0 & 34 & 0.008 \\
\hline July-14 & 1,100 & 10 & 480 & 9.9 & 47 & 0.015 \\
\hline Aug.-14 & 810 & 180 & 420 & 9.5 & 40 & 0.014 \\
\hline Sept.-14 & 640 & 140 & 800 & 9.3 & 75 & 0.029 \\
\hline Oct.-14 & 410 & 0 & 410 & 13.8 & 56 & 0.015 \\
\hline Nov.-14 & 160 & 20 & 600 & 12.1 & 73 & 0.022 \\
\hline Dec. -14 & 90 & 60 & 490 & 7.5 & 37 & 0.017 \\
\hline Jan.-15 & 110 & 70 & 100 & 7.7 & 8 & 0.003 \\
\hline Feb.-15 & 230 & 30 & 840 & 7.6 & 64 & 0.026 \\
\hline Mar.-15 & 460 & 140 & 640 & 8.0 & 51 & 0.019 \\
\hline Apr.-15 & 640 & 40 & 660 & 10.2 & 68 & 0.019 \\
\hline May-15 & 700 & 60 & 530 & 13.9 & 74 & 0.015 \\
\hline June-15 & 1,240 & 10 & 150 & 14.5 & 22 & 0.004 \\
\hline July-15 & 1,100 & 60 & 340 & 13.4 & 45 & 0.010 \\
\hline Aug.-15 & 960 & 70 & 500 & 12.9 & 65 & 0.015 \\
\hline Sept. -15 & 760 & 60 & 470 & 12.6 & 60 & 0.015 \\
\hline Oct.-15 & 430 & 80 & 410 & 11.4 & 47 & 0.013 \\
\hline Nov. -15 & 170 & 50 & 730 & 8.7 & 64 & 0.024 \\
\hline Dec. -15 & 70 & 40 & 670 & 7.5 & 50 & 0.021 \\
\hline Jan.-16 & 90 & 130 & 500 & 7.6 & 38 & 0.015 \\
\hline Feb.-16 & 250 & 60 & 440 & 7.8 & 34 & 0.012 \\
\hline Mar.-16 & 420 & 90 & 1,100 & 7.8 & 85 & 0.029 \\
\hline Apr.-16 & 600 & 200 & 650 & 12.8 & 84 & 0.017 \\
\hline May-16 & 900 & 90 & 10 & 8.4 & 1 & 0.000 \\
\hline June-16 & 1,460 & 10 & 50 & 13.6 & 7 & 0.001 \\
\hline
\end{tabular}


Table 1. Reservoir data, evaporation, precipitation, and calculated recharge beneath Sand Hollow Reservoir, Washington County, Utah, 2002-16.-Continued

[Reservoir altitude and Reservoir storage: value is from the last day of each month; Reservoir surface area: value is an average of the daily values for each month; 20,2 standard deviation; $\mathrm{ft} / \mathrm{d}$, foot per day; - , no data available]

\begin{tabular}{|c|c|c|c|c|c|c|c|c|c|}
\hline Month & $\begin{array}{l}\text { Reservoir } \\
\text { altitude } \\
\text { (feet) }\end{array}$ & $\begin{array}{l}\text { Reservoir } \\
\text { storage } \\
\text { (acre-feet) }\end{array}$ & $\begin{array}{l}\text { Monthly pump } \\
\text { station inflow } \\
\text { or outflow (-) to/ } \\
\text { from reservoir } \\
\text { (acre-feet) }^{1}\end{array}$ & $\begin{array}{l}\text { Monthly drain } \\
\text { and spring return } \\
\text { flow to reservoir } \\
\text { (acre-feet) }\end{array}$ & $\begin{array}{c}\text { Monthly } \\
\text { outflow (-) to } \\
\text { Sand Hollow } \\
\text { Resort } \\
\text { (acre-feet) }\end{array}$ & $\begin{array}{l}\text { Monthly total } \\
\text { inflow or outflow } \\
\text { (-) to/from } \\
\text { reservoir } \\
\text { (acre-feet) }^{1}\end{array}$ & $\begin{array}{c}\text { Monthly } \\
\text { reservoir storage } \\
\text { change } \\
\text { (acre-feet) }\end{array}$ & $\begin{array}{l}\text { Reservoir } \\
\text { surface area } \\
\text { (acres) }\end{array}$ & $\begin{array}{c}\text { Monthly } \\
\text { evaporation } \\
\text { rate } \\
{\text { (feet })^{2}}^{2}\end{array}$ \\
\hline July-16 & 3,054 & 43,780 & -930 & 280 & -100 & -750 & $-2,760$ & 1,250 & 1.18 \\
\hline Aug.-16 & 3,053 & 42,090 & -160 & 250 & -160 & -60 & $-1,700$ & 1,220 & 0.93 \\
\hline Sept.-16 & 3,052 & 40,710 & -100 & 260 & -100 & 70 & $-1,380$ & 1,190 & 0.64 \\
\hline Oct.-16 & 3,051 & 39,560 & 0 & 260 & -60 & 200 & $-1,150$ & 1,170 & 0.44 \\
\hline Nov.-16 & 3,051 & 39,560 & 1,520 & 240 & 0 & 1,760 & 0 & 1,160 & 0.22 \\
\hline Dec. -16 & 3,052 & 40,730 & 1,690 & 240 & 0 & 1,930 & 1,170 & 1,170 & 0.08 \\
\hline Total & - & - & - & - & - & 257,460 & - & - & - \\
\hline
\end{tabular}

Table 1. Reservoir data, evaporation, precipitation, and calculated recharge beneath Sand Hollow Reservoir, Washington County, Utah, 2002-16.-Continued

[Reservoir altitude and Reservoir storage: value is from the last day of each month; Reservoir surface area: value is an average of the daily values for each month; 20,2 standard deviation; $\mathrm{ft} / \mathrm{d}$, foot per day; -, no data available]

\begin{tabular}{|c|c|c|c|c|c|c|}
\hline Month & $\begin{array}{c}\text { Monthly } \\
\text { evaporation } \\
\text { (acre-feet) }\end{array}$ & $\begin{array}{c}\text { Monthly } \\
\text { precipitation } \\
\text { (acre-feet) }\end{array}$ & $\begin{array}{l}\text { Monthly } \\
\text { groundwater } \\
\text { recharge } \\
\text { (acre-feet) }\end{array}$ & $\begin{array}{l}\text { Monthly } \\
\text { groundwater } \\
\text { recharge } \\
\text { uncertainty, } 2 \sigma \\
\text { (percent) }\end{array}$ & $\begin{array}{l}\text { Monthly } \\
\text { groundwater } \\
\text { recharge } \\
\text { uncertainty, } 2 \sigma \\
\text { (acre-feet) }\end{array}$ & $\begin{array}{c}\text { Groundwater } \\
\text { recharge rate } \\
\text { (ft/d) }\end{array}$ \\
\hline July-16 & 1,480 & 60 & 590 & 11.9 & 70 & 0.015 \\
\hline Aug.-16 & 1,130 & 160 & 670 & 13.3 & 89 & 0.018 \\
\hline Sept. -16 & 760 & 140 & 830 & 13.0 & 107 & 0.022 \\
\hline Oct.-16 & 510 & 40 & 880 & 13.0 & 114 & 0.024 \\
\hline Nov.-16 & 250 & 60 & 1,570 & 7.2 & 114 & 0.044 \\
\hline Dec. -16 & 90 & 230 & 900 & 7.6 & 69 & 0.025 \\
\hline Total & 84,950 & - & 140,740 & - & - & - \\
\hline
\end{tabular}

${ }^{1}$ Negative (-) values indicate flows out of Sand Hollow Reservoir to Quail Creek Water Treatment Plant or to Quail Creek Reservoir.

${ }^{2}$ Monthly evaporation rate from February 2007 through December 2009 was calculated with a correction factor to account for higher solar radiation measurements with new instrument. ${ }^{3}$ Because of problems with monitoring equipment, inflows from September 2004 through February 2005 are estimated based on previous inflow history and changes in reservoir altitude. ${ }^{4}$ Revised value based on refined reservoir altitude estimate for October 2005.

${ }^{5}$ Monthly pump station outflow was increased from amount reported in Heilweil, Ortiz, and Susong (2009) based on reservoir altitude relations.

${ }^{6}$ Monthly total outflow was increased from amount reported by Washington County Water Conservancy District based on reservoir altitude relations.

${ }^{7}$ Monthly pump station outflow was increased from amount reported in Heilweil, Ortiz, and Susong (2009) based on reservoir altitude relations.

${ }^{8}$ Sand Hollow rain gage not functioning; values of 0 based on lack of precipitation from St. George precipitation station no. 427516.

${ }^{9}$ Monthly total inflow or outflow (-) to/from reservoir (acre-feet). 


\section{Meteorological Data}

Meteorological data have been collected at the WCWCD weather station (fig. 2) in Sand Hollow since January 1998. Beginning in 2010, a Soil Climate Analysis Network (SCAN) Sand Hollow weather station (http://www.wcc.nrcs.usda.gov/ nwcc/site?sitenum=2159) operated by the Natural Resources Conservation Service (NRCS), replaced the collection of data by the WCWCD weather station. The NRCS Sand Hollow SCAN station is located south of the reservoir within Sand Hollow basin near the WCWCD station (fig. 2). Data from both weather stations have been used for evaluating evaporation and precipitation, which are required for calculating monthly recharge from Sand Hollow Reservoir. Parameters measured include air temperature, wind speed, wind direction, precipitation, relative humidity, and incoming solar radiation. Instrumentation includes a temperature and relative humidity probe, a wind direction and speed monitor, a tipping bucket rain gage, and a solar radiometer. Sensors collect data every minute, and average hourly and daily values are computed and stored on a data logger, with the exception of precipitation, which is summed rather than averaged. The solar radiation and temperature data were used for calculating evaporation by using the version of the Jenson-Haise method found in McGuinness and Bordne (1971). The other data were collected to permit calculations of evaporation using other methods.

From January 13, 1998, to December 31, 2016, daily average air temperature ranged from -10 to 36 degrees Celsius $\left({ }^{\circ} \mathrm{C}\right)$. The coldest temperatures during the year were typically in December and January, when minimum air temperatures occasionally were below $-10^{\circ} \mathrm{C}$. The warmest temperatures were typically in July, when maximum air temperatures occasionally approached $45^{\circ} \mathrm{C}$. Daily average solar radiation ranged from 14 to 990 calories per square centimeter per day. The minimum daily averages are typically in December and January, and the maximum daily averages are typically in June and July.

Monthly precipitation was recorded at the Sand Hollow weather station continuously from January 1998 through December 2016, except for two periods when malfunctioning instrumentation resulted in data loss: December 26, 2008, to January 3, 2009, and September 28 to November 16, 2009. Precipitation amounts during these two periods were estimated on the basis of data from the nearby St. George Southgate Golf Course weather station (no. 427516; http://www.wrcc.dri. edu/cgi-bin/cliMAIN.pl?ut7516). From January 1998 through December 2016, monthly precipitation ranged from 0 to about 4.3 in. (fig. 7) and averaged about 0.6 in. Average annual precipitation during the 19 -year period was $7.7 \mathrm{in}$.

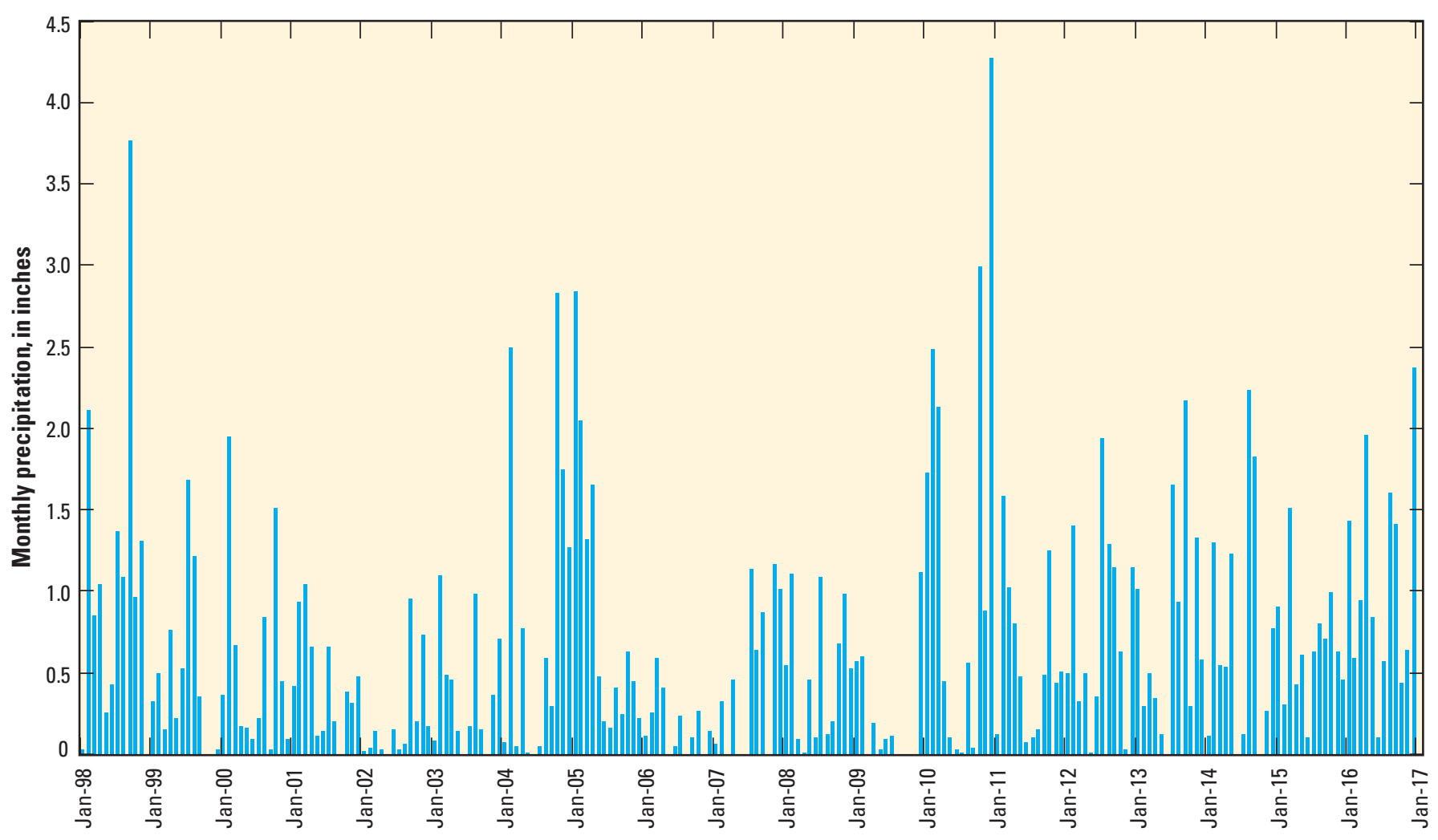

Figure 7. Monthly precipitation at Sand Hollow Reservoir, Washington County, Utah, 1998-2016. 


\section{Estimates of Managed Aquifer Recharge From Sand Hollow Reservoir}

Substantial amounts of surface water from Sand Hollow Reservoir infiltrate the underlying sediments to recharge the Navajo aquifer. This recharge either is captured by production wells for municipal supply, or it moves northward through the aquifer towards the Virgin River. Through 2016, withdrawals from production wells operated by the WCWCD at Sand Hollow have been permitted for the capture of natural recharge in Sand Hollow. These withdrawals are governed by different water rights than those associated with MAR from Sand Hollow Reservoir; withdrawal rights for this artificial recharge have not yet been exercised.

Recharge from Sand Hollow Reservoir is calculated as the residual with the following water-budget equation (modified from Heilweil and others, 2005):

$$
R=I_{s w}+I_{d r}-O_{s w}+P \pm \Delta S-E
$$

where

$$
\begin{aligned}
R & \text { is recharge, } \\
I_{s w} & \text { is surface-water inflow, } \\
I_{d r} & \text { is drain return flow, } \\
O_{s w} & \text { is surface-water outflow, } \\
P & \text { is the amount of precipitation falling directly } \\
& \quad \text { on the reservoir, } \\
\Delta S & \text { is change in surface-water storage, and } \\
E & \text { is evaporation. }
\end{aligned}
$$

All the variables in equation 1 are in units of acre-feet.

The following equation was developed to evaluate the uncertainty for each monthly recharge estimate:

$$
C U=\Sigma\left[\left(\left|C_{i}\right| / \Sigma\left|C_{i}\right|\right) * U_{i}\right]
$$

where

$$
\begin{array}{r}
C U \quad \begin{array}{r}
\text { is the composite uncertainty fraction (2 } \\
\text { standard deviation, } 2 \sigma),
\end{array} \\
\left|C_{i}\right| \quad \begin{array}{r}
\text { is the absolute value of each component of the } \\
\text { water budget (acre-feet), }
\end{array} \\
\Sigma\left|C_{i}\right| \quad \begin{array}{r}
\text { is the sum of absolute values of all the water- } \\
\text { budget components (acre-feet), and }
\end{array} \\
U_{i} \quad \begin{array}{r}
\text { is the uncertainty fraction (2 } \sigma) \text { for each } \\
\text { individual water-budget component. }
\end{array}
\end{array}
$$

The smallest estimated uncertainty fraction is 0.05 (5 percent) for $I_{s w}, I_{d r}$, and $O_{s w}$ because these flows are recorded using calibrated inline flow meters. The estimated uncertainty fraction for $P$ is higher, at 0.10 (10 percent), because it is an indirect measurement made on the basis of nearby meteorological station data. Similarly, the estimated uncertainty fraction is also 0.10 (10 percent) for $\Delta S$ because changes in surface-water storage are based only on approximate reservoir water-level altitude/volume relations rather than direct measurements. The largest estimated uncertainty fraction is 0.20 (20 percent) for $E$, which is based on differences between alternative methods for estimating evaporation at Sand Hollow and other areas (Heilweil and others, 2007; Rosenberry and others, 2007).

The first two reports documenting monthly groundwater recharge beneath Sand Hollow Reservoir through August 2006 (Heilweil and others, 2005; Heilweil and Susong, 2007) did not include precipitation falling directly on the reservoir. Beginning with the third report (Heilweil, Ortiz, and Susong, 2009), and continuing in this report, an additional term for precipitation falling directly on the reservoir $(P)$ was included in equation 1 . The monthly amount of precipitation falling on the reservoir is calculated by multiplying the total monthly precipitation recorded by the Sand Hollow weather station by the average reservoir surface area for that month, based on reservoir water-level altitude to area relations for the reservoir (RBG Engineering, written commun., 2002; Washington County Water Conservancy District, written commun., 2006). The precipitation term in equation 1 , however, does not account for precipitation runoff to the reservoir. Because of high evaporation rates and permeable surficial soils, precipitation events seldom produce runoff that reaches the lower part of Sand Hollow (L. Jessop, Washington County Water Conservancy District, oral commun., 2001), where the reservoir is situated.

Monthly water-budget values for Sand Hollow Reservoir are shown in table 1 . Values are generally monthly averages or totals, except for reservoir altitude and storage, which are shown for the last day of each month. Values for "Monthly evaporation rate," "Monthly evaporation," and "Monthly groundwater recharge" from March 2002 through January 2005, and from January 2008 through December 2016, are monthly averages. During February 2005 through December 2007, however, the values are the sum of daily measurements. Summing of daily evaporation estimates was discontinued after 2007 because a comparison of daily and average monthly calculations during 2008 and 2009 showed little difference, and the equation used for calculating evapotranspiration is more appropriate for calculating average evaporation over longer periods (McGuinness and Bordne, 1971).

\section{Changes in Reservoir Storage}

Changes in reservoir storage were calculated from daily reservoir water-level altitudes reported by the WCWCD using altitude to volume relations (RBG Engineering, written commun., 2002). Since inception of the reservoir in 2002, surface-water storage has increased to a maximum of about 51,000 acre-ft in May 2006. From the latter half of 2006 through 2007, surface-water storage decreased to about 32,000 acre-ft, and during 2008 through 2010, surface-water storage varied between about 31,000 and 44,000 acre-ft. Following the abnormally wet winter and spring of 2010-11, surface-water storage was kept at a high level during 2011 through mid-2012, varying between about 40,000 and 
51,000 acre-ft, but then declined to as little as about 22,000 acre-ft in November 2014 as a result of less than normal precipitation in the region and decreased flows in the Virgin River (table 1). Surface-water storage increased again in 2016, reaching about 48,000 acre-ft in May, the highest level since spring 2012.

\section{Reservoir Evaporation}

The McGuinness and Bordne (1971) version of the Jensen-Haise method was selected for calculating evaporation from Sand Hollow Reservoir during this study. A detailed comparison to results using other methods for estimating evaporation is given in Heilweil and others (2005). The McGuinness and Bordne (1971) version of the Jensen-Haise method is based on the following relation:

$$
P E T=\left\{\left[\left(\left(0.01 T_{a}\right)-0.37\right)\left(Q_{s}\right)\right] 0.000673\right\} 2.54
$$

where

$$
\begin{array}{cl}
\text { PET } & \begin{array}{c}
\text { is potential evaporation, in centimeters per } \\
\text { day, }
\end{array} \\
T_{a} & \text { is air temperature, in degrees Fahrenheit, and } \\
Q_{s} & \begin{array}{c}
\text { is solar radiation, in calories per square } \\
\text { centimeter per day. }
\end{array}
\end{array}
$$

The units for PET can be converted to feet per day by multiplying by 0.0328 .
By using air temperature and solar radiation from the nearby SCAN weather station (fig. 2), monthly evaporation rates were calculated with equation 3 . These estimated evaporation rates ranged from 0.04 to 1.18 feet per month from March 2002 through December 2016 (table 1). Multiplying the estimated evaporation rates by average reservoir surface area yielded monthly evaporation losses that ranged from about 20 to 1,500 acre-ft between March 2002 and December 2016.

\section{Estimates of Total Volume of Managed Aquifer Recharge From Sand Hollow Reservoir}

Monthly estimates of precipitation $(P)$, evaporation $(E)$, inflows $\left(I_{s w}\right.$ and $\left.I_{d r}\right)$, outflows $\left(O_{s w}\right)$, and changes in surfacewater storage $(\Delta S)$ were used in equation 1 to calculate recharge to the Navajo aquifer beneath Sand Hollow Reservoir. Monthly recharge from March 2002 through December 2016 ranged from about 12 to 3,500 acre-ft (fig. 8, table 1), with 2 standard deviation $(\sigma)$ composite uncertainties ranging from about 6 to 15 percent of the estimate. Monthly recharge from January 2015 through December 2016 ranged from about 12 to 1,600 acre-ft, with similar composite uncertainties. Higher composite uncertainties in the summer reflect the larger, weighted importance of evaporation losses, which have the highest uncertainty.

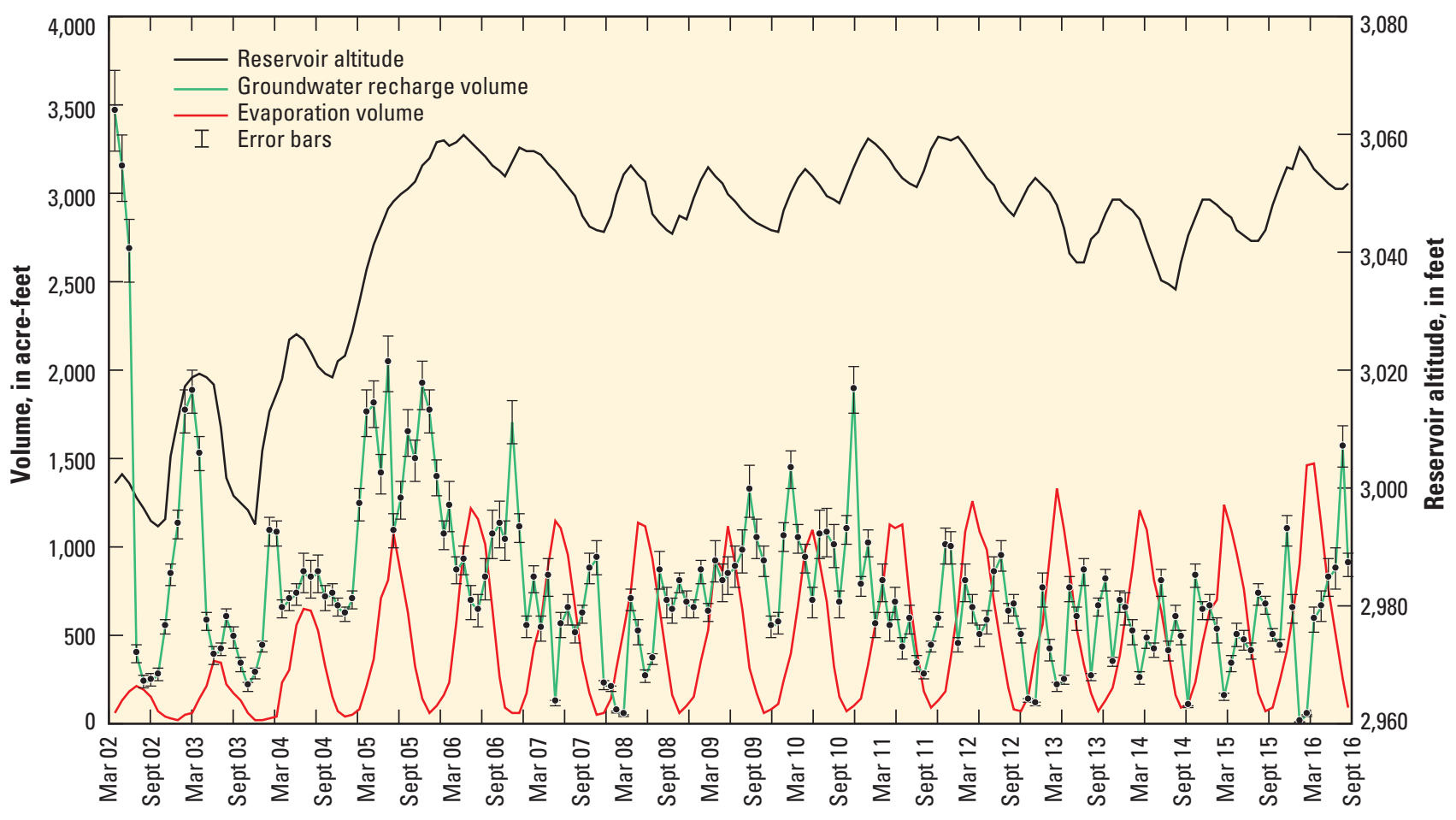

Figure 8. Monthly estimated evaporation and groundwater recharge in relation to reservoir altitude at Sand Hollow Reservoir, Washington County, Utah, 2002-16. 


\section{Assessment of Managed Aquifer Recharge at Sand Hollow Reservoir, Washington County, Utah}

Estimated average monthly recharge rates beneath Sand Hollow Reservoir ranged from about 0.000 to 0.430 foot per day (ft/d) between March 2002 and December 2016 (fig. 9). Although the graph shows large monthly fluctuations, recharge has generally stabilized at an average of about $0.02 \mathrm{ft} / \mathrm{d}$ during 2011 through 2016.

Net annual inflow, evaporation, and groundwater recharge from Sand Hollow Reservoir from 2002 through 2016 are shown on figure 10 . Total net inflow during this period was about 256,000 acre-ft, with annual inflow ranging from about 800 acre-ft in 2007 to 56,000 acre-ft in 2005. The general increase in reservoir water-level altitude and area from 2002 to 2007 resulted in a steady increase in the volume of annual evaporation from about 1,100 to 6,600 acre-ft over the same period, and then remained relatively constant, ranging between 5,700 acre-ft in 2010 and 7,900 acre-ft in 2016. Total estimated evaporative losses from 2002 through 2016 were about 85,000 acre-ft. Total estimated evaporation losses from 2015 through 2016 were about 14,800 acre-ft. Annual recharge ranged from a low of about 5,400 acre-ft in 2008 to a high of about 18,000 acre-ft in 2005. Total estimated recharge from 2002 through 2016 was about 141,000 acre-ft, with a two standard deviation uncertainty of 13,300 acre-ft. Of that total, about 14,200 acre-ft of recharge occurred in 2015 and 2016.

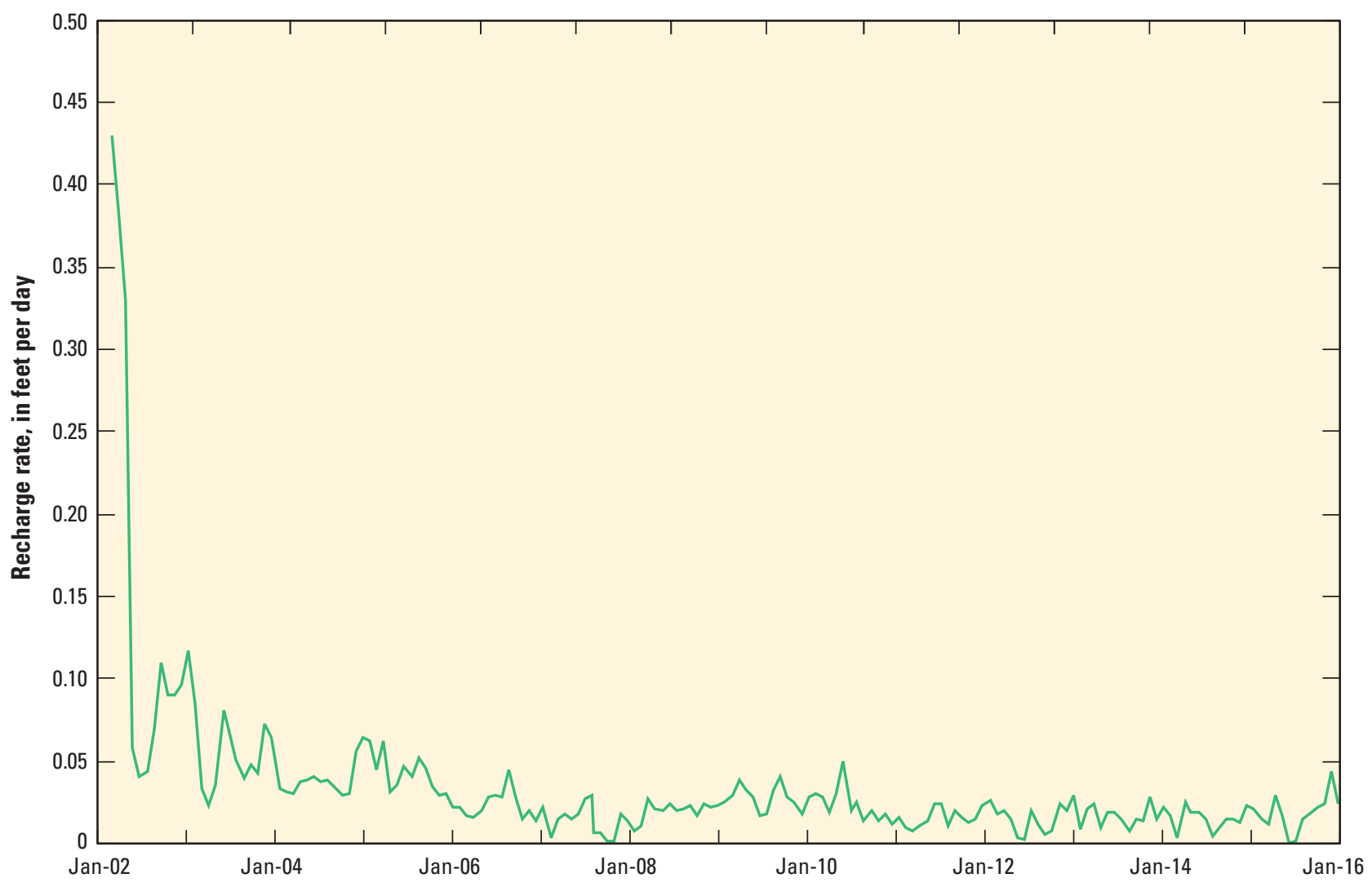

Figure 9. Monthly calculated groundwater recharge rates beneath Sand Hollow Reservoir, Washington County, Utah, 2002-16. 


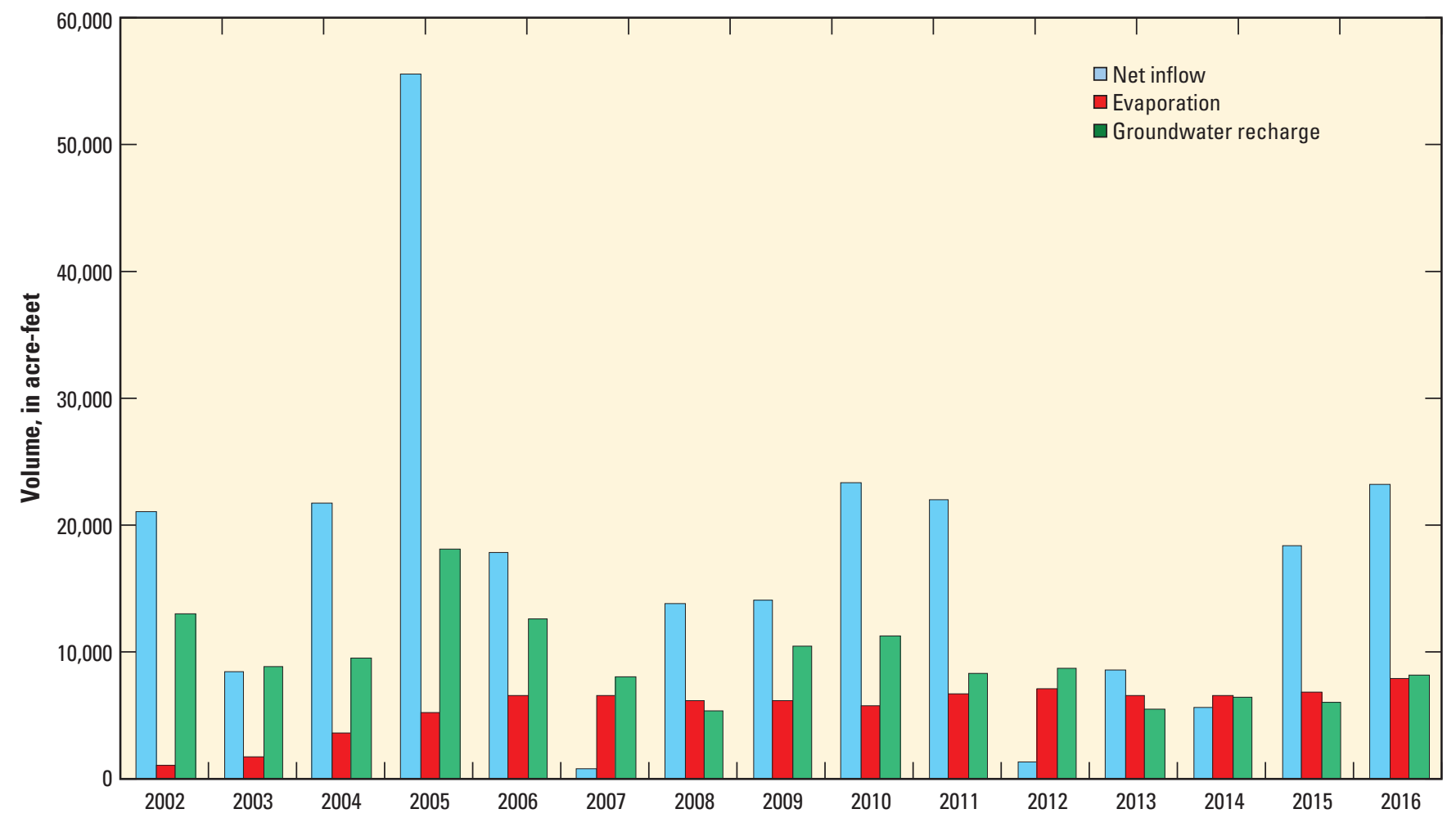

Figure 10. Estimated annual net inflow, evaporation, and groundwater recharge at Sand Hollow Reservoir, Washington County, Utah, 2002-16. 


\section{Groundwater and Surface-Water Quality in Sand Hollow}

As MAR from Sand Hollow Reservoir moves into the underlying Navajo aquifer, it has an initial water-quality signature similar to the reservoir water, but this evolves as water moves through the subsurface. Along its flow path, the MAR initially moves from the reservoir through the organicrich silt layer that has accumulated beneath the reservoir, and then through the pre-reservoir vadose zone (now saturated) where vadose-zone solutes had naturally accumulated and air was trapped prior to and during filling of the reservoir. This results in water quality that is different from native groundwater. As part of the monitoring of MAR from Sand Hollow Reservoir, water-quality samples from the reservoir and surrounding monitoring wells were collected and analyzed for field water-quality parameters and laboratory chemical, isotopic, and dissolved-gas concentrations.

Field water-quality parameters include water temperature, specific conductance, $\mathrm{pH}$, dissolved oxygen (DO), and total dissolved-gas (TDG) pressure. Total dissolved-gas pressure is the combination of partial pressures of all dissolved gases in the water. Field parameters were measured with a multiparameter sonde placed within the screened interval at the bottom of each 2-inch-diameter monitoring well, and in the reservoir at depths of approximately $2 \mathrm{ft}$. The multiparameter sonde was too large to enter the 1-inch-diameter monitoring wells (North Dam 3A, WD 4, WD 5, and WD 12). Consequently, field measurements from these wells were made onsite using a flow-through chamber connected to the discharge line from either a Waterra inertia pump or peristaltic pump; no TDG pressure measurements were made at these sites. Additional details regarding field parameter methods are given in Heilweil and others (2005) and Heilweil and Susong (2007).

Laboratory analyses of water from Sand Hollow Reservoir and groundwater from the Navajo aquifer included dissolved major ions and trace elements, dissolved organic carbon (DOC), tritium $\left({ }^{3} \mathrm{H}\right)$, and industrial dissolved gases. The major inorganic ions included calcium, magnesium, sodium, potassium, bicarbonate, sulfate, chloride, fluoride, and nitrate. Trace elements included bromide, iron, manganese, arsenic, nitrite, ammonia, and orthophosphate. Dissolved gases included chlorofluorocarbons (CFC-11, CFC-12, CFC-113) and sulfur hexafluoride ( $\mathrm{SF}_{6}$ ).

Water samples were collected from the 2-inch-diameter monitoring wells by using either a Grundfos or Bennett submersible pump; 1-inch-diameter wells were sampled with an inertia pump, and production wells were sampled using installed turbine pumps. Prior to sample collection from monitoring wells, the water was purged from each well until field parameters stabilized and a minimum of three casing volumes were removed. After purging each well, water was pumped into sample bottles and filtered as required. Since 2009 , a set of replicate samples has been collected annually at one randomly selected site and separately analyzed for all constituents for quality assurance. Samples for major ions and trace elements were filtered through 0.45 -micron disposable filters and collected in pre-cleaned polyethylene bottles according to procedures described by Wilde and Radtke (1998); samples for analysis of cations (calcium, magnesium, sodium, and potassium) and trace elements were preserved with 7.7-normal nitric acid. Tritium samples were collected in 500-milliliter $(\mathrm{ml})$ polyethylene bottles with polyseal caps and no head space. Samples for $\mathrm{CFC}$ and $\mathrm{SF}_{6}$ analysis were collected in 250-ml and 1-liter glass bottles, respectively, according to procedures described on the USGS Reston Groundwater Dating Laboratory website at http://water.usgs. gov/lab/.

Inorganic and organic chemical analyses (major ions, trace elements, DOC) were analyzed at the USGS National Water Quality Laboratory in Denver, Colorado. Samples collected during 2008-12 and 2015-16 for CFCs and $\mathrm{SF}_{6}$ were analyzed by the USGS Chlorofluorocarbon Laboratory in Reston, Virginia. Tritium and $\mathrm{SF}_{6}(2013-14)$ were analyzed at the University of Utah Dissolved Gas Service Center in Salt Lake City.

\section{Water-Quality Results}

Detailed water-quality data and interpretations for Sand Hollow, including trends through 2012, have been published previously (Heilweil and others, 2005; Heilweil and Susong, 2007; Heilweil, Ortiz, and Susong, 2009; Heilweil and Marston, 2011; Marston and Heilweil, 2013; Marston and Heilweil, 2016). Tables 2 and 3 provide these previous data, along with additional data collected during 2014 and 2016. The following discussion describes recent changes (2015-16) or the continuation of longer trends (2002-16) in water quality.

The most significant changes in water quality have occurred at wells WD 4 and WD 12, located 2,600 and $1,000 \mathrm{ft}$ from the reservoir, respectively. Although the low chloride/bromide ratios indicate that reservoir water has not yet arrived at these sites, increases in other constituent concentrations indicate a flush of naturally accumulating vadose-zone salts ahead of reservoir recharge. At well WD 4, specific conductance and dissolved-solids concentrations increased from 2011 through 2016 to values similar to those in the reservoir water. Although concentrations of environmental tracers (tritium, $\mathrm{CFCs}$, and $\mathrm{SF}_{6}$ ) have also increased, they are still lower than those in the reservoir water. At well WD 12, specific conductance and dissolved-solids concentrations increased from 2011 through 2016 to higher values than those in the reservoir, indicating mobilization of naturally accumulating vadose-zone salts; however, chloride/bromide ratios and environmental tracer concentrations (tritium, CFCs, and $\mathrm{SF}_{6}$ ) were still lower than those in the reservoir water.

At well WD 6, located 1,000 ft from the reservoir, the increase in the chloride/bromide ratio from 2002 through 2016 toward ratios in the reservoir water indicates the arrival of recharge from the reservoir. Specific conductance, dissolved-solids concentrations, and concentrations of some environmental tracers (tritium, CFC-12, CFC-113) are also 
about the same as those in the reservoir water and have remained relatively stable from 2012 through 2016. Other industrial gases (CFC-11, $\left.\mathrm{SF}_{6}\right)$ and DOC concentrations are substantially lower than reservoir concentrations, but this may indicate other processes or environmental conditions that are unique to this site.

Although water from well WD 8 has shown a slight increase in the chloride/bromide ratio from 2002 through 2016, it is still much lower than the ratio in the reservoir water. Located only $500 \mathrm{ft}$ from the reservoir, this well is downgradient of an area of high natural recharge on Sand Mountain and may lie along a groundwater boundary between the two recharge mounds. The relatively high concentrations of modern environmental tracers may be caused by natural recharge. Further, an increased concentration in DO to $26 \mathrm{mg} / \mathrm{L}$ indicates a rapid rise in water level and entrapment of air bubbles.

At wells WD 9 and WD 11, 55 and $160 \mathrm{ft}$ away from the reservoir, respectively, the various field parameters, along with the results of chemical analyses and environmental tracers (with the exception of CFC-11 and CFC-113, which were possibly reduced by microbial degradation) indicate that reservoir recharge arrived several years prior to 2014. Recent declines in DO in these wells may be due to a combination of dissolution of trapped air bubbles and chemical reduction as recharge infiltrates through carbon-rich sediment at the bottom of the reservoir. At well WD 22, 1,650 ft from the reservoir, increases in specific conductance and chloride/bromide ratios indicate arrival of some component of reservoir recharge prior to well drilling and completion in the summer of 2015. Results from environmental tracer analyses also indicate increasing concentrations of tritium and CFCs. The location of well WD 22 was based on predicted travel times from a numerical model of the Hurricane Bench that simulates recharge from Sand Hollow Reservoir (Marston and Heilweil, 2012).

At well WD 15, located 2,400 ft from the reservoir (fig. 2), specific conductance and dissolved-solids concentrations increased from 2010 through 2014, yet chloride/bromide ratios and tritium concentrations remained much lower than those in the reservoir water. This may indicate vadose-zone salt mobilization, likely from rising water levels rather than a salt flush prior to the arrival of reservoir water. Elevated TDG pressure and DO similarly indicate air entrapment associated with rising water levels. Well WD 16, located at the same site but screened at a deeper interval, does not show an increase in salinity and has even lower chloride/bromide ratios and tritium concentrations, supporting the interpretation of rising water levels rather than salt flushing prior to the arrival of reservoir recharge. Wells farther from the reservoir that were sampled in 2013 or 2014 (WD 5 located 2,800 ft away and wells WD 17 and WD 18 located 5,900 ft away) show that reservoir recharge has not yet reached these locations.

Arsenic concentrations have generally decreased at locations where reservoir recharge has already arrived or where there has been a water-table rise and flushing of vadosezone salts, typically a precursor to the arrival of recharge. This decline may be attributable to an increase in oxidizing conditions, which facilitate the adsorption of arsenic on iron oxides within the aquifer matrix, in contrast to reducing conditions, which would mobilize arsenic. Analyses of water from wells WD 4, WD 6, WD 8, and WD12 all show a trend of decreasing arsenic concentration with increasing DO.

Table 2. Field measurements, total dissolved-gas pressure, and concentrations of dissolved organic carbon, tritium, chlorofluorocarbons, and sulfur hexafluoride in groundwater and surface water from Sand Hollow, Washington County, Utah.

[Analyzing agency: Dissolved organic carbon at U.S. Geological Survey (USGS) National Water Quality Laboratory in Denver, Colorado; Tritium at University of Utah Dissolved Gas Laboratory in Salt Lake City, Utah; CFC-11, CFC-12, CFC-113, and $\mathrm{SF}_{6}$ at USGS Chlorofluorocarbon (CFC) Laboratory in Reston, Virginia. Abbreviations: ${ }^{\circ} \mathrm{C}$, degrees Celsius; $\mu \mathrm{S} / \mathrm{cm}$, microsiemens per centimeter at 25 degrees Celsius; $\mathrm{mg} / \mathrm{L}$, milligrams per liter; $\mathrm{mm} \mathrm{Hg}$, millimeters of mercury; TU, tritium units; pmol/kg, picomoles per kilogram; fmol/kg, femtomoles per kilogram; - , no data available; E, estimated; >, greater than; <, less than]

\begin{tabular}{|c|c|c|c|c|c|c|c|c|c|c|c|c|c|}
\hline Site name & $\begin{array}{c}\text { Date } \\
\text { sampled }\end{array}$ & $\begin{array}{c}\text { Water } \\
\text { temperature } \\
\left({ }^{\circ} \mathrm{C}\right)\end{array}$ & $\begin{array}{c}\text { Specific } \\
\text { conductance } \\
(\mu \mathrm{S} / \mathrm{cm})\end{array}$ & $\begin{array}{c}\text { pH } \\
\text { (standard } \\
\text { units) }\end{array}$ & $\begin{array}{c}\text { Dissolved } \\
\text { oxygen } \\
\text { (mg/L) }\end{array}$ & $\begin{array}{c}\text { Total } \\
\text { dissolved-gas } \\
\text { pressure } \\
(\mathrm{mm} \mathrm{Hg})\end{array}$ & $\begin{array}{c}\text { Dissolved or- } \\
\text { ganic carbon } \\
\text { (mg/L) }\end{array}$ & $\begin{array}{c}\text { Tritium } \\
\text { (TU) }\end{array}$ & $\begin{array}{l}\text { Tritium } \\
\text { precision } \\
\text { (TU) }\end{array}$ & $\begin{array}{c}\text { CFC-11 } \\
\text { (pmol/ } \\
\mathrm{kg})\end{array}$ & $\begin{array}{c}\text { CFC-12 } \\
\text { (pmol/ } \\
\text { kg) }\end{array}$ & $\begin{array}{l}\text { CFC-113 } \\
\text { (pmol/ } \\
\text { kg) }\end{array}$ & $\begin{array}{c}\mathrm{SF}_{6} \\
\text { (fmol/ } \\
\mathbf{k g})\end{array}$ \\
\hline \multicolumn{14}{|c|}{ Groundwater } \\
\hline \multirow[t]{16}{*}{ North Dam 3A } & $10 / 8 / 02$ & 15.9 & 4,430 & 8.0 & 5.0 & - & - & 2.71 & 0.14 & - & - & - & - \\
\hline & $12 / 18 / 02$ & 14.7 & 2,830 & 8.0 & 10.8 & - & - & - & - & - & - & - & - \\
\hline & $6 / 10 / 03$ & 21.5 & 1,330 & 7.8 & - & - & - & - & - & - & - & - & - \\
\hline & $10 / 9 / 03$ & - & 1,230 & 7.8 & - & - & - & - & - & - & - & - & - \\
\hline & $1 / 8 / 04$ & 16.0 & 1,220 & 8.2 & - & - & - & - & - & - & - & - & - \\
\hline & $9 / 21 / 04$ & 18.4 & 980 & 7.7 & 11.0 & - & - & - & - & - & - & - & - \\
\hline & $10 / 29 / 04$ & 15.9 & 910 & 7.9 & 11.1 & - & - & - & - & - & - & - & - \\
\hline & $2 / 10 / 05$ & 15.3 & 960 & 7.7 & 13.5 & - & - & - & - & - & - & - & - \\
\hline & $4 / 5 / 05$ & 16.5 & 960 & 7.8 & 12.6 & - & - & - & - & - & - & - & - \\
\hline & $1 / 19 / 06$ & - & 840 & 8.0 & - & - & - & - & - & - & - & - & - \\
\hline & $2 / 15 / 07$ & 15.2 & 840 & 7.9 & 7.5 & - & - & 2.53 & 0.31 & - & - & - & - \\
\hline & $3 / 14 / 08$ & 14.8 & 820 & 7.7 & 4.0 & - & - & 3.45 & 0.44 & - & - & - & - \\
\hline & 4/30/09 & - & 850 & 7.2 & - & - & - & 3.03 & 0.11 & - & - & - & - \\
\hline & $3 / 16 / 10$ & 22.8 & 860 & 7.6 & 1.3 & - & 1.91 & 3.05 & 0.12 & 0.54 & 2.0 & 0.10 & - \\
\hline & $3 / 10 / 11$ & 20.3 & 830 & 7.4 & 0.8 & - & 1.93 & 2.87 & 0.12 & 0.60 & 1.93 & 0.09 & - \\
\hline & $2 / 6 / 12$ & 11.3 & 820 & 7.8 & - & - & 2.00 & 3.46 & 0.41 & - & - & - & - \\
\hline
\end{tabular}


Table 2. Field measurements, total dissolved-gas pressure, and concentrations of dissolved organic carbon, tritium, chlorofluorocarbons, and sulfur hexafluoride in groundwater and surface water from Sand Hollow, Washington County, Utah.-Continued

[Analyzing agency: Dissolved organic carbon at U.S. Geological Survey (USGS) National Water Quality Laboratory in Denver, Colorado; Tritium at University of Utah Dissolved Gas Laboratory in Salt Lake City, Utah; CFC-11, CFC-12, CFC-113, and SF 6 at USGS Chlorofluorocarbon (CFC) Laboratory in Reston, Virginia. Abbreviations: ${ }^{\circ} \mathrm{C}$, degrees Celsius; $\mu \mathrm{S} / \mathrm{cm}$, microsiemens per centimeter at 25 degrees Celsius; $\mathrm{mg} / \mathrm{L}$, milligrams per liter; $\mathrm{mm} \mathrm{Hg}$, millimeters of mercury; TU, tritium units; pmol/kg, picomoles per kilogram; fmol/kg, femtomoles per kilogram; - , no data available; E, estimated; >, greater than; <, less than]

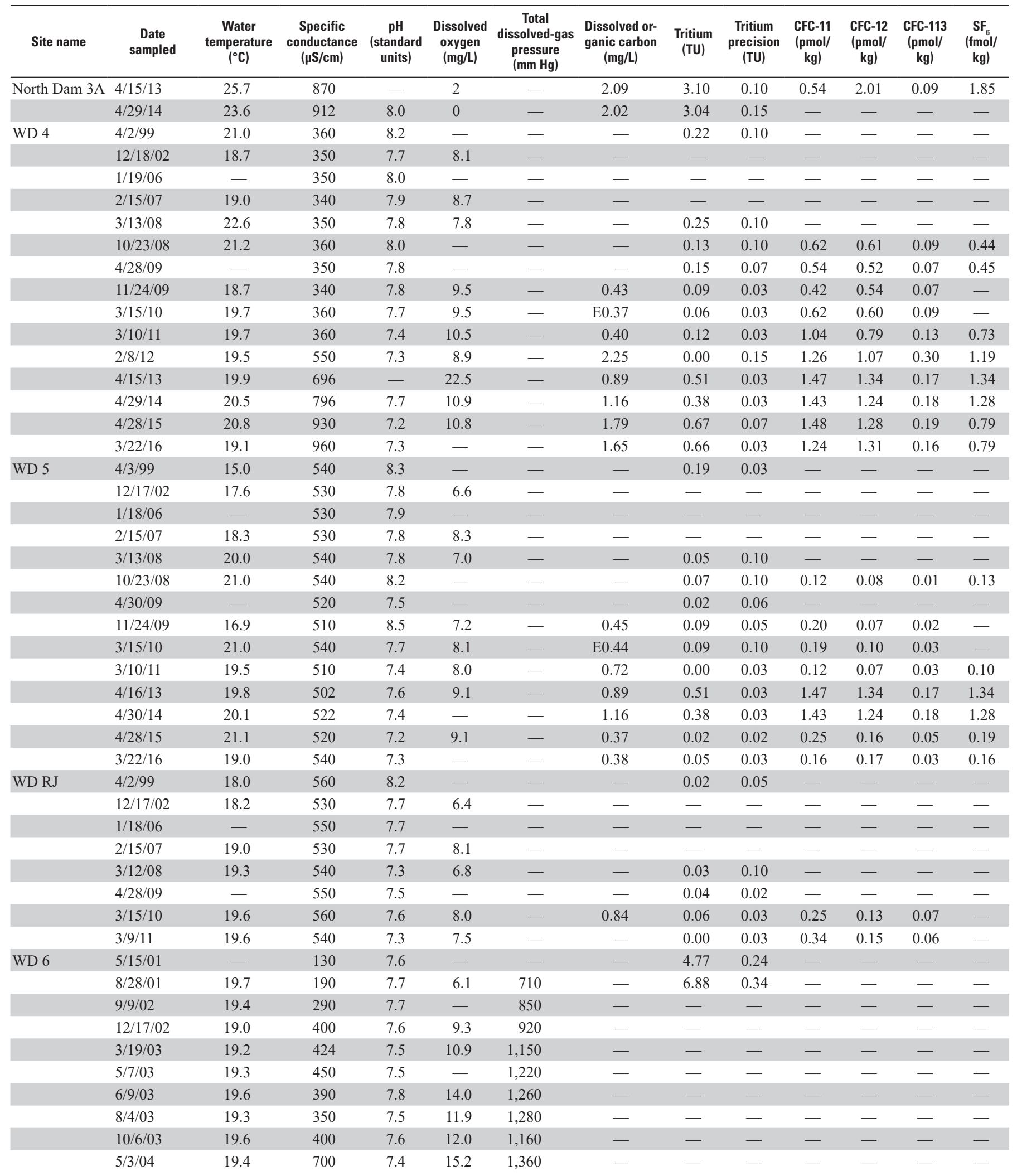


Table 2. Field water-quality parameters, total dissolved-gas pressure, dissolved organic carbon, tritium, chlorofluorocarbons, and sulfur hexafluoride in groundwater and surface water from Sand Hollow, Washington County, Utah.-Continued

[Analyzing agency: Dissolved organic carbon at U.S. Geological Survey (USGS) National Water Quality Laboratory in Denver, Colorado; Tritium at University of Utah Dissolved Gas Laboratory in Salt Lake City, Utah; CFC-11, CFC-12, CFC-113, and $\mathrm{SF}_{6}$ at USGS Chlorofluorocarbon (CFC) Laboratory in Reston, Virginia. Abbreviations: ${ }^{\circ} \mathrm{C}$, degrees Celsius; $\mu \mathrm{S} / \mathrm{cm}$, microsiemens per centimeter at 25 degrees Celsius; $\mathrm{mg} / \mathrm{L}$, milligrams per liter; mm Hg, millimeters of mercury; TU, tritium units; pmol/kg, picomoles per kilogram;

fmol/kg, femtomoles per kilogram; - , no data available; E, estimated; >, greater than; <, less than]

\begin{tabular}{|c|c|c|c|c|c|c|c|c|c|c|c|c|c|}
\hline Site name & $\begin{array}{c}\text { Date } \\
\text { sampled }\end{array}$ & $\begin{array}{c}\text { Water } \\
\text { temperature } \\
\left({ }^{\circ} \mathrm{C}\right)\end{array}$ & $\begin{array}{c}\text { Specific } \\
\text { conductance } \\
(\mu \mathrm{S} / \mathrm{cm})\end{array}$ & $\begin{array}{c}\mathrm{pH} \\
\text { (standard } \\
\text { units) }\end{array}$ & $\begin{array}{l}\text { Dissolved } \\
\text { oxygen } \\
\text { (mg/L) }\end{array}$ & $\begin{array}{c}\text { Total } \\
\text { dissolved-gas } \\
\text { pressure } \\
(\mathrm{mm} \mathrm{Hg})\end{array}$ & $\begin{array}{l}\text { Dissolved or- } \\
\text { ganic carbon } \\
\quad(\mathrm{mg} / \mathrm{L})\end{array}$ & $\begin{array}{c}\text { Tritium } \\
\text { (TU) }\end{array}$ & $\begin{array}{l}\text { Tritium } \\
\text { precision } \\
\text { (TU) }\end{array}$ & $\begin{array}{c}\text { CFC-11 } \\
\text { (pmol/ } \\
\text { kg) }\end{array}$ & $\begin{array}{c}\text { CFC-12 } \\
\text { (pmol/ } \\
\text { kg) }\end{array}$ & $\begin{array}{c}\text { CFC-113 } \\
\text { (pmol/ } \\
\mathbf{k g})\end{array}$ & $\begin{array}{c}\mathrm{SF}_{6} \\
\text { (fmol/ } \\
\mathbf{k g})\end{array}$ \\
\hline \multirow[t]{9}{*}{ WD 6} & $9 / 20 / 04$ & 19.6 & 820 & 7.7 & 15.0 & 1,270 & - & - & - & - & - & - & - \\
\hline & $10 / 28 / 04$ & 19.0 & 810 & 7.6 & 13.5 & 1,240 & - & - & - & - & - & - & - \\
\hline & $2 / 9 / 05$ & 19.2 & 450 & 7.9 & 14.6 & 1,460 & - & - & - & - & - & - & - \\
\hline & $4 / 5 / 05$ & 19.2 & 460 & 7.6 & 15.5 & 1,490 & - & - & - & - & - & - & - \\
\hline & $3 / 13 / 08$ & 19.2 & 1,300 & 7.5 & 14.4 & ${ }^{1} 1,590$ & - & 2.11 & 0.14 & - & - & - & - \\
\hline & $4 / 29 / 08$ & 19.3 & 1,290 & 7.7 & 17.1 & ${ }^{1} 1,590$ & - & - & - & - & - & - & - \\
\hline & $6 / 3 / 08$ & 19.4 & 1,330 & 7.6 & 16.5 & ${ }^{1} 1,590$ & - & - & - & - & - & - & - \\
\hline & $10 / 24 / 08$ & 19.0 & 1,190 & - & 16.3 & ${ }^{1} 1,540$ & - & 2.55 & 0.13 & 2.8 & 1.3 & 0.15 & 0.72 \\
\hline & 4/30/09 & 19.2 & 1,050 & 7.7 & 22.0 & ${ }^{1} 1,810$ & - & 2.66 & 0.14 & 3.2 & 1.5 & 0.16 & 0.73 \\
\hline \multirow{5}{*}{ (replicate) } & $2 / 7 / 12$ & 19.1 & 810 & 7.3 & 15.6 & ${ }^{1} 1,700$ & 1.70 & 2.88 & 0.31 & 2.23 & 1.67 & 0.18 & 0.87 \\
\hline & $4 / 16 / 13$ & 18.9 & 810 & 7.0 & 11.8 & ${ }^{1} 1,600$ & 1.18 & 2.52 & 0.14 & 2.1 & 1.9 & 0.19 & 0.71 \\
\hline & $4 / 29 / 14$ & 19.0 & 810 & 7.3 & 10.1 & ${ }^{1} 1,550$ & 0.92 & 2.85 & 0.11 & 2.0 & 1.9 & 0.19 & 0.87 \\
\hline & $4 / 28 / 15$ & 19.1 & 820 & 7.6 & 14.7 & ${ }^{1} 1,630$ & 0.98 & 2.76 & 0.11 & 2.1 & 1.8 & 0.19 & 0.70 \\
\hline & $3 / 22 / 16$ & 18.8 & 850 & 7.7 & 13.2 & ${ }^{1} 1,610$ & 0.78 & 2.55 & 0.15 & 1.6 & 1.6 & 0.12 & 0.58 \\
\hline \multirow[t]{6}{*}{ WD 8} & $5 / 21 / 01$ & - & 300 & 7.7 & - & - & - & 4.13 & 0.38 & - & - & - & - \\
\hline & 9/12/01 & 18.7 & 305 & 7.7 & 9.6 & 890 & - & 2.98 & 0.15 & - & - & - & - \\
\hline & $9 / 9 / 02$ & 18.9 & 305 & 7.9 & - & 840 & - & 3.89 & 0.19 & - & - & - & - \\
\hline & $3 / 20 / 03$ & 18.7 & 335 & 7.6 & 7.8 & 910 & - & - & - & - & - & - & - \\
\hline & $5 / 8 / 03$ & 18.6 & 340 & 7.5 & 4.6 & 880 & - & - & - & - & - & - & - \\
\hline & $10 / 16 / 03$ & - & 360 & 7.4 & - & - & - & - & - & - & - & - & - \\
\hline \multirow{15}{*}{ WD 9} & 9/11/02 & 19.5 & 350 & 7.9 & - & 980 & - & - & - & - & - & - & - \\
\hline & $5 / 7 / 03$ & 19.7 & 320 & 7.8 & - & ${ }^{1}>2,250$ & - & - & - & - & - & - & - \\
\hline & $6 / 9 / 03$ & 19.5 & 350 & 7.7 & 24.4 & ${ }^{1}>2,250$ & - & - & - & - & - & - & - \\
\hline & $8 / 5 / 03$ & 19.7 & 720 & 7.5 & 19.3 & ${ }^{1} 1,800$ & - & - & - & - & - & - & - \\
\hline & $10 / 7 / 03$ & 19.6 & 740 & 7.5 & 17.9 & ${ }^{1} 1,600$ & - & - & - & - & - & - & - \\
\hline & $1 / 6 / 04$ & 19.4 & 630 & 7.7 & 16.7 & ${ }^{1} 1,700$ & - & - & - & - & - & - & - \\
\hline & $5 / 3 / 04$ & 19.4 & 530 & 7.4 & 25.7 & ${ }^{1}>2,250$ & - & - & - & - & - & - & - \\
\hline & $9 / 20 / 04$ & 18.5 & 750 & 7.8 & 22.6 & ${ }^{1}>2,250$ & - & - & - & - & - & - & - \\
\hline & $10 / 28 / 04$ & 18.5 & 760 & 7.6 & 20.7 & ${ }^{1} 2,210$ & - & - & - & - & - & - & - \\
\hline & $2 / 9 / 05$ & 18.4 & 880 & 7.7 & 20.2 & ${ }^{1}>2,250$ & - & - & - & - & - & - & - \\
\hline & $4 / 5 / 05$ & 18.5 & 820 & 7.4 & 23.2 & ${ }^{1>2,250}$ & - & - & - & - & - & - & - \\
\hline & $1 / 18 / 06$ & 18.0 & 1,230 & 7.9 & 15.0 & ${ }^{1} 1,900$ & - & - & - & - & - & - & - \\
\hline & $2 / 14 / 07$ & 17.3 & 790 & 7.4 & 4.6 & ${ }^{1} 1,600$ & - & - & - & - & - & - & - \\
\hline & $3 / 11 / 08$ & 17.0 & 820 & 7.3 & 1.5 & 1,080 & - & 2.61 & 0.22 & - & - & - & - \\
\hline & $4 / 27 / 09$ & 16.6 & 830 & 7.4 & 1.8 & 840 & - & 2.99 & 0.12 & 1.2 & 2.2 & 0.19 & 2.15 \\
\hline
\end{tabular}


Table 2. Field water-quality parameters, total dissolved-gas pressure, dissolved organic carbon, tritium, chlorofluorocarbons, and sulfur hexafluoride in groundwater and surface water from Sand Hollow, Washington County, Utah.-Continued

[Analyzing agency: Dissolved organic carbon at U.S. Geological Survey (USGS) National Water Quality Laboratory in Denver, Colorado; Tritium at University of Utah Dissolved Gas Laboratory in Salt Lake City, Utah; CFC-11, CFC-12, CFC-113, and $\mathrm{SF}_{6}$ at USGS Chlorofluorocarbon (CFC) Laboratory in Reston, Virginia. Abbreviations: ${ }^{\circ} \mathrm{C}$, degrees Celsius; $\mu \mathrm{S} / \mathrm{cm}$, microsiemens per centimeter at 25 degrees Celsius; $\mathrm{mg} / \mathrm{L}$, milligrams per liter; $\mathrm{mm} \mathrm{Hg}$, millimeters of mercury; TU, tritium units; pmol/kg, picomoles per kilogram; fmol $/ \mathrm{kg}$, femtomoles per kilogram; - , no data available; E, estimated; >, greater than; <, less than]

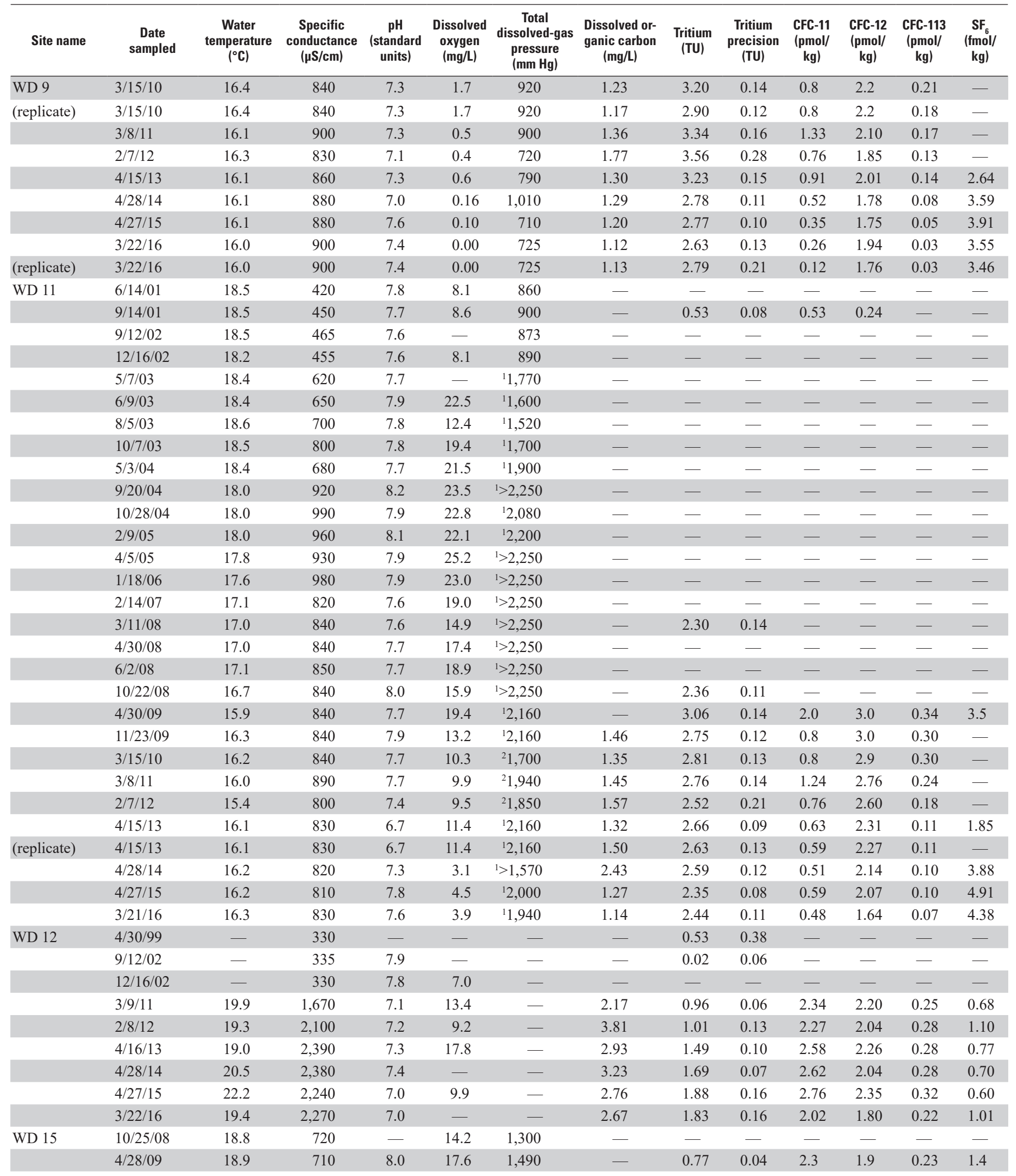


Table 2. Field water-quality parameters, total dissolved-gas pressure, dissolved organic carbon, tritium, chlorofluorocarbons, and sulfur hexafluoride in groundwater and surface water from Sand Hollow, Washington County, Utah.-Continued

[Analyzing agency: Dissolved organic carbon at U.S. Geological Survey (USGS) National Water Quality Laboratory in Denver, Colorado; Tritium at University of Utah Dissolved Gas Laboratory in Salt Lake City, Utah; CFC-11, CFC-12, CFC-113, and SF at USGS Chlorofluorocarbon (CFC) Laboratory in Reston, Virginia. Abbreviations: ${ }^{\circ} \mathrm{C}$, degrees Celsius; $\mu \mathrm{S} / \mathrm{cm}$, microsiemens per centimeter at 25 degrees Celsius; $\mathrm{mg} / \mathrm{L}$, milligrams per liter; $\mathrm{mm} \mathrm{Hg}$, millimeters of mercury; TU, tritium units; pmol/kg, picomoles per kilogram; fmol/kg, femtomoles per kilogram; - , no data available; E, estimated; >, greater than; <, less than]

\begin{tabular}{|c|c|c|c|c|c|c|c|c|c|c|c|c|c|}
\hline Site name & $\begin{array}{l}\text { Date } \\
\text { sampled }\end{array}$ & $\begin{array}{c}\text { Water } \\
\text { temperature } \\
\left({ }^{\circ} \mathrm{C}\right)\end{array}$ & $\begin{array}{c}\text { Specific } \\
\text { conductance } \\
(\mu \mathrm{S} / \mathrm{cm})\end{array}$ & $\begin{array}{c}\mathrm{pH} \\
\text { (standard } \\
\text { units) }\end{array}$ & $\begin{array}{c}\text { Dissolved } \\
\text { oxygen } \\
\text { (mg/L) }\end{array}$ & $\begin{array}{c}\text { Total } \\
\text { dissolved-gas } \\
\text { pressure } \\
(\mathrm{mm} \mathrm{Hg})\end{array}$ & $\begin{array}{l}\text { Dissolved or- } \\
\text { ganic carbon } \\
\text { (mg/L) }\end{array}$ & $\begin{array}{c}\text { Tritium } \\
\text { (TU) }\end{array}$ & $\begin{array}{l}\text { Tritium } \\
\text { precision } \\
\text { (TU) }\end{array}$ & $\begin{array}{c}\text { CFC-11 } \\
\text { (pmol/ } \\
\text { kg) }\end{array}$ & $\begin{array}{c}\text { CFC-12 } \\
\text { (pmol/ } \\
\mathrm{kg})\end{array}$ & $\begin{array}{c}\text { CFC-113 } \\
\text { (pmol/ } \\
\text { kg) }\end{array}$ & $\begin{array}{c}\mathrm{SF}_{6} \\
(\mathrm{fmol} / \\
\mathrm{kg})\end{array}$ \\
\hline \multirow[t]{3}{*}{ WD 15} & $11 / 23 / 09$ & 18.8 & 730 & 8.3 & 14.5 & 1,410 & 2.47 & 0.68 & 0.05 & 1.0 & 1.9 & 0.22 & - \\
\hline & $3 / 8 / 11$ & 19.1 & 820 & 8.0 & 12.5 & 1,400 & 2.50 & 0.55 & 0.06 & 2.56 & 2.27 & 0.28 & 1.56 \\
\hline & $2 / 7 / 12$ & 19.1 & 820 & 8.0 & 18.8 & ${ }^{2} 1,450$ & 3.15 & 0.15 & 0.13 & 2.36 & 2.14 & 0.27 & 1.48 \\
\hline \multirow[t]{2}{*}{ (replicate) } & $4 / 29 / 14$ & 18.8 & 970 & 7.7 & 11.7 & ${ }^{1} 1,560$ & 2.72 & 0.50 & 0.04 & 2.14 & 2.20 & 0.27 & 1.25 \\
\hline & $4 / 29 / 15$ & 18.8 & 1,090 & 8.0 & 19.1 & ${ }^{1} 1,730$ & 2.71 & 0.51 & 0.05 & 2.26 & 1.84 & 0.23 & 0.99 \\
\hline \multirow[t]{2}{*}{ (replicate) } & $4 / 29 / 15$ & 18.8 & 1,090 & 8.0 & 19.1 & ${ }^{1} 1,730$ & 2.73 & 0.47 & 0.09 & 2.34 & 1.85 & 0.24 & 1.00 \\
\hline & $3 / 23 / 16$ & 19.0 & 1,130 & 8.0 & 18.9 & ${ }^{1} 1,680$ & 2.37 & 0.51 & 0.04 & 1.84 & 1.63 & 0.16 & 0.93 \\
\hline \multirow[t]{5}{*}{ WD 16} & $10 / 25 / 08$ & 18.7 & 470 & 8.0 & 7.7 & 780 & - & - & - & - & - & - & - \\
\hline & $4 / 17 / 13$ & 18.7 & 430 & - & 8.1 & 830 & $<0.23$ & 0.05 & 0.02 & 0.11 & 0.05 & 0.01 & 1.16 \\
\hline & $4 / 29 / 14$ & 18.7 & 450 & 7.4 & 3.7 & 1,060 & $<0.23$ & -0.05 & -0.15 & 0.20 & 0.15 & 0.01 & 0.27 \\
\hline & $4 / 29 / 15$ & 19.0 & 450 & 7.6 & 7.2 & 760 & $<0.23$ & 0.04 & 0.01 & 0.06 & 0.04 & 0.01 & 0.23 \\
\hline & $3 / 23 / 16$ & 18.5 & 450 & 7.8 & 8.0 & 760 & $<0.23$ & 0.03 & 0.03 & 0.06 & 0.00 & 0.01 & 0.16 \\
\hline \multirow[t]{3}{*}{ WD 17} & $10 / 24 / 08$ & 19.5 & 615 & - & 8.4 & 1,000 & - & - & - & - & - & - & - \\
\hline & $4 / 16 / 13$ & 19.4 & 545 & 7.6 & 11.0 & 1,030 & 0.54 & 0.05 & 0.01 & 0.79 & 0.76 & 0.09 & 2.29 \\
\hline & $3 / 23 / 16$ & 19.5 & 500 & 7.3 & 12.5 & 1,120 & 0.54 & 0.02 & 0.03 & 1.37 & 1.02 & 0.12 & 0.78 \\
\hline \multirow[t]{4}{*}{ WD 18} & $4 / 28 / 09$ & 19.7 & 500 & 7.4 & 7.5 & 870 & - & 0.04 & 0.02 & 0.21 & 0.16 & 0.03 & 1.46 \\
\hline & $3 / 16 / 10$ & 19.3 & 470 & 7.4 & 4.9 & 740 & E0.48 & 0.14 & 0.02 & 0.16 & 0.07 & 0.01 & - \\
\hline & $3 / 9 / 11$ & 19.2 & 480 & 7.3 & 7.8 & 750 & 0.70 & 0.03 & 0.03 & 0.08 & 0.02 & 0.02 & - \\
\hline & $3 / 23 / 16$ & 19.5 & 490 & 7.5 & 8.1 & 780 & 0.35 & 0.04 & 0.02 & 0.20 & 0.23 & 0.04 & 0.46 \\
\hline \multirow{3}{*}{ (replicate) } & $3 / 17 / 10$ & 19.4 & 340 & 7.4 & 7.2 & 720 & $<0.66$ & 0.03 & 0.04 & 0.11 & 0.04 & 0.01 & - \\
\hline & $3 / 10 / 11$ & 18.9 & 330 & 7.3 & 7.2 & 710 & 0.23 & 0.01 & 0.04 & 0.15 & 0.07 & 0.02 & - \\
\hline & $3 / 23 / 16$ & 19.4 & 330 & 7.2 & 9.8 & 700 & $<0.23$ & 0.00 & 0.03 & 0.27 & 0.25 & 0.04 & 0.32 \\
\hline WD 21 & $3 / 22 / 16$ & 19.1 & 420 & 7.9 & 6.5 & 740 & 0.76 & 0.11 & 0.04 & 0.32 & 1.07 & 0.08 & 0.68 \\
\hline \multirow[t]{2}{*}{ WD 22} & $9 / 18 / 15$ & 19.3 & 680 & 7.6 & 17.9 & ${ }^{1} 1,730$ & 0.75 & 1.38 & 0.17 & 1.13 & 0.69 & 0.05 & 0.31 \\
\hline & $3 / 23 / 16$ & 19.1 & 730 & 7.4 & 18.9 & ${ }^{1} 1,820$ & 0.74 & 1.43 & 0.07 & 1.76 & 1.16 & 0.1 & 0.32 \\
\hline
\end{tabular}


Table 2. Field water-quality parameters, total dissolved-gas pressure, dissolved organic carbon, tritium, chlorofluorocarbons, and sulfur hexafluoride in groundwater and surface water from Sand Hollow, Washington County, Utah.-Continued

[Analyzing agency: Dissolved organic carbon at U.S. Geological Survey (USGS) National Water Quality Laboratory in Denver, Colorado; Tritium at University of Utah Dissolved Gas Laboratory in Salt Lake City, Utah; CFC-11, CFC-12, CFC-113, and SF at USGS Chlorofluorocarbon (CFC) Laboratory in Reston, Virginia. Abbreviations: ${ }^{\circ}$ C, degrees Celsius; $\mu \mathrm{S} / \mathrm{cm}$, microsiemens per centimeter at 25 degrees Celsius; $\mathrm{mg} / \mathrm{L}$, milligrams per liter; $\mathrm{mm} \mathrm{Hg}$, millimeters of mercury; $\mathrm{TU}$, tritium units; pmol/kg, picomoles per kilogram; $\mathrm{fmol} / \mathrm{kg}$, femtomoles per kilogram; - , no data available; E, estimated; >, greater than; <, less than]

\begin{tabular}{|c|c|c|c|c|c|c|c|c|c|c|c|c|c|}
\hline Site name & $\begin{array}{c}\text { Date } \\
\text { sampled }\end{array}$ & $\begin{array}{c}\text { Water } \\
\text { temperature } \\
\left({ }^{\circ} \mathrm{C}\right)\end{array}$ & $\begin{array}{c}\text { Specific } \\
\text { conductance } \\
(\mu \mathrm{S} / \mathrm{cm})\end{array}$ & $\begin{array}{c}\mathrm{pH} \\
\text { (standard } \\
\text { units) }\end{array}$ & $\begin{array}{c}\text { Dissolved } \\
\text { oxygen } \\
\text { (mg/L) }\end{array}$ & $\begin{array}{c}\text { Total } \\
\text { dissolved-gas } \\
\text { pressure } \\
(\mathrm{mm} \mathrm{Hg})\end{array}$ & $\begin{array}{l}\text { Dissolved or- } \\
\text { ganic carbon } \\
\quad(\mathrm{mg} / \mathrm{L})\end{array}$ & $\begin{array}{l}\text { Tritium } \\
\text { (TU) }\end{array}$ & $\begin{array}{l}\text { Tritium } \\
\text { precision } \\
\text { (TU) }\end{array}$ & $\begin{array}{c}\text { CFC-11 } \\
\text { (pmol/ } \\
\text { kg) }\end{array}$ & $\begin{array}{c}\text { CFC-12 } \\
\text { (pmol/ } \\
\text { kg) }\end{array}$ & $\begin{array}{c}\text { CFC-113 } \\
(\mathrm{pmol} / \\
\mathrm{kg})\end{array}$ & $\begin{array}{c}\mathrm{SF}_{6} \\
(\mathrm{fmol} / \\
\mathbf{k g})\end{array}$ \\
\hline \multicolumn{14}{|c|}{ Sand Hollow Reservoir water } \\
\hline \multirow[t]{11}{*}{ Boat ramp } & $5 / 5 / 04$ & 17.3 & 710 & 8.2 & 8.5 & 680 & - & - & - & - & - & - & - \\
\hline & $9 / 22 / 04$ & 18.9 & 770 & 8.5 & 7.2 & - & - & - & - & - & - & - & - \\
\hline & $2 / 10 / 05$ & 8.3 & 860 & 8.4 & 11.3 & - & - & - & - & - & - & - & - \\
\hline & $3 / 13 / 08$ & 9.6 & 820 & 8.4 & 10.1 & - & - & - & - & - & - & - & - \\
\hline & $10 / 21 / 08$ & 18.3 & 820 & 8.7 & 8.9 & 700 & - & 3.59 & 0.18 & 2.3 & 1.5 & 0.22 & 1.49 \\
\hline & $4 / 29 / 09$ & 16.1 & 790 & 8.4 & 7.0 & - & - & 4.61 & 0.20 & 3.1 & 2.0 & 0.32 & 1.94 \\
\hline & $8 / 10 / 09$ & 25.0 & 800 & 8.6 & - & - & 2.85 & - & - & - & - & - & - \\
\hline & $11 / 24 / 09$ & 11.3 & 800 & 8.5 & 9.5 & - & 2.95 & 3.29 & 0.14 & 2.1 & 2.6 & 0.30 & - \\
\hline & $4 / 29 / 14$ & 15.2 & 850 & 9.0 & 10.5 & 690 & 2.64 & 2.61 & 0.10 & 3.36 & 2.18 & 0.31 & 2.00 \\
\hline & $4 / 27 / 15$ & 16.5 & 840 & 8.0 & 9.0 & 670 & 2.68 & 2.31 & 0.08 & 3.44 & 2.25 & 0.31 & 2.00 \\
\hline & $3 / 23 / 16$ & 12.9 & 830 & 8.2 & 9.8 & 710 & 2.07 & 2.52 & 0.17 & 3.58 & 2.23 & 0.31 & 1.74 \\
\hline \multirow[t]{8}{*}{ SH1-18 } & $10 / 23 / 08$ & 18.0 & 820 & 8.7 & 9.1 & 690 & - & 4.60 & 0.34 & 2.5 & 1.6 & 0.23 & 1.16 \\
\hline & $4 / 29 / 09$ & 14.3 & 800 & 8.6 & 9.6 & - & - & 2.55 & 0.22 & 3.4 & 2.1 & 0.26 & 1.98 \\
\hline & $8 / 10 / 09$ & 25.3 & 800 & 8.7 & 9.1 & - & 5.67 & - & - & - & - & - & - \\
\hline & $3 / 16 / 10$ & 9.6 & 820 & 8.0 & 9.6 & - & 2.87 & 3.68 & 0.13 & 3.0 & 3.2 & 0.44 & - \\
\hline & $3 / 9 / 11$ & 8.1 & 820 & 8.2 & 10.7 & 700 & 2.66 & 3.52 & 0.13 & 5.47 & 3.19 & 0.50 & - \\
\hline & $2 / 8 / 12$ & 5.9 & 800 & 8.0 & 10.7 & 670 & 2.76 & 3.49 & 0.53 & 5.45 & 3.05 & 0.52 & - \\
\hline & $4 / 18 / 13$ & 12.5 & 880 & 8.0 & 8.7 & 680 & 2.55 & 3.26 & 0.12 & 3.92 & 2.43 & 0.31 & 2.39 \\
\hline & $4 / 30 / 14$ & 14.1 & 840 & 8.2 & - & 700 & 2.78 & 2.98 & 0.16 & - & - & - & - \\
\hline
\end{tabular}

${ }^{1}$ Total dissolved-gas pressures greater than $1,500 \mathrm{~mm} \mathrm{Hg}$ exceed the linear calibration of the multi-parameter sonde.

${ }^{2}$ Total dissolved-gas pressure determined with advanced diffusion sampler.

${ }^{3}$ WD RJ monitoring well removed in 2013. 
Table 3. Field measurements and concentrations of major ions, selected trace elements, and nutrients in groundwater and surface water from selected sites in Sand Hollow, Washington County, Utah.

$\left[{ }^{\circ} \mathrm{C}\right.$, degrees Celsius; $\mu \mathrm{S} / \mathrm{cm}$, microsiemens per centimeter at 25 degrees Celsius; $\mathrm{mg} / \mathrm{L}$, milligrams per liter; $\mathrm{Cl} / \mathrm{Br}$, chloride to bromide ratio; $\mu \mathrm{g} / \mathrm{L}$, micrograms per liter; $\mu \mathrm{g} / \mathrm{L}$, micrograms per liter; - , no data available; $<$, less than; E, estimated; ft, feet]

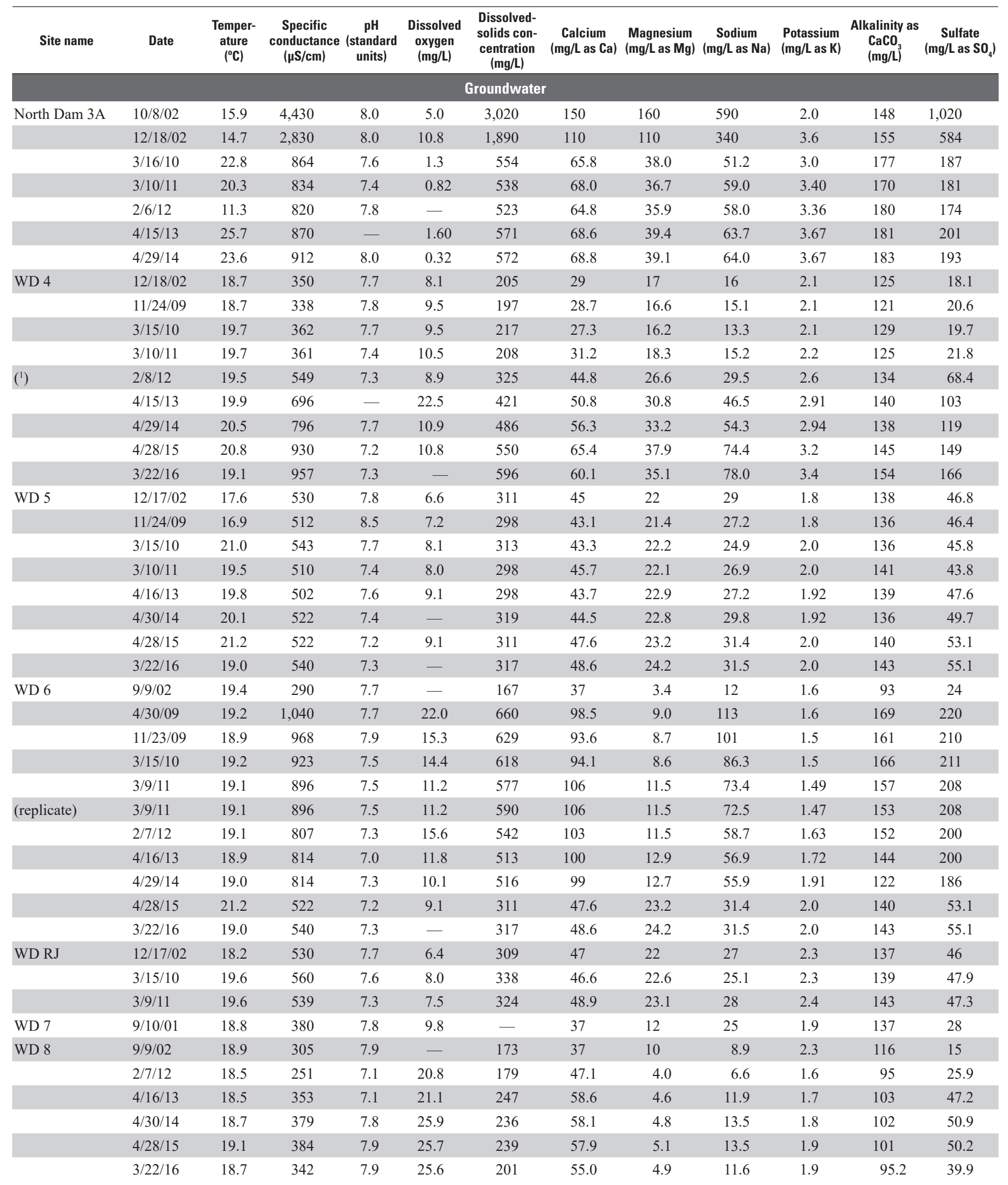


Table 3. Field measurements and concentrations of major ions, selected trace elements, and nutrients in groundwater and surface water from selected sites in Sand Hollow, Washington County, Utah.-Continued

$\left[{ }^{\circ} \mathrm{C}\right.$, degrees Celsius; $\mu \mathrm{S} / \mathrm{cm}$, microsiemens per centimeter at 25 degrees Celsius; mg/L, milligrams per liter; $\mathrm{Cl} / \mathrm{Br}$, chloride to bromide ratio; $\mu \mathrm{g} / \mathrm{L}$, micrograms per liter; $\mu \mathrm{g} / \mathrm{L}$, micrograms per liter; - , no data available; <, less than; E, estimated; ft, feet]

\begin{tabular}{|c|c|c|c|c|c|c|c|c|c|c|c|c|}
\hline Site name & $\begin{array}{l}\text { Chloride } \\
\text { (mg/L as Cl) }\end{array}$ & $\begin{array}{l}\text { Fluoride } \\
\text { (mg/L as F) }\end{array}$ & $\begin{array}{c}\text { Bromide } \\
(\mathrm{mg} / \mathrm{L} \text { as } \mathrm{Br})\end{array}$ & $\mathrm{Cl} / \mathrm{Br}$ & $\begin{array}{c}\text { Silica } \\
\text { (mg/L as } \\
\left.\mathrm{SiO}_{2}\right)\end{array}$ & $\begin{array}{c}\text { Iron } \\
(\mu \mathrm{g} / \mathrm{L} \text { as } \mathrm{Fe})\end{array}$ & $\begin{array}{c}\text { Manganese } \\
(\mu \mathrm{g} / \mathrm{L} \text { as } \\
\mathrm{Mn})\end{array}$ & $\begin{array}{c}\text { Arsenic } \\
(\mu \mathrm{g} / \mathrm{L} \text { as As) }\end{array}$ & $\begin{array}{c}\text { Nitrogen } \\
\text { (nitrite + nitrate) } \\
(\mathrm{mg} / \mathrm{L} \text { as N) }\end{array}$ & $\begin{array}{c}\text { Nitrogen, } \\
\text { nitrite } \\
\text { (mg/L as N) }\end{array}$ & $\begin{array}{c}\text { Nitrogen, } \\
\text { ammonia } \\
\text { (mg/L as N) }\end{array}$ & $\begin{array}{c}\text { Phosphorus } \\
\text { (orthophos- } \\
\text { phate) } \\
\text { (mg/L as P) }\end{array}$ \\
\hline \multicolumn{13}{|c|}{ Groundwater } \\
\hline \multirow[t]{5}{*}{ North Dam 3A } & 744 & 0.90 & 41.2 & 18 & 13.0 & $<30$ & $<5$ & 90.1 & 17.8 & $<0.008$ & 0.03 & 0.030 \\
\hline & 476 & 0.79 & 2.44 & 195 & 14.0 & $<30$ & $<5$ & 63.9 & 14.3 & $<0.008$ & $<0.04$ & 0.030 \\
\hline & 55.2 & 0.43 & 0.06 & 882 & 15.7 & $<6$ & 3.19 & 35.2 & $<0.04$ & E0.001 & E0.01 & 0.035 \\
\hline & 60.4 & 0.34 & 0.08 & 715 & 15.0 & 4.2 & 26.1 & 35.6 & 0.04 & $<0.001$ & 0.31 & 0.038 \\
\hline & 60 & 0.37 & 0.06 & 938 & 15.0 & $<4$ & 21.2 & 34.5 & 0.04 & $<0.001$ & 0.38 & 0.038 \\
\hline \multirow[t]{2}{*}{ WD 4} & 18.8 & 0.23 & 0.08 & 235 & 14 & $<10$ & $<2$ & 13.2 & 2.35 & $<0.008$ & $<0.04$ & 0.02 \\
\hline & 17.2 & 0.23 & 0.10 & 179 & 14.3 & E3.8 & $<0.2$ & 14.7 & 2.29 & $<0.002$ & $<0.02$ & 0.03 \\
\hline \multirow{3}{*}{ (1) } & 93.6 & 0.21 & 0.63 & 149 & 14.5 & $<4.0$ & 0.26 & 11.8 & 2.22 & $<0.001$ & $<0.01$ & 0.022 \\
\hline & 112.0 & 0.21 & 0.77 & 145 & 15.3 & $<4.0$ & 0.22 & 11.6 & 2.12 & $<0.001$ & $<0.01$ & 0.026 \\
\hline & 116.0 & 0.26 & 0.76 & 153 & 16.4 & $<4.0$ & $<0.20$ & 12.1 & 2.10 & $<0.001$ & 0.01 & 0.025 \\
\hline \multirow[t]{7}{*}{ WD 5} & 44.8 & 0.29 & 0.16 & 280 & 13 & $<10$ & E1 & 9.1 & 4.18 & $<0.008$ & $<0.04$ & E0.01 \\
\hline & 37.9 & 0.27 & 0.23 & 168 & 13.4 & $<6$ & $<0.2$ & 9.6 & 4.61 & $<0.002$ & $<0.02$ & 0.02 \\
\hline & 39.2 & 0.28 & 0.24 & 164 & 15 & $<6$ & $<0.2$ & 9.0 & 4.60 & $<0.002$ & $<0.02$ & 0.01 \\
\hline & 38.2 & 0.31 & 0.21 & 179 & 14.3 & $<3.2$ & 0.19 & 9.3 & 4.60 & $<0.001$ & $<0.01$ & 0.01 \\
\hline & 40.3 & 0.24 & 0.22 & 187 & 13.6 & 4.8 & 0.41 & 9.2 & 4.73 & $<0.001$ & $<0.01$ & 0.012 \\
\hline & 42.8 & 0.26 & 0.23 & 189 & 13.7 & $<4.0$ & $<0.02$ & 9.4 & 4.73 & $<0.001$ & $<0.01$ & 0.010 \\
\hline & 45.8 & 0.24 & 0.25 & 185 & 14.4 & $<4.0$ & $<0.20$ & 8.9 & 4.70 & $<0.001$ & $<0.01$ & 0.013 \\
\hline \multirow[t]{6}{*}{ (replicate) } & 63.3 & 0.32 & 0.14 & 455 & 13.6 & 6.1 & 0.31 & 2.8 & 0.70 & 0.001 & $<0.01$ & 0.009 \\
\hline & 54.8 & 0.22 & 0.08 & 684 & 13.9 & 17.6 & 0.75 & 2.3 & 0.47 & $<0.001$ & 0.02 & 0.011 \\
\hline & 57.2 & 0.18 & 0.07 & 840 & 12.9 & 20.3 & 0.88 & 2.3 & 0.34 & $<0.001$ & $<0.01$ & 0.009 \\
\hline & 53.8 & 0.18 & 0.06 & 928 & 12.7 & 10.5 & 0.50 & 2.1 & 0.32 & $<0.001$ & $<0.01$ & 0.012 \\
\hline & 53.7 & 0.16 & 0.06 & 895 & 13.5 & 6.1 & 0.46 & 2.2 & 0.243 & $<0.001$ & $<0.01$ & 0.012 \\
\hline & 61.3 & 0.24 & 0.05 & 1,157 & 13.7 & 9.5 & 0.55 & 2.2 & 0.285 & $<0.001$ & $<0.01$ & 0.012 \\
\hline \multirow[t]{3}{*}{ WD RJ } & 47.8 & 0.51 & 0.20 & 239 & 14 & $<10$ & $<2$ & 7.9 & 3.28 & $<0.008$ & $<0.04$ & 0.01 \\
\hline & 47.2 & 0.51 & 0.27 & 176 & 15.3 & $<6$ & $<0.2$ & 8.3 & 3.28 & $<0.002$ & E0.01 & 0.02 \\
\hline & 47.4 & 0.54 & 0.24 & 196 & 14.5 & $<3.2$ & 0.18 & 8.5 & 3.34 & $<0.001$ & $<0.01$ & 0.01 \\
\hline WD 7 & 18.0 & 0.3 & 0.13 & 139 & 14 & $<10$ & $<3$ & 6.0 & 3.80 & $<0.008$ & $<0.04$ & 0.02 \\
\hline \multirow[t]{6}{*}{ WD 8} & 10.1 & 0.1 & 0.07 & 144 & 14 & $<10$ & $<2$ & 6.0 & 3.90 & $<0.008$ & $<0.04$ & 0.02 \\
\hline & 13.6 & 0.1 & 0.08 & 173 & 13.8 & 7.4 & 0.99 & 11.5 & 3.33 & $<0.001$ & $<0.01$ & 0.02 \\
\hline & 19.4 & 0.07 & 0.12 & 169 & 13.4 & 11.7 & 2.25 & 10.6 & 3.52 & $<0.001$ & $<0.01$ & 0.014 \\
\hline & 20.5 & 0.07 & 0.10 & 199 & 13.5 & 9.3 & 2.07 & 10.0 & 3.60 & $<0.001$ & $<0.01$ & 0.015 \\
\hline & 20.8 & 0.07 & 0.11 & 193 & 14.2 & 20.0 & 1.19 & 8.7 & 3.52 & $<0.001$ & $<0.01$ & 0.017 \\
\hline & 17.6 & 0.08 & 0.09 & 189 & 14.2 & 9.5 & 1.01 & 10.0 & 3.25 & 0.001 & 0.01 & 0.016 \\
\hline
\end{tabular}


Table 3. Field measurements and concentrations of major ions, selected trace elements, and nutrients in groundwater and surface water from selected sites in Sand Hollow, Washington County, Utah.-Continued

$\left[{ }^{\circ} \mathrm{C}\right.$, degrees Celsius; $\mu \mathrm{S} / \mathrm{cm}$, microsiemens per centimeter at 25 degrees Celsius; $\mathrm{mg} / \mathrm{L}$, milligrams per liter; $\mathrm{Cl} / \mathrm{Br}$, chloride to bromide ratio; $\mu \mathrm{g} / \mathrm{L}$, micrograms per liter; $\mu \mathrm{g} / \mathrm{L}$, micrograms per liter; - , no data available; <, less than; E, estimated; ft, feet]

\begin{tabular}{|c|c|c|c|c|c|c|c|c|c|c|c|c|}
\hline Site name & Date & $\begin{array}{l}\text { Temper- } \\
\text { ature } \\
\left({ }^{\circ} \mathrm{C}\right)\end{array}$ & $\begin{array}{c}\text { Specific } \\
\text { conductance } \\
(\mu \mathrm{S} / \mathrm{cm})\end{array}$ & $\begin{array}{c}\mathrm{pH} \\
\text { (standard } \\
\text { units) }\end{array}$ & $\begin{array}{c}\text { Dissolved } \\
\text { oxygen } \\
\text { (mg/L) }\end{array}$ & $\begin{array}{c}\text { Dissolved- } \\
\text { solids con- } \\
\text { centration } \\
(\mathrm{mg} / \mathrm{L})\end{array}$ & $\begin{array}{l}\text { Calcium } \\
\text { (mg/L as Ca) }\end{array}$ & $\begin{array}{l}\text { Magnesium } \\
\text { (mg/L as Mg) }\end{array}$ & $\begin{array}{c}\text { Sodium } \\
\text { (mg/L as } \mathrm{Na})\end{array}$ & $\begin{array}{l}\text { Potassium } \\
\text { (mg/L as K) }\end{array}$ & $\begin{array}{c}\text { Alkalinity as } \\
\mathrm{CaCO}_{3} \\
(\mathrm{mg} / \mathrm{L})\end{array}$ & $\begin{array}{c}\text { Sulfate } \\
\left(\mathrm{mg} / \mathrm{L} \text { as } \mathrm{SO}_{4}\right)\end{array}$ \\
\hline \multirow[t]{2}{*}{ WD 9} & $9 / 11 / 02$ & 19.5 & 335 & 7.9 & - & 189 & 36 & 7 & 22 & 1.6 & 120 & 18 \\
\hline & $3 / 15 / 10$ & 16.4 & 842 & 7.3 & 1.7 & 543 & 71.8 & 31.0 & 52.2 & 3.3 & 157 & 200 \\
\hline \multirow[t]{5}{*}{ (replicate) } & $3 / 15 / 10$ & 16.4 & 842 & 7.3 & 1.7 & 545 & 67.4 & 28.8 & 51.0 & 3.2 & 155 & 198 \\
\hline & $4 / 15 / 13$ & 16.1 & 855 & 7.3 & 0.60 & 545 & 67.0 & 38.4 & 62.1 & 3.73 & 167 & 207 \\
\hline & $4 / 28 / 14$ & 16.1 & 877 & 7.0 & 0.16 & 570 & 67.7 & 38.5 & 63.4 & 3.72 & 151 & 191 \\
\hline & $4 / 27 / 15$ & 16.1 & 883 & 7.6 & 0.1 & 568 & 70.5 & 39.1 & 64.3 & 3.9 & 184 & 182 \\
\hline & $3 / 22 / 16$ & 16.0 & 897 & 7.4 & 0.0 & 561 & 77.8 & 41.6 & 74.6 & 4.1 & 193 & 190 \\
\hline \multirow[t]{4}{*}{ WD 11} & $5 / 3 / 04$ & 18.4 & 677 & 7.7 & 21.5 & 440 & 69.0 & 31.6 & 68.1 & 1.7 & 187 & 89.7 \\
\hline & $3 / 8 / 11$ & 16.0 & 891 & 7.7 & 9.9 & 533 & 75.2 & 36.6 & 51.2 & 2.15 & 186 & 179 \\
\hline & $2 / 7 / 12$ & 15.4 & 798 & 7.4 & 9.5 & 529 & 74.6 & 34.5 & 51.6 & 2.05 & 193 & 169 \\
\hline & $4 / 15 / 13$ & 16.1 & 832 & 6.7 & 11.4 & 519 & 71.5 & 34.3 & 53.9 & 2.14 & 194 & 166 \\
\hline \multirow[t]{4}{*}{ (replicate) } & $4 / 15 / 13$ & 16.1 & 832 & 6.7 & 11.4 & 527 & 73.9 & 34.5 & 55 & 2.08 & 194 & 167 \\
\hline & $4 / 28 / 14$ & 16.2 & 818 & 7.3 & 3.1 & 520 & 75.1 & 32.7 & 55.3 & 1.98 & 154 & 162 \\
\hline & $4 / 27 / 15$ & 16.2 & 807 & 7.8 & 4.5 & 513 & 74.4 & 32.6 & 54.9 & 2.1 & 193 & 149 \\
\hline & $3 / 21 / 16$ & 16.3 & 826 & 7.6 & 3.9 & 524 & 80.5 & 35.7 & 61.5 & 2.2 & 202 & 156 \\
\hline WD 12 & $9 / 12 / 02$ & - & 335 & 7.9 & - & 202 & 37 & 13 & 9.0 & 1.6 & 115 & 19 \\
\hline (') & $3 / 9 / 11$ & 19.9 & 1,670 & 7.1 & 13.4 & 1,150 & 132 & 57.3 & 150 & 3.0 & 116 & 440 \\
\hline (') & $2 / 8 / 12$ & 19.3 & 2,100 & 7.2 & 9.2 & 1,510 & 173 & 75.0 & 188 & 3.5 & 124 & 665 \\
\hline WD 14 & $3 / 24 / 16$ & 19.4 & 1,820 & 7.5 & 13.9 & 1,170 & 120 & 77.9 & 176 & 3.8 & 238 & 380 \\
\hline${ }^{1}$ WD 15 & 4/28/09 & 18.9 & 707 & 8.0 & 17.6 & 414 & 41.0 & 35.9 & 48.0 & 2.1 & 191 & 71.4 \\
\hline (') & $11 / 23 / 09$ & 18.8 & 729 & 8.3 & 14.5 & 436 & 43.3 & 33.6 & 57.5 & 2.1 & 184 & 80.4 \\
\hline$(1)$ & $3 / 16 / 10$ & 19.1 & 734 & 7.9 & 11.5 & 458 & 42.0 & 33.8 & 51.6 & 2.1 & 188 & 84.7 \\
\hline (') & $3 / 8 / 11$ & 19.1 & 816 & 8.0 & 12.5 & 469 & 45.9 & 39.3 & 60.6 & 2.1 & 182 & 91.3 \\
\hline \multirow[t]{3}{*}{$\left({ }^{1}\right)$} & $2 / 7 / 12$ & 19.1 & 821 & 8.0 & 18.8 & 473 & 42.2 & 38.2 & 67.8 & 2.1 & 169 & 102 \\
\hline & $4 / 17 / 13$ & 18.9 & 895 & - & 17.8 & 531 & 50.2 & 36.7 & 82.1 & 2.3 & 170 & 122 \\
\hline & $4 / 29 / 14$ & 18.8 & 969 & 7.7 & 11.7 & 583 & 52.4 & 38.0 & 88.4 & 2.3 & 169 & 132 \\
\hline \multirow[t]{3}{*}{ (replicate) } & $4 / 29 / 14$ & 18.8 & 969 & 7.7 & 11.7 & 560 & 52.2 & 38.0 & 88.5 & 2.34 & 167 & 128 \\
\hline & $4 / 29 / 15$ & 18.8 & 1,090 & 8.0 & 19.1 & 597 & 56.8 & 40.7 & 96.1 & 2.5 & 168 & 136 \\
\hline & $3 / 23 / 16$ & 19.0 & 1,130 & 8.0 & 18.9 & 665 & 62.6 & 43.1 & 107 & 2.6 & 171 & 151 \\
\hline \multirow[t]{6}{*}{ WD 16} & 4/27/09 & 18.7 & 444 & 7.7 & 8.7 & 255 & 44.1 & 23.0 & 13.2 & 1.9 & 136 & 33.6 \\
\hline & $11 / 24 / 09$ & 18.7 & 449 & 7.7 & 7.1 & 260 & 42.3 & 21.9 & 13.7 & 1.7 & 129 & 33.8 \\
\hline & $3 / 16 / 10$ & 18.7 & 441 & 7.6 & 5.1 & 262 & 41.7 & 22.4 & 12.3 & 1.8 & 135 & 33.0 \\
\hline & $3 / 8 / 11$ & 18.5 & 478 & 7.7 & 4.1 & 241 & 45.4 & 23.0 & 13.6 & 1.9 & 135 & 32.0 \\
\hline & $4 / 17 / 13$ & 18.7 & 432 & - & 8.1 & 269 & 43.1 & 22.7 & 13.4 & 1.76 & 137 & 34.4 \\
\hline & $4 / 29 / 14$ & 18.7 & 446 & 7.4 & 3.7 & 253 & 42.5 & 22.2 & 14.1 & 1.81 & 132 & 34.3 \\
\hline
\end{tabular}


Table 3. Field measurements and concentrations of major ions, selected trace elements, and nutrients in groundwater and surface water from selected sites in Sand Hollow, Washington County, Utah.-Continued

$\left[{ }^{\circ} \mathrm{C}\right.$, degrees Celsius; $\mu \mathrm{S} / \mathrm{cm}$, microsiemens per centimeter at 25 degrees Celsius; mg/L, milligrams per liter; $\mathrm{Cl} / \mathrm{Br}$, chloride to bromide ratio; $\mu \mathrm{g} / \mathrm{L}$, micrograms per liter; $\mu \mathrm{g} / \mathrm{L}$, micrograms per liter; —, no data available; <, less than; E, estimated; ft, feet]

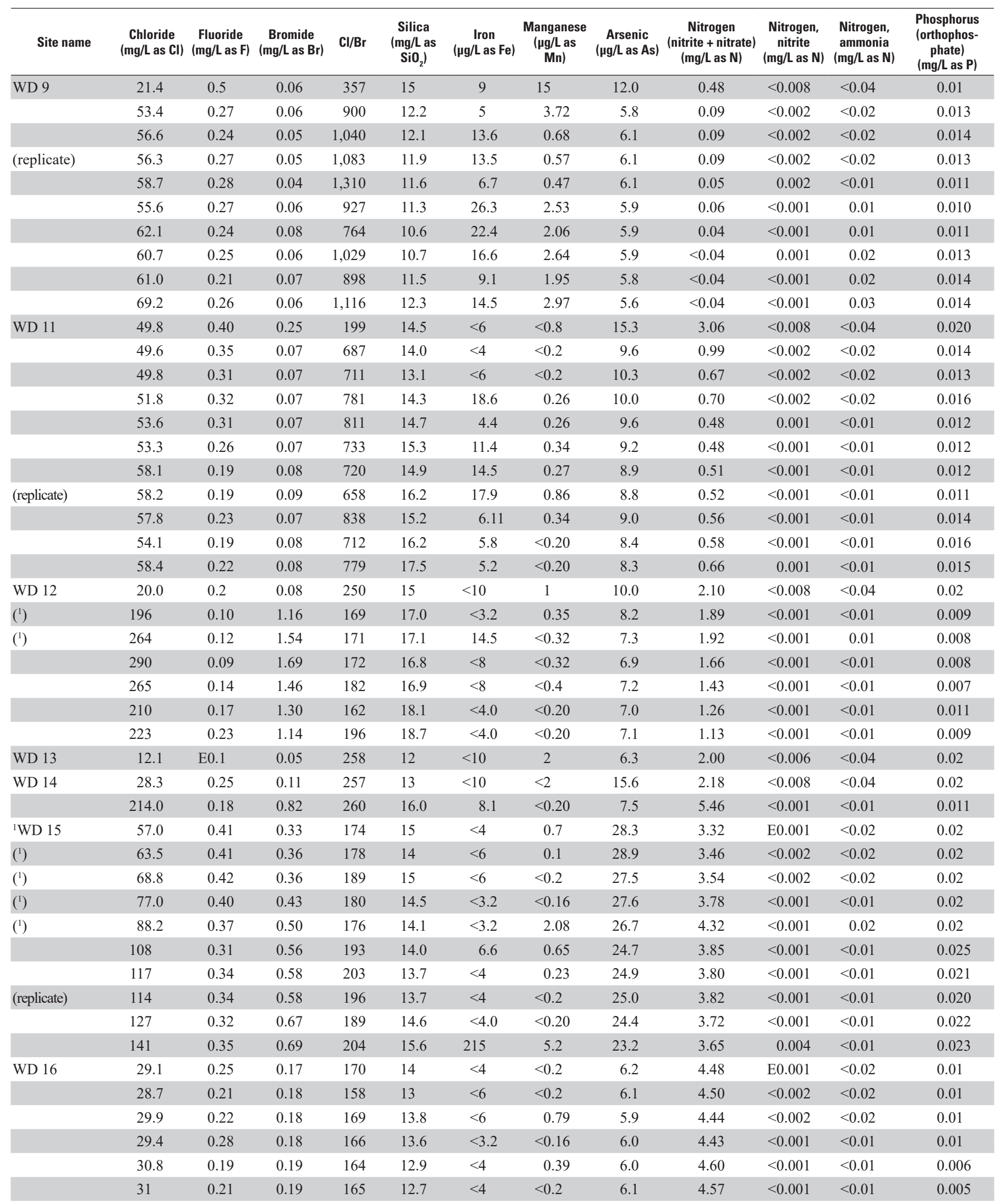


Table 3. Field measurements and concentrations of major ions, selected trace elements, and nutrients in groundwater and surface water from selected sites in Sand Hollow, Washington County, Utah.-Continued

$\left[{ }^{\circ} \mathrm{C}\right.$, degrees Celsius; $\mu \mathrm{S} / \mathrm{cm}$, microsiemens per centimeter at 25 degrees Celsius; $\mathrm{mg} / \mathrm{L}$, milligrams per liter; $\mathrm{Cl} / \mathrm{Br}$, chloride to bromide ratio; $\mu \mathrm{g} / \mathrm{L}$, micrograms per liter; $\mu \mathrm{g} / \mathrm{L}$, micrograms per liter; - , no data available; <, less than; E, estimated; ft, feet]

\begin{tabular}{|c|c|c|c|c|c|c|c|c|c|c|c|c|}
\hline Site name & Date & $\begin{array}{l}\text { Temper- } \\
\text { ature } \\
\left({ }^{\circ} \mathrm{C}\right)\end{array}$ & $\begin{array}{c}\text { Specific } \\
\text { conductance } \\
(\mu \mathrm{S} / \mathrm{cm})\end{array}$ & $\begin{array}{c}\mathrm{pH} \\
\text { (standard } \\
\text { units) }\end{array}$ & $\begin{array}{c}\text { Dissolved } \\
\text { oxygen } \\
\text { (mg/L) }\end{array}$ & $\begin{array}{c}\text { Dissolved- } \\
\text { solids con- } \\
\text { centration } \\
(\mathrm{mg} / \mathrm{L})\end{array}$ & $\begin{array}{l}\text { Calcium } \\
\text { (mg/L as Ca) }\end{array}$ & $\begin{array}{l}\text { Magnesium } \\
\text { (mg/L as Mg) }\end{array}$ & $\begin{array}{c}\text { Sodium } \\
\text { (mg/L as } \mathrm{Na} \text { ) }\end{array}$ & $\begin{array}{l}\text { Potassium } \\
\text { (mg/L as K) }\end{array}$ & $\begin{array}{c}\text { Alkalinity as } \\
\mathrm{CaCO}_{3} \\
\text { (mg/L) }\end{array}$ & $\begin{array}{c}\text { Sulfate } \\
\left(\mathrm{mg} / \mathrm{L} \text { as } \mathrm{SO}_{4}\right)\end{array}$ \\
\hline WD 16 & $4 / 29 / 15$ & 19.0 & 448 & 7.6 & 7.2 & 253 & 43.8 & 22.6 & 14.1 & 1.8 & 134 & 35.2 \\
\hline \multirow[t]{2}{*}{ WD 17} & $4 / 16 / 13$ & 19.4 & 545 & 7.6 & 11.0 & 332 & 51.3 & 20.5 & 34.0 & 2.0 & 141 & 56.1 \\
\hline & $3 / 23 / 16$ & 19.5 & 503 & 7.3 & 12.5 & 314 & 54.2 & 20.5 & 32.6 & 2.1 & 139 & 52.2 \\
\hline \multirow{2}{*}{ WD 18} & $3 / 9 / 11$ & 19.2 & 476 & 7.3 & 7.8 & 293 & 46.0 & 19.2 & 23.8 & 1.7 & 138 & 38.0 \\
\hline & $3 / 23 / 16$ & 19.5 & 491 & 7.5 & 8.1 & 269 & 48.7 & 20.2 & 0.81 & 1.8 & 144 & 42.4 \\
\hline${ }^{1}$ WD 19 & $3 / 10 / 11$ & 19.1 & 2,030 & 7.5 & 9.8 & 1,120 & 120 & 41.6 & 216 & 2.8 & 210 & 282 \\
\hline$(1,2)$ & $2 / 7 / 12$ & 18.6 & 1,900 & 7.5 & 6.8 & 1,150 & 123 & 43.1 & 181 & 2.9 & 246 & 349 \\
\hline WD 20 & 4/29/09 & 19.7 & 331 & 7.5 & 6.7 & 188 & 30.2 & 17.4 & 11.7 & 2.1 & 120 & 20.8 \\
\hline WD 21 & $3 / 22 / 16$ & 19.1 & 422 & 7.9 & 6.5 & 252 & 38.1 & 15.6 & 1.1 & 2.1 & 124 & 35.6 \\
\hline \multirow[t]{2}{*}{ WD 22} & $9 / 18 / 15$ & 19.3 & 676 & 7.6 & 17.9 & 419 & 63.8 & 25.3 & 38.2 & 2.0 & 142 & 133 \\
\hline & $3 / 23 / 16$ & 19.1 & 726 & 7.4 & 18.9 & 455 & 75.8 & 28.6 & 42.9 & 2.1 & 157 & 144 \\
\hline Well 1 at $890 \mathrm{ft}$ & $5 / 6 / 03$ & - & 350 & 7.8 & - & 216 & 31 & 21 & 7.4 & 2.9 & 130 & 19 \\
\hline Well 2 at $400 \mathrm{ft}$ & $10 / 10 / 02$ & - & 365 & 8.0 & - & 208 & 30 & 21 & 9.0 & 2.1 & 129 & 20 \\
\hline Well 2 at $615 \mathrm{ft}$ & $10 / 10 / 02$ & - & 365 & 8.1 & - & 190 & 30 & 21 & 6.5 & 2.5 & 131 & 16 \\
\hline Well 2 at $750 \mathrm{ft}$ & $10 / 10 / 02$ & - & 370 & 8.1 & - & 196 & 30 & 22 & 6.8 & 2.7 & 134 & 18 \\
\hline \multirow[t]{2}{*}{ Well 4} & $8 / 29 / 01$ & 20.1 & 480 & 8.0 & - & - & 36 & 19 & 38 & 2.0 & 128 & 58 \\
\hline & $9 / 11 / 02$ & 19.1 & 495 & 8.1 & - & 297 & 36 & 19 & 35 & 2.0 & 124 & 56 \\
\hline Well 8 at $245 \mathrm{ft}$ & $10 / 8 / 02$ & 19.0 & 550 & 7.5 & - & 323 & 49 & 20 & 35 & 2.1 & 141 & 70 \\
\hline & 4/29/09 & 16.1 & 790 & 8.4 & 7.0 & 503 & 54.3 & 37.4 & 53.7 & 4.0 & 147 & 189 \\
\hline & $11 / 24 / 09$ & 11.3 & 797 & 8.5 & 9.5 & 502 & 40.9 & 39.8 & 62.9 & 4.3 & 108 & 212 \\
\hline & $3 / 16 / 10$ & 9.8 & 817 & 8.0 & 9.4 & 534 & 43.5 & 38.4 & 57.6 & 4.6 & 120 & 211 \\
\hline & $3 / 9 / 11$ & 8.6 & 827 & 8.1 & 10.7 & 534 & 60.2 & 39.2 & 62.2 & 4.5 & 142 & 212 \\
\hline & $2 / 6 / 12$ & 6.1 & 821 & 8.2 & 9.2 & 534 & 53.6 & 39.8 & 61.3 & 4.3 & 138 & 213 \\
\hline & $4 / 17 / 13$ & 13.2 & 874 & 7.8 & 8.6 & 562 & 56.5 & 42.7 & 66.0 & 4.89 & 149 & 223 \\
\hline & $4 / 29 / 14$ & 15.2 & 846 & 9.0 & 10.5 & 535 & 52.0 & 40.8 & 66.2 & 4.66 & 134 & 207 \\
\hline & $4 / 27 / 15$ & 16.5 & 844 & 8.1 & 9.8 & 557 & 59.2 & 39.7 & 67.3 & 4.8 & 150 & 199 \\
\hline & $3 / 23 / 16$ & 12.9 & 828 & 8.2 & 9.9 & 514 & 56.6 & 40.8 & 69.3 & 4.7 & 146 & 192 \\
\hline \multirow[t]{7}{*}{ SH 1-18 } & $4 / 29 / 09$ & 14.3 & 800 & 8.6 & 9.6 & 502 & 56.1 & 37.2 & 53.6 & 4.2 & 146 & 190 \\
\hline & 8/10/09 & 25.3 & 800 & 8.7 & 9.1 & 501 & 42.6 & 38.3 & 60.5 & 4.3 & 110 & - \\
\hline & $3 / 16 / 10$ & 9.6 & 819 & 8.0 & 9.6 & 525 & 45.9 & 40.8 & 58.6 & 4.7 & 124 & 211 \\
\hline & $3 / 9 / 11$ & 8.1 & 820 & 8.2 & 10.7 & 528 & 60.1 & 39.6 & 61.2 & 4.43 & 140 & 210 \\
\hline & $2 / 8 / 12$ & 5.9 & 801 & 8.0 & 10.7 & 526 & 54.2 & 39.5 & 59.6 & 4.28 & 140 & 214 \\
\hline & $4 / 18 / 13$ & 12.5 & 883 & 8.0 & 8.7 & 553 & 58.1 & 43.1 & 67.1 & 4.73 & 148 & 223 \\
\hline & $4 / 30 / 14$ & 14.1 & 835 & 8.2 & - & 531 & 51.7 & 40.6 & 65.8 & 4.38 & 136 & 200 \\
\hline
\end{tabular}

${ }^{1}$ High or increasing dissolved-solids concentrations but low $\mathrm{Cl} / \mathrm{Br}$ ratios indicate groundwater is affected by flushing of naturally occurring solutes in vadose zone prior to reservoir construction.

${ }^{2}$ Replicate sample not reported because submersible pump was re-installed for sample collection and dissolved-solids concentration differed by more than 10 percent. 
Table 3. Field measurements and concentrations of major ions, selected trace elements, and nutrients in groundwater and surface water from selected sites in Sand Hollow, Washington County, Utah.-Continued

$\left[{ }^{\circ} \mathrm{C}\right.$, degrees Celsius; $\mu \mathrm{S} / \mathrm{cm}$, microsiemens per centimeter at 25 degrees Celsius; $\mathrm{mg} / \mathrm{L}$, milligrams per liter; $\mathrm{Cl} / \mathrm{Br}$, chloride to bromide ratio; $\mu \mathrm{g} / \mathrm{L}$, micrograms per liter; $\mu \mathrm{g} / \mathrm{L}$, micrograms per liter; - , no data available; <, less than; E, estimated; ft, feet]

\begin{tabular}{|c|c|c|c|c|c|c|c|c|c|c|c|c|}
\hline Site name & $\begin{array}{l}\text { Chloride } \\
\text { (mg/L as Cl) }\end{array}$ & $\begin{array}{l}\text { Fluoride } \\
\text { (mg/L as F) }\end{array}$ & $\begin{array}{c}\text { Bromide } \\
\text { (mg/L as } \mathrm{Br})\end{array}$ & $\mathrm{Cl} / \mathrm{Br}$ & $\begin{array}{c}\text { Silica } \\
\text { (mg/L as } \\
\left.\mathrm{SiO}_{2}\right)\end{array}$ & $\begin{array}{c}\text { Iron } \\
(\mu \mathrm{g} / \mathrm{L} \text { as } \mathrm{Fe})\end{array}$ & $\begin{array}{c}\text { Manganese } \\
(\mu \mathrm{g} / \mathrm{L} \text { as } \\
\mathrm{Mn})\end{array}$ & $\begin{array}{c}\text { Arsenic } \\
(\mu \mathrm{g} / \mathrm{L} \text { as As) }\end{array}$ & $\begin{array}{c}\text { Nitrogen } \\
\text { (nitrite + nitrate) } \\
\text { (mg/L as N) }\end{array}$ & $\begin{array}{c}\text { Nitrogen, } \\
\text { nitrite } \\
\text { (mg/L as N) }\end{array}$ & $\begin{array}{c}\text { Nitrogen, } \\
\text { ammonia } \\
\text { (mg/L as N) }\end{array}$ & $\begin{array}{c}\text { Phosphorus } \\
\text { (orthophos- } \\
\text { phate) } \\
\text { (mg/L as P) }\end{array}$ \\
\hline WD 16 & 31.6 & 0.20 & 0.19 & 168 & 13.3 & $<4.0$ & $<0.2$ & 5.8 & 4.69 & $<0.001$ & $<0.01$ & 0.008 \\
\hline \multirow[t]{2}{*}{ WD 17} & 55.2 & 0.34 & 0.30 & 187 & 15.0 & 20.8 & 0.57 & 12.8 & 3.06 & $<0.001$ & $<0.01$ & 0.015 \\
\hline & 52.1 & 0.42 & 0.28 & 185 & 15.8 & 6.4 & $<0.20$ & 12.9 & 2.78 & $<0.001$ & $<0.01$ & 0.014 \\
\hline \multirow{2}{*}{ WD 18} & 35.1 & 0.36 & 0.22 & 163 & 15.3 & $<3.2$ & $<0.16$ & 10.2 & 3.12 & $<0.001$ & $<0.01$ & 0.01 \\
\hline & 38.0 & 0.35 & 0.21 & 178 & 15.4 & 10.8 & 0.42 & 10.1 & 3.09 & $<0.001$ & $<0.01$ & 0.014 \\
\hline${ }^{1}$ WD 19 & 252 & 0.37 & 1.17 & 215 & 17.1 & $<3.2$ & $<0.16$ & 9.9 & 8.75 & $<0.001$ & $<0.01$ & 0.02 \\
\hline$(1,2)$ & 192 & 0.34 & 0.89 & 216 & 17.5 & 5.2 & 0.16 & 9.3 & 9.38 & $<0.001$ & $<0.01$ & 0.03 \\
\hline \multirow[t]{4}{*}{ WD 20} & 16.4 & 0.28 & 0.09 & 178 & 14 & 53 & 1 & 7.7 & 2.41 & E0.001 & $<0.02$ & 0.02 \\
\hline & 27.8 & 0.37 & 0.15 & 189 & 16.3 & 545 & 25.4 & 3.4 & 2.17 & $<0.001$ & $<0.01$ & 0.013 \\
\hline & 51.6 & 0.38 & 0.12 & 426 & 15.6 & $<4.0$ & 12.8 & 13.3 & 0.79 & $<0.001$ & $<0.01$ & 0.033 \\
\hline & 51.4 & 0.39 & 0.11 & 451 & 15.8 & 4.9 & $<0.20$ & 13.7 & 0.71 & $<0.001$ & $<0.01$ & 0.030 \\
\hline Well 1 at $890 \mathrm{ft}$ & 16.9 & 1.08 & - & - & 11 & 11 & 19 & 9.1 & 3.37 & 0.008 & 0.03 & 0.01 \\
\hline Well 2 at $400 \mathrm{ft}$ & 17.8 & 0.2 & - & - & 11 & 10 & 12 & 2.6 & 3.41 & 0.008 & 0.10 & 0.02 \\
\hline Well 2 at $615 \mathrm{ft}$ & 13.2 & 0.23 & - & - & 11 & 27 & 6 & 4.6 & 3.73 & 0.004 & $<0.04$ & 0.02 \\
\hline Well 2 at $750 \mathrm{ft}$ & 14.3 & 0.23 & 0.10 & 143 & 12 & 19 & 3 & 5.9 & 3.84 & $<0.008$ & 0.03 & 0.02 \\
\hline \multirow[t]{2}{*}{ Well 4} & 44.4 & E0.1 & 0.20 & 218 & 13 & $<10$ & $<3$ & 7.1 & 1.50 & $<0.006$ & $<0.04$ & 0.02 \\
\hline & 42.0 & 0.2 & 0.17 & 247 & 13 & $<10$ & $<2$ & 8.0 & 2.10 & $<0.008$ & $<0.04$ & 0.02 \\
\hline Well 8 at $245 \mathrm{ft}$ & 38.7 & 0.29 & 0.15 & 258 & 14 & $<10$ & 5 & 16.6 & 1.72 & 0.03 & 0.18 & 0.01 \\
\hline \multirow{9}{*}{ Boat ramp } & 54.9 & 0.31 & 0.04 & 1,227 & 2.9 & $<4$ & 0.3 & 1.4 & 0.04 & 0.002 & $<0.02$ & 0.008 \\
\hline & 60.4 & 0.28 & 0.05 & 1,313 & 1.5 & $<6$ & 0.2 & 1.6 & $<0.04$ & $<0.002$ & $<0.02$ & 0.008 \\
\hline & 61.7 & 0.30 & 0.04 & 1,374 & 1.4 & 6.3 & 1.7 & 1.3 & E0.03 & $<0.002$ & 0.02 & $<0.008$ \\
\hline & 57.0 & 0.30 & 0.04 & 1,390 & 4.0 & $<3.2$ & 2.0 & 1.13 & 0.10 & 0.004 & 0.03 & $<0.004$ \\
\hline & 58.3 & 0.27 & 0.05 & 1,108 & 4.2 & $<3.2$ & 0.86 & 1.01 & 0.12 & 0.001 & 0.01 & $<0.004$ \\
\hline & 65.8 & 0.25 & 0.08 & 875 & 2.9 & 5.2 & 1.22 & $<0.04$ & 0.09 & 0.007 & 0.04 & $<0.004$ \\
\hline & 65.3 & 0.27 & 0.05 & 1,280 & 1.2 & $<4$ & 0.74 & 1.3 & 0.04 & 0.002 & $<0.01$ & $<0.004$ \\
\hline & 67.3 & 0.24 & $<0.06$ & 1,346 & 4.0 & $<4.0$ & 1.10 & 1.5 & $<0.04$ & 0.002 & 0.02 & $<0.004$ \\
\hline & 67.2 & 0.26 & 0.046 & 1,461 & 4.3 & $<4.0$ & 0.73 & 1.6 & 0.11 & 0.002 & 0.02 & $<0.004$ \\
\hline \multirow[t]{7}{*}{ SH 1-18 } & 54.6 & 0.27 & 0.04 & 1,318 & 3.0 & $<4$ & 0.4 & 1.4 & 0.04 & 0.003 & 0.13 & 0.008 \\
\hline & - & 0.24 & - & - & 1.3 & 3 & 0.3 & 1.6 & $<0.04$ & $<0.002$ & $<0.02$ & 0.008 \\
\hline & 61.6 & 0.30 & 0.04 & 1,417 & 1.2 & 6.2 & 1.8 & 1.4 & E0.02 & $<0.002$ & 0.02 & $<0.008$ \\
\hline & 57.2 & 0.30 & 0.04 & 1,546 & 3.9 & $<3.2$ & 2.10 & 1.12 & 0.10 & 0.005 & 0.02 & $<0.004$ \\
\hline & 56.5 & 0.27 & 0.05 & 1,060 & 4.1 & $<3.2$ & 1.31 & 1.04 & 0.09 & 0.001 & 0.02 & $<0.004$ \\
\hline & 65.3 & 0.25 & 0.07 & 898 & 2.8 & 2.1 & 0.82 & 1.2 & 0.09 & 0.007 & 0.03 & $<0.004$ \\
\hline & 63.2 & 0.26 & 0.05 & 1,170 & 1.1 & $<4$ & 0.45 & 1.2 & 0.04 & 0.001 & $<0.01$ & $<0.004$ \\
\hline
\end{tabular}




\section{Summary}

Since its inception in 2002, diversions to Sand Hollow Reservoir from the nearby Virgin River generally have resulted in rising reservoir stage, ranging from about 3,000 feet in 2002 to a maximum of about 3,060 feet in 2006, which then fluctuated between about 3,040 and 3,060 feet from 2008 through 2016. Similarly, groundwater levels in monitoring wells closest to the reservoir generally rose between 2002 and 2006, and then fluctuated with reservoir altitude and pumpage rate from nearby production wells. Water levels in monitoring wells farther from the reservoir were still rising through 2016.

Between 2004 and 2016, about 37,000 acre-feet (acre-ft) of groundwater were withdrawn from production wells located near Sand Hollow Reservoir. French drains, installed to capture shallow seepage near the reservoir, were also pumped as they filled with water. About 9,000 acre-ft of groundwater were pumped from the North Dam drain between 2003 and 2016. Initially, this water was returned to the reservoir, but since 2007, has been used by Sand Hollow Resort for irrigation. About 2,300 acre-ft of water were pumped from the West Dam drain back into the reservoir from 2005 through 2016. In 2006, the West Dam Spring drain was constructed and has largely replaced the function of the West Dam drain. About 26,000 acre-ft were pumped from this drain into the Washington County Water Conservancy District's municipal supply system from 2006 through 2016.

Total annual surface-water inflow to Sand Hollow Reservoir ranged from about 56,000 acre-ft in 2005 to 800 acre-ft in 2007. Total inflow from 2002 through 2016 was about 256,000 acre-ft. The general increase in reservoir water-level altitude and surface area from 2002 to 2007 resulted in a steady increase in the volume of annual evaporation from about 1,000 to about 6,600 acre-ft through 2006, which then leveled off from 2007 through 2016. Total estimated cumulative evaporative loss from 2002 through 2016 was about 85,000 acre-ft. During this same period, annual reservoir recharge to the underlying Navajo Sandstone aquifer fluctuated between about 5,000 and 18,000 acre-ft. Total calculated reservoir recharge from 2002 through 2016 was about 141,000 acre-ft with a two standard deviation uncertainty of 13,300 acre-ft. From 2002 through 2016, calculated monthly recharge volumes ranged from 12 to almost 3,500 acre-ft, and average daily recharge rates (calculated for each month) ranged from 0.000 to 0.430 foot.

Annual water-quality sampling for field parameters, chemistry, and environmental tracers (tritium, chlorofluorocarbons, sulfur hexafluoride) was continued during 2015 and 2016. The most significant changes in water quality occurred at monitoring wells WD 4 (2,600 feet from the reservoir) and WD 12 (1,000 feet away), where increases in salinity (specific conductance) and dissolved oxygen (and environmental tracer concentrations at WD 4) indicate rising groundwater levels and mobilization of vadose-zone salts, likely a precursor to the arrival of reservoir recharge. At wells WD 9 (55 feet from the reservoir) and WD 11 (160 feet away), field parameters and water-quality and environmental tracer data indicate that reservoir recharge arrived several years prior to 2014 . At well WD 6 (1,000 feet away), increases in salinity and chloride/bromide ratios, along with environmental tracer data all indicate the recent arrival of reservoir recharge. Although well WD 8 is located only 500 feet from the reservoir, it is downgradient of an area of high natural recharge on Sand Mountain and may lie along a groundwater boundary between the two recharge mounds; relatively high concentrations of modern environmental tracers may be caused by natural recharge. At wells WD 15 and WD 16 (nested wells located 2,400 feet from the reservoir), water-quality and environmental tracer data indicate a rising water table, but no arrival yet of reservoir recharge.

\section{References Cited}

Heilweil, V.M., and Marston, T.M., 2011, Assessment of managed aquifer recharge from Sand Hollow Reservoir, Washington County, updated to conditions in 2010: U.S. Geological Survey Scientific Investigations Report 2011-5142, 39 p.

Heilweil, V.M., and McKinney, T.S., 2007, Net-infiltration map of the Navajo Sandstone outcrop area in western Washington County, Utah: U.S. Geological Survey Scientific Investigations Map 2988.

Heilweil, V.M., McKinney, T.S., Zhdanov, M.S., and Watt, D.E., 2007, Controls on the variability of net infiltration to desert sandstone: Water Resources Research, v. 43, W07431, DOI:10.1029/2006WR005113, 15 p.

Heilweil, V.M., Ortiz, G., and Susong, D.D., 2009, Assessment of managed aquifer recharge at Sand Hollow Reservoir, Washington County, Utah, updated to conditions through 2007: U.S. Geological Survey Scientific Investigations Report 2009-5050, 19 p.

Heilweil, V.M., and Solomon, D.K., 2004, Millimeter- to kilometer-scale variations in vadose-zone bedrock solutesImplications for estimating recharge in arid settings, in Phillips, F., Scanlon, B., and Hogan, J., eds., Groundwater recharge in a desert environment - The southwestern United States: Water Science and Application, v. 9, Washington, D.C., American Geophysical Union, p. 49-67.

Heilweil, V.M., Solomon, D.K., and Gardner, P.M., 2006, Borehole environmental tracers for evaluating net infiltration and recharge through desert bedrock: Vadose Zone Journal, v. 5, p. 98-120.

Heilweil, V.M., Solomon, D.K., and Ortiz, G., 2009, Silt and gas accumulation beneath an artificial recharge spreading basin, southwestern Utah, U.S.A: Boletin Geologico y Minero, v. 120, no. 2, p. 185-195. 
Heilweil, V.M., Solomon, D.K., Perkins, K.S., and Ellett, K.M., 2004, Gas-partitioning tracer test to quantify trapped gas during recharge: Ground Water, v. 42, no. 4, p. 589-600.

Heilweil, V.M., and Susong, D.D., 2007, Assessment of artificial recharge at Sand Hollow Reservoir, Washington County, Utah, updated to conditions through 2006:

U.S. Geological Survey Scientific Investigations

Report 2007-5023, 14 p.

Heilweil, V.M., Susong, D.D., Gardner, P.M., and Watt, D.E, 2005, Pre- and post-reservoir ground-water conditions and assessment of artificial recharge at Sand Hollow, Washington County, Utah, 1995-2005: U.S. Geological Survey Scientific Investigations Report 2005-5185, 74 p.

Heilweil, V.M., and Watt, D.E., 2011, Trench infiltration for managed aquifer recharge to permeable bedrock: Hydrological Processes 25, DOI: 10.1002/hyp.7833, p. $141-151$.

Marston, T.M., and Heilweil, V.M., 2012, Numerical simulation of groundwater movement and managed aquifer recharge from Sand Hollow Reservoir, Hurricane Bench area, Washington County, Utah: U.S. Geological Survey Scientific Investigations Report 2012-5236, 34 p.

Marston, T.M., and Heilweil, V.M., 2013, Assessment of managed aquifer recharge at Sand Hollow Reservoir, Washington County, Utah, updated to conditions in 2012: U.S. Geological Survey Scientific Investigations Report 2013-5057, 40 p.

Marston, T.M., and Heilweil, V.M., 2016, Assessment of managed aquifer recharge at Sand Hollow Reservoir, Washington County, Utah, updated to conditions through 2014: U.S. Geological Survey Open-File Report 20161078, $35 \mathrm{p}$.

McGuinness, J.L., and Bordne, E.F., 1971, A comparison of lysimeter-derived potential evapotranspiration with computed values: U.S. Department of Agriculture Technical Bulletin 1472, Washington, D.C., Agricultural Research Service, $71 \mathrm{p}$.

Rosenberry, D.O., Winter, T.C., Buso, D.C., and Likens, G.E., 2007, Comparison of 15 evaporation methods applied to a small mountain lake in the northeastern USA: Journal of Hydrology, v. 340, p. 149-166.

Wilde, F.D., and Radtke, D.B., 1998, National field manual for the collection of water-quality data-Field measurements: U.S. Geological Survey Techniques of Water-Resources Investigations, book 9, chap. A6, 233 p. 
For additional information, contact:

Director, Utah Water Science Center U.S. Geological Survey 2329 West Orton Circle

Salt Lake City, UT 84119-2047

801 908-5000

https://ut.water.usgs.gov/ 
